\title{
Exact four-point function and OPE for an interacting quantum field theory with space/time anisotropic scale invariance
}

\author{
Hidehiko Shimada $^{a}$ and Hirohiko Shimada ${ }^{b, c}$ \\ ${ }^{a}$ Yukawa Institute for Theoretical Physics, Kyoto University, \\ Kitashirakawa Oiwakecho, Sakyo-ku, Kyoto 606-8502 Japan \\ ${ }^{b}$ National Institute of Technology, Tsuyama College, \\ 624-1, Numa, Tsuyama, Okayama 708-8509 Japan \\ ${ }^{c}$ Mathematical and Theoretical Physics Unit, OIST, Graduate University, \\ Onna, Okinawa, 904-0495, Japan \\ E-mail: shimada.hidehiko@gmail.com, hirohikoshimada@gmail.com
}

Abstract: We identify a nontrivial yet tractable quantum field theory model with space/time anisotropic scale invariance, for which one can exactly compute certain fourpoint correlation functions and their decompositions via the operator-product expansion(OPE). The model is the Calogero model, non-relativistic particles interacting with a pair potential $\frac{g}{|x-y|^{2}}$ in one dimension, considered as a quantum field theory in one space and one time dimension via the second quantisation. This model has the anisotropic scale symmetry with the anisotropy exponent $z=2$. The symmetry is also enhanced to the Schrödinger symmetry. The model has one coupling constant $g$ and thus provides an example of a fixed line in the renormalisation group flow of anisotropic theories.

We exactly compute a nontrivial four-point function of the fundamental fields of the theory. We decompose the four-point function via OPE in two different ways, thereby explicitly verifying the associativity of OPE for the first time for an interacting quantum field theory with anisotropic scale invariance. From the decompositions, one can read off the OPE coefficients and the scaling dimensions of the operators appearing in the intermediate channels. One of the decompositions is given by a convergent series, and only one primary operator and its descendants appear in the OPE. The scaling dimension of the primary operator we computed depends on the coupling constant. The dimension correctly reproduces the value expected from the well-known spectrum of the Calogero model combined with the so-called state-operator map which is valid for theories with 
the Schrödinger symmetry. The other decomposition is given by an asymptotic series. The asymptotic series comes with exponentially small correction terms, which also have a natural interpretation in terms of OPE.

Keywords: Field Theories in Lower Dimensions, Integrable Field Theories, Renormalization Group, Space-Time Symmetries

ArXiv EPrint: 2107.07770 


\section{Contents}

1 Introduction 1

$\begin{array}{llc}2 & \text { The model } & 6\end{array}$

3 Four-point function $\quad \mathbf{8}$

3.1 Pairwise equal-time four-point function 8

$\begin{array}{lll}3.2 & \text { Double integral formula for the general four-point function } & 10\end{array}$

3.3 Four-point function in general position via generalised hypergeometric function 11

4 OPE decomposition of the four-point function

$\begin{array}{ll}\text { 4.1 Decomposition of pairwise equal-time four-point function } & 13\end{array}$

$\begin{array}{ll}4.1 .1 & 13\end{array}$

4.1.2 "t-channel" decomposition 16

4.1.3 The exponentially small corrections and "u-channel" contributions 20

4.2 Detailed analysis of "s-channel" decomposition 23

4.2.1 The contribution from the descendants of $\bar{\Phi}, \Phi$

$\begin{array}{lll}\text { 4.2.2 Reproducing full four-point function from OPE } & 27\end{array}$

4.3 Three-point function $\langle\Psi \Psi \bar{\Phi}\rangle \quad 28$

4.3.1 Three-point functions with two operators at equal-time 28

$\begin{array}{lll}\text { 4.3.2 Three-point functions in general position } & 29\end{array}$

4.4 Two-point function of the charge-zero operators appearing in the "t-channel" $\begin{array}{ll}\text { decomposition } & 30\end{array}$

5 Conclusion and discussion $\quad 33$

$\begin{array}{lll}\text { A Schrödinger symmetry } & 37\end{array}$

$\begin{array}{ll}\text { B Propagator in } 1 / r^{2} \text { potential } & 38\end{array}$

C Details of the computation of the three-point function $\langle\Psi \Psi \bar{\Phi}\rangle \quad 40$

$\begin{array}{ll}\text { C.1 Evaluation of the integral representation } & 40\end{array}$

C.2 Relabelling and properties of $w \quad 40$

C.3 Comparison to the general form of three-point functions dictated by Schrödinger symmetry 41

C.4 Three-point function in terms of parabolic cylinder functions and confluent hypergeometric functions $\quad 42$

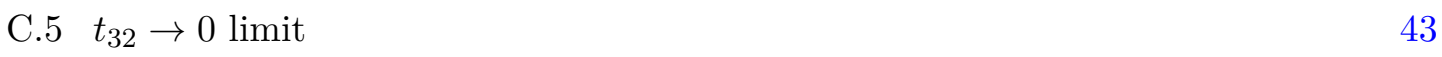

D Golkar and Son's analysis in Euclidean signature $\quad 44$

D.1 Preliminaries 45

D.2 OPE coefficients $\quad 45$ 
$\begin{array}{lll}\text { D.3 Boundary condition } & 47\end{array}$

D.4 Three-point function 48

E Free-Boson limit $\quad 49$

$\begin{array}{lll}\text { E.1 Pairwise equal-time four-point function } & 49\end{array}$

$\begin{array}{lll}\text { E.2 Three-point function } & 50\end{array}$

\section{Introduction}

The concept of the renormalisation group underlies the universality in various critical phenomena [1]. A quantum field theory with (isotropic) scale invariance is a fixed point of the renormalisation group flow in the space of quantum field theories and represents a universality class.

Quantum field theories invariant under space/time anisotropic scale transformation,

$$
\begin{aligned}
\vec{x} & \mapsto \alpha \vec{x}, \\
t & \mapsto \alpha^{z} t,
\end{aligned}
$$

are also of interest. The exponent $z \neq 1$ characterises the degree of anisotropy of the system. These theories are also fixed points of the renormalisation group flow in the generalised theory space of anisotropic quantum field theories. ${ }^{1}$

Because of this, these theories are also quite universal. There are many applications of quantum field theory models with anisotropic scale invariance. To illustrate the richness of the applications, let us list a few examples: dynamical critical phenomena in which time-dependent fluctuations around a critical point are considered [2, 3], quantum critical phenomena [4], more general non-equilibrium critical phenomena such as the directed percolation universality class [5-7] relevant for the onset of turbulence $[8,9]$, and the KPZ universality class in the surface growth phenomena [10]. Lucid introductions to these topics can be found in $[11,12]$. Another active area of research, with $z=2$, is the BEC/BCS crossover (also called the fermions at unitarity), systems of non-relativistic spin $1 / 2$ fermions with fine-tuned contact interaction, which can be experimentally realised in cold atom systems [13-17].

The operator-product expansion(OPE) [18, 19]

$$
\mathcal{O}_{i}(x) \mathcal{O}_{j}(0)=\sum_{k} C^{k}{ }_{i j}(x) \mathcal{O}_{k}(0)
$$

where $\mathcal{O}_{i}$ are local operators, summarises the short-distance physics of a quantum field theory, and is both useful and conceptually important. Consistency of successive OPEs imposes constraints on the theory, called the OPE associativity or the crossing symmetry. For the isotropic case, in particular, when the scale symmetry is enhanced into the

\footnotetext{
${ }^{1}$ In general, the renormalisation group flow connects two anisotropic theories characterised by different $z$.
} 
conformal symmetry [20], the constraints are often so powerful that consideration of them alone almost fixes the theory itself. This approach, originally conceived by Polyakov [21], is called the conformal bootstrap program. It had remarkable success for quantum field theories in two spacetime dimensions as pioneered by the fundamental work by Belavin, Polyakov and Zamolodchikov [22]. In recent years, starting with [23], it has become clear that the program can be successful also in higher spacetime dimensions. See e.g. [24, 25] for recent reviews.

It is natural to ask whether a similar bootstrap approach can be successful for anisotropic theories. Theories with $z=2$ would be the first target since in this case the scale symmetry can be extended to a larger symmetry, called the Schrödinger symmetry $[26,27]$. (The basic properties of the Schrödinger symmetry are briefly summarised in appendix A.) This enhancement is analogous to the enhancement of the scale invariance to the conformal symmetry which occurs for many interesting isotropic theories. ${ }^{2}$ In particular, if the Schrödinger symmetry is present, one can classify the local operators into primary operators and their descendants (those operators obtained by acting with spacetime derivatives on the primary operators), where the primary operators are defined by requiring that they commute with certain generators of the Schrödinger symmetry. ${ }^{3}$ For the isotropic case, the representation theory of the conformal symmetry, including the classification of operators into primary operators and their descendants, is a key tool in the conformal bootstrap program. The analogous representation theory of the Schrödinger symmetry relevant for the classification of operators can be found in [30,31] and references therein. Constraints on the correlation functions imposed by the Schrödinger symmetry, analogous but less restrictive compared to the isotropic case, are derived by Henkel [32, 33].

Somewhat surprisingly, the study of OPE for theories with anisotropic scale invariance started only relatively recently $[34,35]$. We expect the OPE for anisotropic theories to present new features since the short-distance behaviours of isotropic and anisotropic theories are markedly different. For example, the behaviour of the two-point function of scalar primary operators in $z=1$ conformal field theory (CFT) is

$$
\langle\mathcal{O}(x) \mathcal{O}(0)\rangle=\frac{1}{|x|^{2 \Delta}},
$$

whereas in $z=2$ Schrödinger invariant theory, it is

$$
\langle\mathcal{O}(t, \boldsymbol{x}) \overline{\mathcal{O}}(0, \mathbf{0})\rangle=\left\{\begin{array}{ll}
\frac{1}{t^{\Delta}} e^{-\frac{N_{\overline{\mathcal{O}}} \boldsymbol{x}^{2}}{2 t}} & (t>0) \\
0 & (t<0)
\end{array} .\right.
$$

\footnotetext{
${ }^{2}$ See [28] for a review of the criteria for symmetry enhancement in the isotropic case. The general criteria for the enhancement of $z=2$ scale invariance to the Schrödinger symmetry are not understood. Discussion of this issue for a class of models can be found in [28, 29].

${ }^{3}$ To be precise, a primary operator $\mathcal{O}(0, \mathbf{0})$ can be characterised by the conditions $[C, \mathcal{O}(0, \mathbf{0})]=0$ and $\left[K_{i}, \mathcal{O}(0, \mathbf{0})\right]=0$ in the notation explained in appendix A. We note that, in principle, one can define the concept of primary operators indirectly even if both the conformal and Schrödinger symmetries are absent (thus even if $z \neq 1$ and $z \neq 2$ ) by the condition that a primary operator can never be obtained as a spacetime derivative of other fields.
} 
Here, $\overline{\mathcal{O}}$ is the complex conjugate of the operator $\mathcal{O}$, and $N_{\overline{\mathcal{O}}}>0$ is the $\mathrm{U}(1)$ charge, which is contained in the Schrödinger symmetry, ${ }^{4}$ of the operator $\mathcal{O}$. Thus, the behaviour in the limit $t \rightarrow 0, \boldsymbol{x} \rightarrow 0$ in the anisotropic theory depends heavily on the precise manner of taking the limit and is more involved compared to the isotropic case.

Because of this difference, it is important to understand general questions regarding the OPE in the anisotropic theories such as "What are the convergence properties of the OPEs?" and "Does the operator associativity hold?".

For this purpose, it would be useful to have exactly solvable yet nontrivial examples of quantum field theory models. For the isotropic case, the two-dimensional Ising model and the massless Thirring model (which is equivalent to the compactified free-boson CFT via bosonisation) played an instrumental role when the ideas of OPE and the anomalous dimensions were established $[18,19,36-38]$. Exactly solvable models also gave substantial support to the development of two-dimensional conformal field theory [22]. We may hope that study of exactly solvable anisotropic models may play a similar role in the understanding of the $z \neq 1$ fixed points of the renormalisation group.

In this paper, we identify an interacting yet highly tractable model with anisotropic $z=2$ scale invariance and its extension to the Schrödinger symmetry. ${ }^{5}$ The model is the well-known Calogero model [39-43] considered as a quantum field theory in one space and one time dimension via the second quantisation.

We exactly compute the nontrivial four-point function of the fundamental fields of the theory, $\left\langle\Psi\left(t_{4}, x_{4}\right) \Psi\left(t_{3}, x_{3}\right) \bar{\Psi}\left(t_{2}, x_{2}\right) \bar{\Psi}\left(t_{1}, x_{1}\right)\right\rangle$. The result takes a particularly simple form when $t_{1}=t_{2}=0$ and $t_{3}=t_{4}=t$. It is expressed in terms of the modified Bessel function. We call these special four-point functions "pairwise equal-time". For the generic case, we give an expression of the four-point function in terms of a double convolution integral involving the pairwise equal-time four-point function and the propagator of non-relativistic free particles. The double convolution integral can also be evaluated using a generalised hypergeometric function.

We decompose the pairwise equal-time four-point function in two different ways via OPE, thereby explicitly verifying the associativity of the OPE for the first time for an interacting quantum field theory with anisotropic scale invariance. From the decomposition, one can read off the OPE coefficients and the scaling dimensions of the operators appearing in the intermediate channel.

One of the decompositions is obtained by expanding the pairwise equal-time four-point function by the parameter $\frac{x^{2}}{t}$, where $x$ refers collectively to $x_{21}=x_{2}-x_{1}, x_{43}=x_{4}-x_{3}$. This decomposition arises from the OPE of $\bar{\Psi}\left(0, x_{2}\right) \bar{\Psi}\left(0, x_{1}\right)$ and of $\Psi\left(t, x_{4}\right) \Psi\left(t, x_{3}\right)$. The decomposition can be schematically represented as

$$
\left\langle\Psi\left(t, x_{4}\right) \Psi\left(t, x_{3}\right) \bar{\Psi}\left(0, x_{2}\right) \bar{\Psi}\left(0, x_{1}\right)\right\rangle=\sum_{\bar{\Psi}_{1}} Y_{\bar{\Psi}_{2}}^{\Psi_{3}},
$$

\footnotetext{
${ }^{4}$ The $\mathrm{U}(1)$ charge is a central charge, i.e. it commutes with all other charges in the Schrödinger symmetry. In some literature, this $\mathrm{U}(1)$ charge is called the "mass" parameter.

${ }^{5}$ A quantum field theory possessing the Schrödinger symmetry is also called a "non-relativistic CFT" in recent literature.
} 
where the subscripts of $\Psi$ and $\bar{\Psi}$ are the labels of the spacetime points. We will call this expansion the "s-channel" decomposition of the four-point function. The expansion is convergent. Only one primary operator (together with its descendants) appears in the intermediate channel. Thus the four-point function is the analogue of the conformal block which plays an important role in the conformal bootstrap program. The primary operator has $\mathrm{U}(1)$ charge 2 . The scaling dimension of the primary operator depends on the coupling constant of the theory. The result is consistent with the well-known energy spectrum of the Calogero model, combined with the so-called state-operator map [44, 45], a relation between the scaling dimensions of the operators of a system with the Schrödinger symmetry and the energy spectrum of the theory put in an external harmonic oscillator potential.

The other decomposition is the expansion of the four-point function by $\frac{t}{x^{2}}$. This decomposition corresponds to the OPE of $\Psi\left(t, x_{3}\right) \bar{\Psi}\left(0, x_{1}\right)$ and of $\Psi\left(t, x_{4}\right) \bar{\Psi}\left(0, x_{2}\right)$ (where $x_{1}<x_{2}, x_{3}<x_{4}$ are assumed) and we call it the "t-channel" decomposition,

$$
\left.\left\langle\Psi\left(t, x_{4}\right) \Psi\left(t, x_{3}\right) \bar{\Psi}\left(0, x_{2}\right) \bar{\Psi}\left(0, x_{1}\right)\right\rangle=\sum_{\bar{\Psi}_{1}}^{\Psi_{3}}\right\rangle\left\langle_{\bar{\Psi}_{2}}^{\Psi_{4}}\right.
$$

We found that this decomposition is an asymptotic expansion.

The asymptotic nature may be understood intuitively as follows. As can be seen, for example, in (1.4), correlation functions in a Schrödinger invariant theory generically involve exponential factors of the form $e^{-a \frac{x^{2}}{t}}$, where $a$ is a numerical constant. These exponential factors play the role of the "instanton effect" if we think about $\frac{t}{x^{2}}$ as the "coupling constant". As is well-known, the asymptotic nature of a perturbation series is inherently related to the existence of the non-perturbative "instanton effect". ${ }^{6}$ (See, for example, [51].) Thus, one could have anticipated the asymptotic nature of the expansion in $\frac{t}{x^{2}}$ from the presence of the factors $e^{-a \frac{x^{2}}{t}}$ in Schrödinger invariant theories.

The operators appearing in the intermediate channel of the "t-channel" decomposition have vanishing $\mathrm{U}(1)$ charges. The charge-zero operators are important in particular because they include currents associated with any internal symmetry (including the $\mathrm{U}(1)$ symmetry in the Schrödinger symmetry) and the energy-momentum tensor. But they are elusive since the technique of the state-operator map is not applicable for them. The charge-zero sector is studied from the perspective of Schrödinger symmetry (and its infinite extension for specific models, the fermion at unitarity) in [52] and [53]. We study these charge-zero operators directly via the decomposition of the four-point function. For example, we will show that some charge-zero operators have non-vanishing two-point functions only if they are put on the same time slice.

\footnotetext{
${ }^{6}$ For the isotropic case, scale-invariant theories have convergent OPEs [46-48] whereas for general quantum field theories without scale invariance OPEs are asymptotic [49]. One explanation of this is as follows. (See the discussion below (2.11) of [50].) If the OPE (which is an expansion in terms of $x$ ) is asymptotic, it would imply the existence of the non-perturbative "instanton" effect of the form $e^{-\frac{l^{2}}{x^{2}}}$ where $l$ is a length scale. This is impossible for scale-invariant theories, hence OPEs cannot be asymptotic for these theories whereas theories without the scale invariance have asymptotic OPEs. Our intuitive understanding of the asymptotic nature of the "t-channel" decomposition is reminiscent of this explanation.
} 
The asymptotic expansion comes with exponentially small correction terms, which also can be interpreted naturally in terms of OPE: we found that the exponentially small terms are inherently related to the "u-channel" contributions arising from OPEs of $\Psi\left(t, x_{3}\right) \bar{\Psi}\left(0, x_{2}\right)$ and of $\Psi\left(t, x_{4}\right) \bar{\Psi}\left(0, x_{1}\right)$. These terms can be schematically represented as

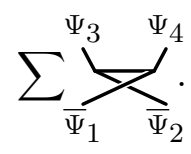

Some general properties of the OPE in the $z=2$ Schrödinger invariant theory have been uncovered in recent years [45, 52, 54]. Golkar and Son pointed out in [52], among other important results, that the restrictions imposed by the symmetry on the correlation functions become much stronger if one of the operators saturates the unitarity bound. Goldberger, Khandker and Prabhu proved the convergence of the OPE for the case when the operators in the intermediate channel have nonzero U(1) charges [45]. Pal studied Schrödinger invariant field theories focusing on the $\mathrm{SL}(2, \mathbb{R})$ subgroup of the Schrödinger symmetry and uncovered properties of correlation functions of operators which are aligned on a timelike line [54]. In particular, it was shown that the OPE relevant for these correlation functions converges even when the OPE involves charge-zero operators.

The results in this paper obtained for a particular solvable model confirm and supplement these general results. We compute the explicit OPE coefficients and show that the OPE converges for the "s-channel" OPE decomposition associated with charge-two operators in the intermediate channel. This is consistent with the results in [45]. The spacetime dependence of the three-point function we compute by pinching two insertions in the four-point function agrees with the result of [52] based on the Schrödinger symmetry.

On the other hand, we found novel features which presumably are shared by general Schrödinger invariant theories. The OPE decomposition associated with the "t-channel" OPE (involving charge-0 operators) is asymptotic, rather than convergent. This does not contradict the results of Pal [54]. We are studying different correlation functions: we consider the case where the operators are spatially separated, whereas in [54] the operators are separated only in the timelike direction.

The organisation of this paper is as follows. In section 2, we discuss the model and establish the notation. In section 3, we describe the computation of the four-point functions of fundamental fields in the model. Section 4 is devoted to what can be read off from the four-point function. We will decompose the four-point function via OPE in two ways (the "s-channel" and "t-channel" decompositions). We examine the detailed properties of these decompositions, including the identification of the unique primary operator (whose scaling dimension depends on the coupling constant) and the computation of the OPE coefficients in the "s-channel" decomposition. We discuss the asymptotic nature of the "t-channel" decomposition and the exponentially small corrections for the asymptotic series, which can be interpreted as the "u-channel" contributions. We also compute a three-point function by starting from the four-point function using OPE. Section 5 contains final comments. Several appendices give auxiliary results. 


\section{The model}

The Hamiltonian of the Calogero model (or the Calogero-Marchioro model) [39-43] in the first quantised formulation is ${ }^{7}$

$$
H=-\frac{1}{2} \sum_{i} \partial_{i}^{2}+\sum_{i<j} \frac{g}{\left(x_{j}-x_{i}\right)^{2}}
$$

We work in the convention where the mass of the particle is set to unity.

For $x_{j}-x_{i} \rightarrow 0$, the solution to the Schrödinger equation behaves as $\Psi \sim\left|x_{j}-x_{i}\right|^{\lambda}$ where $g=\lambda(\lambda-1)$. The coupling constant $g$ should satisfy $g \geq-\frac{1}{4}$ in order that the energy spectrum be bounded below [59, section 35]. Solutions with $0 \leq \lambda$ are considered as acceptable. In the regime $-\frac{1}{4}<g<0$, there are two solutions satisfying $\lambda>0$ for given $g$. Corresponding to these two possible boundary conditions, we have two different theories. ${ }^{8}$ Thus $\lambda \geq 0$ provides a good parametrisation of the interacting theory. For $\lambda=0$ and $\lambda=1$, the pair potential vanishes. These points are equivalent to the free bosons and free fermions (or equivalently, bosons interacting with the infinitely large repulsive $\delta$-function potential), respectively. It is also convenient (to conform with the convention used for the Bessel functions) to use another parameter $\nu$ defined by

$$
\begin{aligned}
& \nu=\lambda-\frac{1}{2}, \quad-\frac{1}{2} \leq \nu, \\
& g=\left(\nu-\frac{1}{2}\right)\left(\nu+\frac{1}{2}\right) .
\end{aligned}
$$

In the second quantised formulation, the action is

$$
S=\int d t d x\left(\bar{\Psi} \partial_{t} \Psi+\frac{1}{2}|\nabla \Psi|^{2}\right)+\frac{g}{2} \int d t d x d y|\Psi|^{2}(x) \frac{1}{(x-y)^{2}}|\Psi|^{2}(y) .
$$

We consider the Euclidean statistical field theory in this paper. The canonical (anti)commutation relations are,

$$
\begin{aligned}
{\left[\Psi(x), \bar{\Psi}\left(x^{\prime}\right)\right]_{ \pm} } & =\delta\left(x-x^{\prime}\right), \\
{[\Psi(x), \Psi(y)]_{ \pm} } & =0, \\
{[\bar{\Psi}(x), \bar{\Psi}(y)]_{ \pm} } & =0 .
\end{aligned}
$$

The signs here are chosen according to whether we consider the bosonic or the fermionic model.

We wish to note however that, as is well known, in the Calogero model, the difference between the bosonic and the fermionic theory is not important in the following sense. ${ }^{9}$ One

\footnotetext{
${ }^{7}$ The term "Calogero model" often refers to particles interacting via a pairwise potential of the form $V(r)=g / r^{2}+a r^{2}$, or equivalently, particles interacting via a pairwise potential $V(r)=g / r^{2}$ put in an external harmonic oscillator potential. The model we consider can also be considered as the infinite volume limit (with the total number of particles, not the density, fixed) of the Sutherland model [55-58], particles on a circle (with radius $R$ ) interacting with a pairwise potential of the form $V(r)=g /\left(R^{2} \sin ^{2} \frac{r}{R}\right)$.

${ }^{8}$ The possibility of considering the branch with the smaller value of $\lambda$ was discussed already in [55]. For a review of the Calogero and related models containing an explanation of this point, see [60].

${ }^{9}$ This was already pointed out in the original papers by Calogero [39, 40, 42, 43] and emphasised and explained in detail in [61]. See also the review article [60].
} 
can solve the Schrödinger equation of the model in the $n$-particle sector with the restriction $x_{1}<x_{2}<\cdots<x_{n}$, imposing the correct boundary condition $\Psi \sim\left(x_{i+1}-x_{i}\right)^{\lambda}$ when $x_{i+1}-x_{i} \rightarrow+0$. This is sufficient for the understanding of the properties of the Calogero model. Note that the boundary condition on $\Psi$ implies that there is no tunnelling amplitude of the particle (for $\lambda>0$ ), say, 1 from the region $x_{1}<x_{2}$ to the region $x_{1}>x_{2}$; the wave function vanishes at $x_{1}=x_{2}$. One can define the wave function for the regions where the condition $x_{1}<x_{2}<\cdots<x_{n}$ is not satisfied, by complete symmetrisation or antisymmetrisation for bosons or fermions, respectively. Whether one is dealing with bosons or fermions does not affect physical observables such as the energy levels (when an external harmonic oscillator potential is present) of the system. In our analysis, we also found that, for example, the four-point functions are the same for fermions and bosons, provided that the ordering of the particles are properly specified. We will work both for the bosonic and fermionic models throughout this paper, except when otherwise explicitly stated.

The Calogero model possesses the Schrödinger symmetry as first shown in [62]. Thus the model constitutes a fixed line of the renormalisation group parametrised by $\nu \geq-\frac{1}{2}$. The special significance of the potential energy $1 / r^{2}$ regarding scale invariance was noted also in $[63,64]$. The $\mathrm{U}(1)$ charge in the Schrödinger symmetry is given by

$$
N=\int \bar{\Psi} \Psi d x
$$

and coincides with the particle number.

Although the Lagrangian (2.4) is non-local, we will show that this theory has local OPEs. This is not too surprising; there are examples of quantum field theories with nonlocal interaction, which nonetheless exhibit critical properties described by a fixed point of the renormalisation group and can be studied by OPE and the conformal bootstrap such as systems with a non-local dipole-dipole interaction $[65,66]$ and Ising models with a non-local interaction term [67-70].

We will study the correlation functions of the model around the true vacuum, i.e. the state in which no particles are present. The simplest of such correlation functions is the two-point function of the fundamental fields,

$$
\langle\Psi(t, x) \bar{\Psi}(0,0)\rangle=\left\{\begin{array}{ll}
\sqrt{\frac{1}{2 \pi t}} e^{-\frac{(x-y)^{2}}{2 t}} & (t>0) \\
0 & (t<0)
\end{array} .\right.
$$

The U(1) charges of the fundamental fields are $N_{\Psi}=-1, N_{\bar{\Psi}}=+1$. The two-point function (2.9) is not renormalised, i.e. agrees with the free-theory result. In particular, the fields $\Psi, \bar{\Psi}$ have scaling dimension $\frac{1}{2}$. See (1.4). ${ }^{10}$ This non-renormalisation is a consequence of the fact that the two-point functions are associated only with one-particle states, and one-particle states by construction are not affected by the interaction term. (There are no amplitudes to create virtual particles starting from the one-particle states in the model. Also, there are no vacuum polarisation effects.) General correlation functions around the true vacuum are, of course, nontrivial and contain dynamical information of the model as we will see in later sections of this paper.

\footnotetext{
${ }^{10}$ We fix the normalisation of $\Psi, \bar{\Psi}$ by the canonical (anti-)commutation relation (2.5).
} 
Correlation functions around the true vacuum are different from the correlation functions around the "finite-density vacuum" (the ground state with a constant finite density of particles) of the Calogero model, ${ }^{11}$ which have been extensively studied. See, for example, [71] and references therein. The reason we study the correlation functions around the true vacuum in this paper is that we are interested in the $z=2$ scale-invariant correlation functions; the presence of the nonzero density breaks the $z=2$ scale invariance spontaneously.

\section{Four-point function}

In this section, we will compute the nontrivial four-point function of the fundamental fields

$$
\begin{aligned}
& \left\langle\Psi\left(t_{4}, x_{4}\right) \Psi\left(t_{3}, x_{3}\right) \bar{\Psi}\left(t_{2}, x_{2}\right) \bar{\Psi}\left(t_{1}, x_{1}\right)\right\rangle \\
& \quad=\left\langle 0\left|T \Psi\left(t_{4}, x_{4}\right) \Psi\left(t_{3}, x_{3}\right) \bar{\Psi}\left(t_{2}, x_{2}\right) \bar{\Psi}\left(t_{1}, x_{1}\right)\right| 0\right\rangle,
\end{aligned}
$$

of the model described in the previous section.

\subsection{Pairwise equal-time four-point function}

The four-point function can be easily computed for the special, pairwise equal-time case, i.e. when

$$
\begin{aligned}
& t_{1}=t_{2}=0, \\
& t_{3}=t_{4}=t>0 .
\end{aligned}
$$

If $t<0$ the four-point function trivially vanishes since the operator $\Psi(t, x)$ annihilates the vacuum. The key observation is that the pairwise equal-time correlation function is equivalent to the two-particle Feynman propagator $K^{(2)}\left(x_{3}, x_{4} ; x_{1}, x_{2} ; t\right)$ in the first quantised formulation, i.e. the transition amplitude of two particles starting at $x_{1}, x_{2}$ arriving at $x_{3}, x_{4}$ after time $t$ passes, ${ }^{12}$

$$
\begin{aligned}
\left\langle\Psi\left(t, x_{4}\right) \Psi\left(t, x_{3}\right) \bar{\Psi}\left(0, x_{2}\right) \bar{\Psi}\left(0, x_{1}\right)\right\rangle & =\left\langle 0\left|T \Psi\left(t, x_{4}\right) \Psi\left(t, x_{3}\right) \bar{\Psi}\left(0, x_{2}\right) \bar{\Psi}\left(0, x_{1}\right)\right| 0\right\rangle \\
& =\left\langle 0\left|\Psi\left(t, x_{4}\right) \Psi\left(t, x_{3}\right) \bar{\Psi}\left(0, x_{2}\right) \bar{\Psi}\left(0, x_{1}\right)\right| 0\right\rangle \\
& =K^{(2)}\left(x_{3}, x_{4} ; x_{1}, x_{2} ; t\right) .
\end{aligned}
$$

The propagator $K^{(2)}$ is a solution of the two-body Schrödinger equation,

$$
i \partial_{t} K^{(2)}=\left(-\frac{1}{2} \partial_{3}^{2}-\frac{1}{2} \partial_{4}^{2}+g \frac{1}{\left(x_{4}-x_{3}\right)^{2}}\right) K^{(2)},
$$

with the initial condition

$$
\lim _{t \rightarrow 0+} K^{(2)}=\delta\left(x_{3}-x_{1}\right) \delta\left(x_{4}-x_{2}\right),
$$

where we assume for simplicity $x_{1}<x_{2}, x_{3}<x_{4}$.

\footnotetext{
${ }^{11}$ Correlation functions around the "finite-density vacuum" of the Calogero model are also equivalent to the correlation functions of the Sutherland model in the thermodynamic limit, the large-volume limit with the density fixed.

${ }^{12}$ For the fermionic theory, it is useful to consider the four-point function as the $\operatorname{limit} \lim _{\epsilon_{1} \rightarrow 0, \epsilon_{2} \rightarrow 0}\langle\Psi(t+$ $\left.\left.\epsilon_{2}, x_{4}\right) \Psi\left(t-\epsilon_{2}, x_{3}\right) \bar{\Psi}\left(\epsilon_{1}, x_{2}\right) \bar{\Psi}\left(-\epsilon_{1}, x_{1}\right)\right\rangle$. The limit is well-defined and does not depend on the sign of $\epsilon_{1}, \epsilon_{2}$.
} 
The relation (3.4) follows from the basic feature of the second quantisation. (See, for example, sections 64 and 65 of [59].) Let us recall that the state,

$$
\bar{\Psi}\left(x^{\prime}\right) \bar{\Psi}\left(x^{\prime \prime}\right)|0\rangle,
$$

in the second quantised formulation, where we use $\bar{\Psi}(x)$ to denote the creation operator in the $x$-representation, is a two-particle state, specified by the wave function,

$$
\Psi\left(x_{1}, x_{2}\right)=\frac{1}{\sqrt{2}}\left(\delta\left(x_{1}-x^{\prime}\right) \delta\left(x_{2}-x^{\prime \prime}\right) \pm \delta\left(x_{1}-x^{\prime \prime}\right) \delta\left(x_{2}-x^{\prime}\right)\right)
$$

in the first quantised formulation. (The sign \pm above refers to the bosonic and the fermionic model, respectively.) The equivalence of the pairwise equal-time four-point function and the propagator (3.4) immediately follows.

By separating out the centre of mass motion, the computation of the two-particle propagator reduces to that of the propagator of a particle in an external potential of the form $1 / r^{2}$. Defining the relative position $r=x_{2}-x_{1} \equiv x_{21}$, the relevant Hamiltonian is

$$
H_{\text {rel }}=-\partial_{r}^{2}+\frac{\lambda(\lambda-1)}{r^{2}}
$$

We can focus on the region $r>0$. The propagator for this potential was first computed by Peak and Inomata [72],

$$
\left\langle r^{\prime}\left|e^{-H_{\text {rel }} t}\right| r\right\rangle=\sqrt{r r^{\prime}} \frac{1}{2 t} e^{-\frac{r^{2}+r^{\prime 2}}{4 t}} I_{\nu}\left(\frac{r r^{\prime}}{2 t}\right),
$$

where $\nu=\lambda-\frac{1}{2}$. The boundary condition is such that the wave function behaves as $r^{\lambda}$ at $r \rightarrow 0$. For completeness, we will present a derivation of this result in appendix B.

The centre of mass contribution to the four-point function is

$$
\sqrt{\frac{1}{\pi t}} e^{-\frac{x^{2}}{t}}
$$

where $X$ is the change of the centre of mass from the initial to the final state,

$$
X=\frac{x_{3}+x_{4}}{2}-\frac{x_{1}+x_{2}}{2}=\frac{x_{31}+x_{42}}{2}=\frac{x_{32}+x_{41}}{2} .
$$

Hence the full four-point function is ${ }^{13}$

$$
\begin{aligned}
& \left\langle\Psi\left(t, x_{4}\right) \Psi\left(t, x_{3}\right) \bar{\Psi}\left(0, x_{2}\right) \bar{\Psi}\left(0, x_{1}\right)\right\rangle \\
& \quad=e^{-\frac{x_{21}^{2}+x_{43}^{2}+\left(x_{3}+x_{4}-x_{1}-x_{2}\right)^{2}}{4 t}} \times \sqrt{\frac{x_{21} x_{43}}{4 \pi t^{3}}} I_{\nu}\left(\frac{x_{21} x_{43}}{2 t}\right) .
\end{aligned}
$$

Here $t>0$ is assumed; if $t<0$ the correlation function trivially vanishes. Also, the conditions

$$
\begin{aligned}
& x_{21}>0, \\
& x_{43}>0,
\end{aligned}
$$

\footnotetext{
${ }^{13}$ It is easy to check that putting $\nu=-\frac{1}{2}$ in (3.13) reproduces the four-point function of the free bosonic theory. See appendix E.
} 
are assumed, which come from the assumption that the relative position $r$ is positive. The expression (3.13) is valid for both the bosonic and fermionic cases under these conditions.

It is easy to obtain the four-point function for the generic case. The results for the bosonic and the fermionic theory differ by a sign factor. For the bosonic theory, we have

$$
\begin{aligned}
& \left\langle\Psi\left(t, x_{4}\right) \Psi\left(t, x_{3}\right) \bar{\Psi}\left(0, x_{2}\right) \bar{\Psi}\left(0, x_{1}\right)\right\rangle \\
& \quad=e^{-\frac{x_{21}^{2}+x_{43}^{2}+\left(x_{3}+x_{4}-x_{1}-x_{2}\right)^{2}}{4 t}} \times \sqrt{\frac{\left|x_{21} x_{43}\right|}{4 \pi t^{3}}} I_{\nu}\left(\frac{\left|x_{21} x_{43}\right|}{2 t}\right),
\end{aligned}
$$

and, for the fermionic theory, we have

$$
\begin{aligned}
& \left\langle\Psi\left(t, x_{4}\right) \Psi\left(t, x_{3}\right) \bar{\Psi}\left(0, x_{2}\right) \bar{\Psi}\left(0, x_{1}\right)\right\rangle \\
& \quad=\operatorname{sgn}\left(x_{43}\right) \operatorname{sgn}\left(x_{21}\right) e^{-\frac{x_{21}^{2}+x_{43}^{2}+\left(x_{3}+x_{4}-x_{1}-x_{2}\right)^{2}}{4 t}} \times \sqrt{\frac{\left|x_{21} x_{43}\right|}{4 \pi t^{3}}} I_{\nu}\left(\frac{\left|x_{21} x_{43}\right|}{2 t}\right),
\end{aligned}
$$

where $\operatorname{sgn}(x)=\frac{x}{|x|}$.

\subsection{Double integral formula for the general four-point function}

The four-point function in a generic position can be computed by a convolution integral of the free particle propagator and the pairwise equal-time correlation function computed in the previous subsection. We consider the four-point function,

$$
\left\langle\Psi\left(t_{4}, x_{4}\right) \Psi\left(t_{3}, x_{3}\right) \bar{\Psi}\left(t_{2}, x_{2}\right) \bar{\Psi}\left(t_{1}, x_{1}\right)\right\rangle,
$$

assuming $t_{1}<t_{2}<t_{3}<t_{4}$ without loss of generality. If $t_{1}<t_{3}<t_{2}<t_{4}$, for example, then the four-point function trivially factorises into a product of two-point functions.

The double integral formula is

$$
\begin{aligned}
& \left\langle\Psi\left(t_{4}, x_{4}\right) \Psi\left(t_{3}, x_{3}\right) \bar{\Psi}\left(t_{2}, x_{2}\right) \bar{\Psi}\left(t_{1}, x_{1}\right)\right\rangle \\
= & \int_{-\infty}^{+\infty} \int_{-\infty}^{+\infty} K\left(x_{4} ; x_{4}^{\prime} ; t_{4}-t_{3}\right) K^{(2)}\left(x_{3}, x_{4}^{\prime} ; x_{1}^{\prime}, x_{2} ; t_{3}-t_{2}\right) K\left(x_{1}^{\prime} ; x_{1} ; t_{2}-t_{1}\right) d x_{1}^{\prime} d x_{4}^{\prime},
\end{aligned}
$$

where $K(x ; y ; t)$ is the free one-particle propagator,

$$
K(x ; y ; t)=\frac{1}{\sqrt{2 \pi t}} e^{-\frac{(x-y)^{2}}{2 t}},
$$

and the pairwise four-point function or equivalently the two-particle propagator,

$$
K^{(2)}\left(x_{3}, x_{4} ; x_{1}, x_{2} ; t\right)=\left\langle\Psi\left(t, x_{4}\right) \Psi\left(t, x_{3}\right) \bar{\Psi}\left(0, x_{2}\right) \bar{\Psi}\left(0, x_{1}\right)\right\rangle,
$$

is given by (3.16) or (3.17) according to whether the theory is bosonic or fermionic. The formula (3.19) holds because in the intervals $t_{1}<t<t_{2}$ and $t_{3}<t<t_{4}$ there is only a single particle as shown in figure 1.

There is, of course, also a simpler integral formula to compute a four-point function where only $\bar{\Psi}$ 's are inserted on the same time slice as shown in figure 2 ,

$$
\begin{aligned}
& \left\langle\Psi\left(t_{4}, x_{4}\right) \Psi\left(t_{3}, x_{3}\right) \bar{\Psi}\left(0, x_{2}\right) \bar{\Psi}\left(0, x_{1}\right)\right\rangle \\
& \quad=\int_{-\infty}^{+\infty} K\left(x_{4} ; x_{4}^{\prime} ; t_{4}-t_{3}\right) K^{(2)}\left(x_{3}, x_{4}^{\prime} ; x_{1}, x_{2} ; t_{3}\right) d x_{4}^{\prime},
\end{aligned}
$$




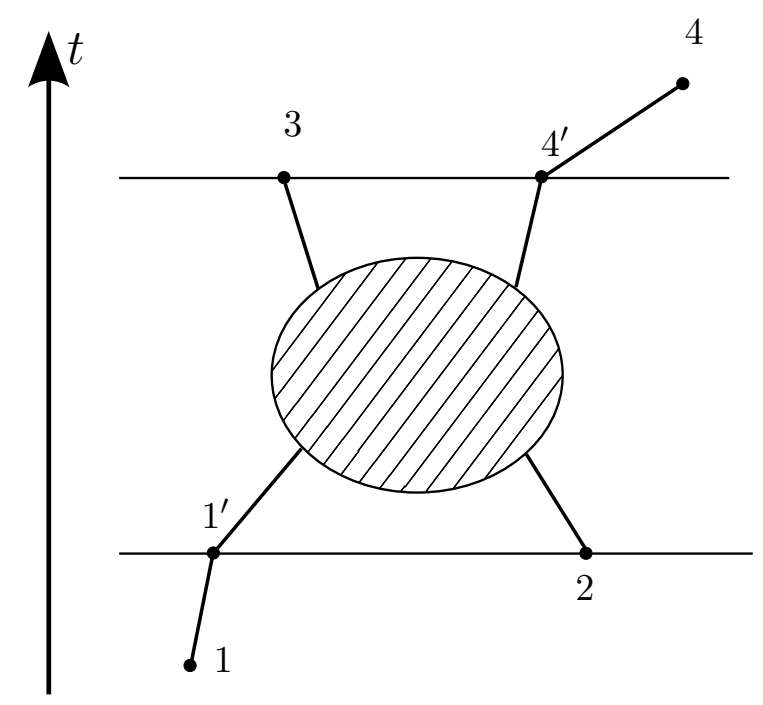

Figure 1. Horizontal lines are $t=t_{3}$ and $t=t_{2}$. The coordinates of the points $1^{\prime}$ and $4^{\prime}$ are $\left(t_{1}^{\prime}=t_{2}, x_{1}^{\prime}\right)$ and $\left(t_{4}^{\prime}=t_{3}, x_{4}^{\prime}\right)$ respectively. Both $x_{1}^{\prime}$ and $x_{4}^{\prime}$ are to be integrated from $-\infty$ to $+\infty$.

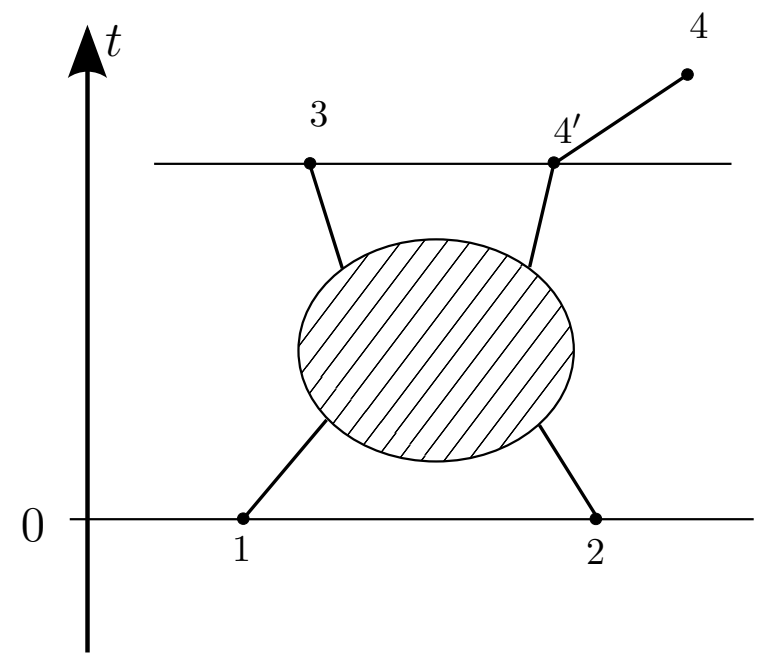

Figure 2. Horizontal lines are $t=t_{3}$ and $t=t_{1}=t_{2}=0$. The coordinates of the point $4^{\prime}$ are $\left(t_{4}^{\prime}=t_{3}, x_{4}^{\prime}\right) \cdot x_{4}^{\prime}$ is integrated from $-\infty$ to $+\infty$.

where $0=t_{1}=t_{2}<t_{3}<t_{4}$. This formula will be used later in section 4.3 .2 when we compute a three-point function.

\subsection{Four-point function in general position via generalised hypergeometric function}

The double integral (3.19) representing the four-point function

$$
\left\langle\Psi\left(t_{4}, x_{4}\right) \Psi\left(t_{3}, x_{3}\right) \bar{\Psi}\left(t_{2}, x_{2}\right) \bar{\Psi}\left(t_{1}, x_{1}\right)\right\rangle
$$

in general position can be expressed in terms of the generalised hypergeometric function. The detailed derivation of the formula and its consequences will be discussed in a separate 
publication. In this paper, we give the expression and discuss a few of its basic properties. We focus on the bosonic theory. We consider the nontrivial case, $t_{1}<t_{2}<t_{3}<t_{4}$.

The result is

$$
\begin{aligned}
& \left\langle\Psi\left(t_{4}, x_{4}\right) \Psi\left(t_{3}, x_{3}\right) \bar{\Psi}\left(t_{2}, x_{2}\right) \bar{\Psi}\left(t_{1}, x_{1}\right)\right\rangle \\
& \quad=\frac{e^{-\frac{x_{43}^{2}}{2 t_{43}}-\frac{x_{32}^{2}}{t_{32}}-\frac{x_{21}^{2}}{2 t_{21}}}}{(2 \pi)^{\frac{3}{2}} \sqrt{t_{43} t_{21}}} \frac{\Gamma^{2}\left(\frac{\nu}{2}+\frac{3}{4}\right)}{2^{\nu-1} \Gamma(\nu+1)} \tau^{\frac{\nu}{2}+\frac{3}{4}} \mathcal{F}\left(v_{123}, v_{234}, \tau\right),
\end{aligned}
$$

where the function $\mathcal{F}$ is a generalised hypergeometric function with three variables defined by the following triple series expansion,

$$
\mathcal{F}\left(v_{123}, v_{234}, \tau\right)=\sum_{\substack{p=0 \\ m, n=0}}^{\infty} \frac{(\tau)^{p}}{p !} \frac{\left(v_{123}\right)^{m}}{m !} \frac{\left(v_{234}\right)^{n}}{n !}\left[\Lambda_{p m n}^{(0)}+\sqrt{\tau v_{123} v_{234}} \Lambda_{p m n}^{(1)}\right]
$$

with the coefficients $\Lambda_{p m n}^{(j)}(j=0,1)$ given by

$$
\Lambda_{p m n}^{(j)}=\left(\nu+\frac{3}{2}\right)^{2 j} \frac{\left(\frac{\nu}{2}+\frac{3}{4}+j\right)_{p+m}\left(\frac{\nu}{2}+\frac{3}{4}+j\right)_{p+n}\left(\nu+\frac{1}{2}+j\right)_{2 p}}{\left(\frac{1}{2}+j\right)_{m}\left(\frac{1}{2}+j\right)_{n}\left(\frac{1}{2}+j\right)_{p}(2 \nu+1+j)_{2 p}},
$$

where we used the Pochhammer symbol $(x)_{n}=x(x+1) \cdots(x+n-1)=\frac{\Gamma(x+n)}{\Gamma(x)}$.

The quantities $\tau$ and $v$ are Schrödinger invariant quantities defined by

$$
\tau=\frac{t_{21} t_{43}}{t_{31} t_{42}}, \quad v_{123}=\frac{\left(t_{21} x_{32}-t_{32} x_{21}\right)^{2}}{2 t_{21} t_{32} t_{31}}, \quad v_{234}=\frac{\left(t_{32} x_{43}-t_{43} x_{32}\right)^{2}}{2 t_{32} t_{43} t_{42}} .
$$

They may be considered as the analogue of the cross-ratios, quantities invariant under the conformal symmetry, in usual CFT.

We note that an ansatz for Schrödinger invariant four-point functions is given in [73] which contains an arbitrary function of four Schrödinger invariant "cross-ratios" $(\tau$ and three $v$ 's). For one space and one time dimension, the number of independent Schrödinger invariant cross-ratios is decreased by one, ${ }^{14}$ so that there are three independent cross-ratios ( $\tau$ and two $v$ 's appearing in $(3.23)$ ).

The function (3.24) is symmetric in $v_{123}$ and $v_{234}$. The invariant quantity $v$, say, $v_{123}$ is proportional to the squared "area" of the triangle spanned by the space-time points 1 , 2,3 . Thus $v$ may be considered as measuring the degree of "non-collinearity" of the three space-time points. For instance, if the points 1-2-3 are collinear, one has $v_{123}=0$. In this case, (3.24) reduces to a double hypergeometric function,

$$
\mathcal{F}\left(0, v_{234}, \tau\right)=F_{0: 1: 3}^{1: 0: 3}\left[\begin{array}{c}
\frac{\nu}{2}+\frac{3}{4}:-; \frac{\nu}{2}+\frac{3}{4}, \frac{\nu}{2}+\frac{3}{4}, \frac{\nu}{2}+\frac{1}{4} \\
-\frac{1}{2} ; \frac{1}{2}, \quad \nu+1, \quad \nu+\frac{1}{2}
\end{array} \mid v_{234}, \tau\right],
$$

\footnotetext{
${ }^{14}$ Concretely, $\sqrt{v_{134}}$ can be written as a linear combination of $\sqrt{v_{123}}$ and $\sqrt{v_{234}}$ for one space and one time dimension.
} 
where the Kampé de Fériet series [74, p.27] is used. If all the four points lie along a line, it further reduces to a hypergeometric function of the single cross-ratio $\tau$,

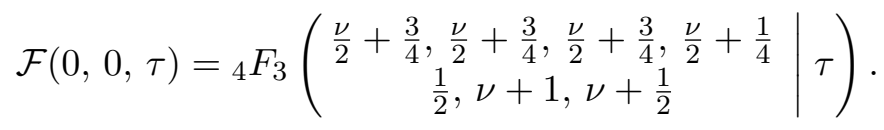

\section{OPE decomposition of the four-point function}

By taking various limits of the four-point function we have computed in the previous section, one can extract the information of OPE coefficients and a three-point function.

In section 4.1 we consider two different decompositions of the pairwise equal-time four-point function (3.13) using OPE. One of the decompositions, the "s-channel" decom-

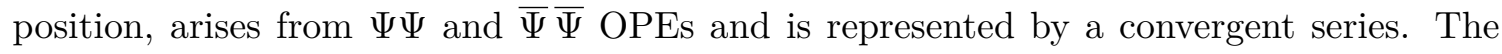
operators appearing in the intermediate channel have anomalous dimensions, i.e. their scaling dimensions depend on the coupling constant. The other decomposition, the "t-channel" decomposition, arises from two $\Psi \bar{\Psi}$ OPEs and is represented by an asymptotic series. By representing the same four-point function in two ways by OPE, we prove the operator associativity of the model, for this particular four-point function. The asymptotic series for the "t-channel" decomposition has also exponentially small correction terms, which also have an interpretation via OPE.

In section 4.2 we study in detail the "s-channel" decomposition and show that only one primary operator $\Phi$ appears in the $\Psi \Psi$ OPE. We fix the forms of the descendant operators of $\Phi$ appearing in the OPE and compute all the relevant OPE coefficients. In section 4.3 we compute the three-point function $\Psi \Psi \bar{\Phi}$ from the four-point function. In section 4.4 we discuss a peculiar property of the two-point functions between operators arising in the $\Psi \bar{\Psi}$-OPE.

\subsection{Decomposition of pairwise equal-time four-point function}

\subsection{1 "s-channel" decomposition}

We consider the expansion of the pairwise equal-time four-point function (3.13) in the parameter $x^{2} / t$, where $x$ refers to both $x_{21}$ and $x_{43}$. As it turns out, this expansion has an infinite convergence radius, so that the expansion is valid for an arbitrarily large value of $\frac{x^{2}}{t}$. The expansion becomes more useful when $\frac{x^{2}}{t} \ll 1$ since the first few terms will then dominate the series. The smaller the value of $\frac{x^{2}}{t}$, the closer are the operators $\Psi\left(t, x_{4}\right), \Psi\left(t, x_{3}\right)$ and $\bar{\Psi}\left(0, x_{2}\right), \bar{\Psi}\left(0, x_{1}\right)$ respectively. Therefore, considering this expansion should amount to considering the OPE between $\Psi\left(t, x_{4}\right) \Psi\left(t, x_{3}\right)$ and $\bar{\Psi}\left(0, x_{2}\right) \bar{\Psi}\left(0, x_{1}\right)$. (See (1.5).) Since the argument of the modified Bessel function is $z=\frac{x_{21} x_{43}}{2 t} \sim \frac{x^{2}}{t}$, one can use the definition of the modified Bessel function by the series expansion,

$$
I_{\nu}(z)=\left(\frac{1}{2} z\right)^{\nu} \sum_{k=0}^{\infty} \frac{\left(\frac{1}{4} z^{2}\right)^{k}}{k ! \Gamma(\nu+k+1)} .
$$


This series is convergent for any value of the argument $z$. Substituting (4.1) into (3.13), we obtain,

$$
\begin{aligned}
& \left\langle\Psi\left(t, x_{4}\right) \Psi\left(t, x_{3}\right) \bar{\Psi}\left(0, x_{2}\right) \bar{\Psi}\left(0, x_{1}\right)\right\rangle \\
& \quad=e^{-\frac{x^{2}}{t}} \sqrt{\frac{x_{21} x_{43}}{4 \pi t^{3}}}\left(\frac{x_{21} x_{43}}{4 t}\right)^{\nu} e^{-\frac{x_{21}^{2}+x_{43}^{2}}{4 t}} \sum_{k=0}^{\infty} \frac{\left(\frac{x_{21} x_{43}}{4 t}\right)^{2 k}}{k ! \Gamma(\nu+k+1)},
\end{aligned}
$$

where $X \equiv \frac{x_{3}+x_{4}-x_{1}-x_{2}}{2}$.

This decomposition of the four-point function emerges from the equal-time OPEs

$$
\begin{gathered}
\bar{\Psi}\left(0, x_{2}\right) \bar{\Psi}\left(0, x_{1}\right)=\sum_{k=0}^{\infty} C_{k} x_{21}^{\Delta_{k}-1} \bar{\Phi}_{k}\left(0, \frac{x_{1}+x_{2}}{2}\right), \\
\Psi\left(t, x_{4}\right) \Psi\left(t, x_{3}\right)=\sum_{k=0}^{\infty} C_{k} x_{43}^{\Delta_{k}-1} \Phi_{k}\left(t, \frac{x_{3}+x_{4}}{2}\right) .
\end{gathered}
$$

Here $C_{k}$ 's are the OPE coefficients, ${ }^{15}$ and $\Delta_{k}$ 's are dimensions of the operator $\Phi_{k}$ (and $\bar{\Phi}_{k}$ ). The operators $\bar{\Phi}_{k}$ are charge-2 operators, $N_{\bar{\Phi}_{k}}=2$. The powers of $x_{21}$ and $x_{43}$ in (4.3) and (4.4) are fixed by scale invariance. For simplicity, we will set $\left(x_{1}+x_{2}\right) / 2=0$, $\left(x_{3}+x_{4}\right) / 2=X$, using translational invariance. Equation (4.2) is valid provided $x_{21}>$ $0, x_{43}>0$ for both the bosonic and fermionic models. We assume in this subsubsection, without loss of generality, that these conditions are met.

In (4.3) and (4.4), we are not distinguishing primary and descendant operators. We will see that $\Phi_{0} \equiv \Phi$ is the only primary operator appearing in the OPE and all other operators $\Phi_{k}(k=1,2, \cdots)$ are its descendants in section 4.2 , where we also compute all OPE coefficients $C_{k}$ 's.

It is easy to read off the dimensions $\Delta_{k}$ by comparing the powers of $x_{21}$ and $x_{43}$ in the formulae (4.3) and (4.4) with (4.2). We obtain,

$$
\Delta_{k}=\frac{3}{2}+\nu+2 k
$$

We see that the scaling dimensions depend on the coupling constant $\nu$; the operators $\Phi_{k}$ 's have anomalous dimensions.

Let us consider the leading order contribution from the lowest-dimension operator $\Phi$ with dimension $\Delta_{0}=\frac{3}{2}+\nu$. From (4.3) and (4.4) we obtain

$$
\left\langle\Psi\left(t, x_{4}\right) \Psi\left(t, x_{3}\right) \bar{\Psi}\left(0, x_{2}\right) \bar{\Psi}\left(0, x_{1}\right)\right\rangle \approx C_{0}^{2} x_{43}^{\nu+\frac{1}{2}} x_{21}^{\nu+\frac{1}{2}}\langle\Phi(t, X) \bar{\Phi}(0,0)\rangle .
$$

Comparing this to the leading order term (both in the expansion by $x_{43}$ and $x_{21}$ ) of (4.2),

$$
\left\langle\Psi\left(t, x_{4}\right) \Psi\left(t, x_{3}\right) \bar{\Psi}\left(0, x_{2}\right) \bar{\Psi}\left(0, x_{1}\right)\right\rangle \approx \frac{1}{t^{\frac{3}{2}+\nu}} e^{-\frac{x^{2}}{t}} \times \frac{1}{4^{\nu} \sqrt{4 \pi} \Gamma(\nu+1)} x_{21}^{\nu+\frac{1}{2}} x_{43}^{\nu+\frac{1}{2}},
$$

we can read off the leading OPE coefficient to be

$$
C_{0}=\frac{1}{2^{\nu}(4 \pi)^{\frac{1}{4}} \sqrt{\Gamma(\nu+1)}}
$$

\footnotetext{
${ }^{15}$ We take $C_{k}$ to be real by choosing the phases of $\Phi_{k}$ 's appropriately.
} 
together with the two-point function

$$
\langle\Phi(t, X) \bar{\Phi}(0,0)\rangle=\frac{1}{t^{\frac{3}{2}+\nu}} e^{-\frac{X^{2}}{t}} .
$$

The spacetime dependence agrees with the general form of the two-point function of the primary operator (1.4) with $N_{\bar{\Phi}}=2$ and $\Delta=\Delta_{0}=\frac{3}{2}+\nu$. We fixed the normalisation of $\Phi$ by (4.9). We will see presently that $\Phi$ is indeed a primary operator. The differences in scaling dimensions of $\Phi_{k}(k>0)$ and $\Phi$ are integers. This suggests that $\Phi_{k}(k>0)$ are descendants of $\Phi$. We will see later in section 4.2 that this is the case.

In section 3.1, we computed the four-point function by identifying it with the twoparticle propagator. In this identification, the expansion parameters $x_{43}$ and $x_{21}$ are the relative coordinates, and $X$ appearing above is the difference in the final and the initial centre of mass position. Thus, the "s-channel" OPE decomposition described here may be interpreted as representing the separation of the centre of mass and relative motions.

The state-operator map. The spectrum of operators (4.5) is consistent with the stateoperator map introduced by Nishida and Son for Schrödinger invariant theories [44]. (See also [45].) The state-operator map is a one-to-one correspondence between an operator $\overline{\mathcal{O}}$ (with positive $\mathrm{U}(1)$ charge $N_{\overline{\mathcal{O}}}>0$ ) of a Schrödinger invariant theory and a state $|\overline{\mathcal{O}}\rangle$ of the model which is obtained by adding an external harmonic oscillator potential to the theory. ${ }^{16}$ For our model, the extra harmonic oscillator term can be represented as an additional contribution

$$
S_{\text {ext }}=-\int d t d x \frac{1}{2} x^{2}|\Psi|^{2}
$$

to the action (2.4). The state-operator map has the property that the scaling dimension of $\overline{\mathcal{O}}$ equals the energy of the state $|\overline{\mathcal{O}}\rangle$ in the deformed model. The map also preserves the

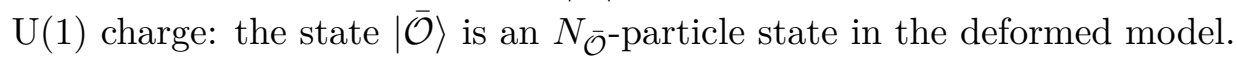

The energy spectrum of the deformed model can be exactly computed. This is the celebrated result by Calogero [42]. For $N$-particle state, it is,

$$
E=\frac{N}{2}+\left(\nu+\frac{1}{2}\right) \frac{N(N-1)}{2}+\sum_{i=1}^{N} n_{i},
$$

where $n_{i}(i=1, \cdots, N)$ are integers satisfying

$$
0 \leq n_{1} \leq n_{2} \leq \cdots \leq n_{N}
$$

for both the bosonic and fermionic models. (See, for example, (16) of [60].) Thus, via the state-operator map, we have the complete tabulation of operators (with positive U(1)

\footnotetext{
${ }^{16}$ The state-operator map first appeared in [75] for the free-field theory. For a specific interacting model (the fermion at unitarity), Werner and Castin applied a similar map between the theory with and without the external harmonic oscillator potential [76]. We note also that the state-operator maps given in [44] and [45] are slightly different. The map given in [45] has the advantage that the operator $\left[K_{i}, \overline{\mathcal{O}}\right]$ is directly mapped to the state $K_{i}|\mathcal{O}\rangle$.
} 
charge ${ }^{17}$ ) including their correct multiplicities. The scaling dimensions are given simply by (4.11), with the identification $\Delta=E$.

Let us consider the simplest case, $N=1$. The operators are labelled by a single non-negative integer $n$ and their dimensions are

$$
\Delta=\frac{1}{2}+n \text {. }
$$

The lowest dimension operator necessarily is a primary operator, which is nothing but the fundamental field $\bar{\Psi}$. (Recall that the scaling dimension of the fundamental field is protected and equals $\frac{1}{2}$. See the explanation below (2.9).) The operators with $n>0$ are descendants of the fundamental field, $\partial_{x}^{n} \bar{\Psi}$. It is not necessary to consider descendants produced by acting with $\partial_{t}$ 's on $\bar{\Psi}$. This is because it is redundant to consider the null operator $\left(\partial_{t}-\frac{1}{2} \partial_{x}^{2}\right) \Psi$ in the OPE. See [30, 31, 52].

For the $N=2$ case, which is relevant for the "s-channel" OPE we are considering, (4.11) becomes

$$
\Delta=\frac{3}{2}+\nu+n_{1}+n_{2},
$$

with $0 \leq n_{1} \leq n_{2} \cdot{ }^{18}$ This includes the spectrum found from the OPE, (4.5), consistently with the correct coupling constant dependence of the scaling dimensions. We now see that the operator $\bar{\Phi}$ with dimension $\Delta_{0}=\frac{3}{2}+\nu$ has the lowest scaling dimension in the charge- 2 sector, and must therefore be a primary operator. (In (4.5) the scaling dimensions are separated by even integers whereas in (4.14) the separations are general integers. This difference arises because we are defining the OPE at the symmetric points $\frac{x_{1}+x_{2}}{2}, \frac{x_{3}+x_{4}}{2}$ in (4.3) and (4.4).)

One can also characterise primary operators using the state-operator map: they correspond to the states annihilated by the charges $C, K{ }^{19}$ It should be possible to directly study this condition in the Calogero model (with the harmonic oscillator external potential). This will lead to a complete classification of the primary operators (with nonzero charge, since the zero-charge sector defies the use of the state-operator map). The operator technique developed in [77] seems to be well-suited for this purpose. This problem will be addressed in a separate publication.

\subsection{2 "t-channel" decomposition}

Next, we consider the pairwise equal-time four-point function $\left\langle\Psi\left(t, x_{4}\right) \Psi\left(t, x_{3}\right) \bar{\Psi}\left(0, x_{2}\right) \bar{\Psi}\left(0, x_{1}\right)\right\rangle$ in the regime in which $x^{2} / t$ is large, where $x$ refers collectively to $x_{21}>0$ and $x_{43}>0$. In this regime, the spacetime points 2,4 and 1,3 can be made close to each other, respectively. Hence we expect that this regime should be understood from the OPEs $\Psi\left(t, x_{4}\right) \bar{\Psi}\left(0, x_{2}\right)$ and $\Psi\left(t, x_{3}\right) \bar{\Psi}\left(0, x_{1}\right)$. (See (1.6).)

\footnotetext{
${ }^{17}$ Of course, negatively charged operators are also classified since they are the complex conjugates of positively charged operators. The state-operator map fails to capture, importantly, charge-zero operators (operators with $N_{\mathcal{O}}=0$ ).

${ }^{18}$ For the free-field theory case, $\nu=-\frac{1}{2}$, one can understand this spectrum as that of the operators $\partial_{x}^{n_{1}} \bar{\Psi} \partial_{x}^{n_{2}} \bar{\Psi}$.

${ }^{19}$ For our notation about the Schrödinger algebra, see appendix A.
} 
One can use, for the four-point function (3.13), the asymptotic expansion of the modified Bessel function [78, (10.40.1), (10.17.1)]

$$
I_{\nu}(z) \sim \frac{e^{z}}{(2 \pi z)^{\frac{1}{2}}} \sum_{p=0}^{\infty}(-1)^{p} \frac{a_{p}(\nu)}{z^{p}}
$$

where $a_{0}(\nu)=1$ and

$$
a_{p}(\nu)=\frac{\left(4 \nu^{2}-1^{2}\right)\left(4 \nu^{2}-3^{2}\right) \cdots\left(4 \nu^{2}-(2 p-1)^{2}\right)}{p ! 8^{p}} .
$$

The asymptotic expansion is valid in the limit $z \rightarrow \infty$ for $|\mathrm{ph} z| \leq \frac{\pi}{2}-\epsilon$, which includes $z>0$ relevant for us. (ph $z$ is the argument of $z$ and $\epsilon$ is a positive infinitesimal quantity.) Substituting (4.15) to (3.13), we obtain

$$
\begin{aligned}
& \left\langle\Psi\left(t, x_{4}\right) \Psi\left(t, x_{3}\right) \bar{\Psi}\left(0, x_{2}\right) \bar{\Psi}\left(0, x_{1}\right)\right\rangle \\
& \quad=e^{-\frac{x_{21}^{2}+x_{43}^{2}+\left(x_{1}+x_{2}-x_{3}-x_{4}\right)^{2}}{4 t}} \times \sqrt{\frac{x_{21} x_{43}}{4 \pi t^{3}}} e^{\frac{x_{21} x_{43}}{2 t}} \frac{1}{\sqrt{2 \pi \frac{x_{21} x_{43}}{2 t}}} \sum_{p=0}^{\infty}(-1)^{p} \frac{a_{p}(\nu)}{\left(\frac{x_{21} x_{43}}{2 t}\right)^{p}} .
\end{aligned}
$$

It is essential that the exponential factors in this formula combine to yield

$$
e^{-\frac{x_{21}^{2}+x_{43}^{2}+\left(x_{3}+x_{4}-x_{1}-x_{2}\right)^{2}}{4 t}} e^{\frac{x_{21} x_{43}}{2 t}}=e^{-\frac{x_{31}^{2}+x_{42}^{2}}{2 t}} .
$$

We obtain

$$
\begin{aligned}
& \left\langle\Psi\left(t, x_{4}\right) \Psi\left(t, x_{3}\right) \bar{\Psi}\left(0, x_{2}\right) \bar{\Psi}\left(0, x_{1}\right)\right\rangle \\
& \quad=\frac{1}{2 \pi} e^{-\frac{x_{31}^{2}+x_{42}^{2}}{2 t}} \times \frac{1}{t} \sum_{p=0}^{\infty}(-1)^{p} 2^{p} a_{p}(\nu) \frac{t^{p}}{x_{21}^{p} x_{43}^{p}} .
\end{aligned}
$$

To clarify the connection to the OPE, we define

$$
X^{\prime}=\frac{x_{4}+x_{2}}{2}-\frac{x_{3}+x_{1}}{2} .
$$

Then we have, using $x_{21}=X^{\prime}-\frac{x_{42}-x_{31}}{2}$ and $x_{43}=X^{\prime}+\frac{x_{42}-x_{31}}{2}$,

$$
\begin{aligned}
& \left\langle\Psi\left(t, x_{4}\right) \Psi\left(t, x_{3}\right) \bar{\Psi}\left(0, x_{2}\right) \bar{\Psi}\left(0, x_{1}\right)\right\rangle \\
& \quad=\frac{1}{2 \pi} e^{-\frac{x_{31}^{2}+x_{42}^{2}}{2 t}} \times \frac{1}{t} \sum_{p=0}^{\infty}(-1)^{p} 2^{p} a_{p}(\nu) \frac{t^{p}}{\left(X^{\prime 2}-\frac{\left(x_{42}-x_{31}\right)^{2}}{4}\right)^{p}} .
\end{aligned}
$$

This "t-channel" decomposition of the four-point function is valid for $x_{21}>0, x_{43}>0$ for both the bosonic and fermionic models. Hereafter in this subsubsection, to be specific, we consider the bosonic model.

The decomposition (4.21) indeed has the form which arises from the OPEs $\Psi\left(t, x_{4}\right) \bar{\Psi}\left(0, x_{2}\right)$ and $\Psi\left(t, x_{3}\right) \bar{\Psi}\left(0, x_{1}\right)$. In particular, it shows that the OPE $\Psi(t, x) \bar{\Psi}\left(0, x^{\prime}\right)$ is well-defined in the limit $t \rightarrow 0$ with fixed $\frac{\left(x-x^{\prime}\right)^{2}}{t}$. More precisely, the OPE has the form

$$
\begin{aligned}
\Psi(t, x) \bar{\Psi}\left(0, x^{\prime}\right) & =\sum_{k=0}^{\infty} \tilde{C}_{k}\left(t, x-x^{\prime}\right) \mathcal{J}_{k}\left(\frac{t}{2}, \frac{x+x^{\prime}}{2}\right) \\
& =\sum_{k=0}^{\infty}\left(x-x^{\prime}\right)^{\tilde{\Delta}_{k}-1} f_{k}\left(\frac{\left(x-x^{\prime}\right)^{2}}{t}\right) \mathcal{J}_{k}\left(\frac{t}{2}, \frac{x+x^{\prime}}{2}\right),
\end{aligned}
$$


where $\mathcal{J}_{k}$ (with scaling dimension $\tilde{\Delta}_{k}$ ) are the operators in the intermediate channel. In the second line, we have used the scale invariance to constrain the OPE coefficients $\tilde{C}_{k}$. The two-point functions of $\mathcal{J}_{k}$ 's are

$$
\left\langle\mathcal{J}_{k}\left(0, X^{\prime}\right) \mathcal{J}_{k^{\prime}}(0,0)\right\rangle=\frac{D_{k k^{\prime}}}{X^{\prime \tilde{\Delta}_{k}+\tilde{\Delta}_{k^{\prime}}}} .
$$

Some of the coefficients $D_{k k^{\prime}}$ can be absorbed into the normalisation of the operators $\mathcal{J}_{k}$. Here, we are not specifying whether $\mathcal{J}_{k}$ is a primary or a descendant operator. (They can be a linear combination of primary and descendant operators in general.) The operators $\mathcal{J}_{k}$ have vanishing $\mathrm{U}(1)$ charges. Note that the primary and descendant operators for the charge-zero sector behave differently from those in other sectors [52]. This is because the generators $K$ and $P$, which act as the "ladder operators" for the sectors with the nonzero $\mathrm{U}(1)$ charge, commute for the charge-zero sectors. (The commutation relations of the Schrödinger algebra are given in appendix A.)

From the OPE (4.22) and the two-point functions (4.23), we obtain

$$
\begin{aligned}
& \left\langle\Psi\left(t, x_{4}\right) \Psi\left(t, x_{3}\right) \bar{\Psi}\left(0, x_{2}\right) \bar{\Psi}\left(0, x_{1}\right)\right\rangle \\
& \quad=\sum_{m=0}^{+\infty} \sum_{n=0}^{+\infty} x_{42}^{\tilde{\Delta}_{m}-1} x_{31}^{\tilde{\Delta}_{n}-1} f_{m}\left(\frac{x_{42}^{2}}{t}\right) f_{n}\left(\frac{x_{31}^{2}}{t}\right) D_{m n} \frac{1}{X^{\prime \tilde{\Delta}_{m}+\tilde{\Delta}_{n}}} .
\end{aligned}
$$

The powers of $X^{\prime}, x_{21}$, and $x_{43}$ are all integers in (4.19). Comparing (4.19) and (4.24), we see that this strongly suggests that $\tilde{\Delta}_{k}$ 's are also integers. There are ambiguities, however, which stem from the fact that one can insert

$$
1=\left(\frac{x_{42}^{2}}{t}\right)^{\delta}\left(\frac{t}{x_{31}^{2}}\right)^{\delta}\left(\frac{x_{31}^{2}}{x_{42}^{2}}\right)^{\delta},
$$

where $\delta$ is an arbitrary number, into (4.24). This leads to a redefinition of $f_{m}$ and $f_{n}$ and a shift of the dimensions $\tilde{\Delta}_{m}$ and $\tilde{\Delta}_{n}$ by $\pm 2 \delta$. We will return to a possible resolution of this ambiguity towards the end of this subsubsection. Although it seems unlikely that a set of consistent OPE coefficients exist with non-integer valued $\tilde{\Delta}_{m}>0$, we have not succeeded in ruling this possibility out. We hereafter assume that the scaling dimensions are integers and write

$$
\tilde{\Delta}_{k}=k
$$

We can fix the first two OPE coefficients, $f_{0}$ and $f_{1}$, by comparing (4.19) and (4.24) under this assumption. Firstly, we see that the lowest dimension operator $\mathcal{J}_{0}$ has scaling dimension $0,{ }^{20}$ and hence should be identified with the identity operator $\mathcal{J}_{0}=1$. This implies $D_{00}=1$ and $D_{0 n}=0(n>0)$ since the one-point function of any operator with nonzero dimension vanishes because of scale invariance. The OPE coefficient is

$$
f_{0}\left(\frac{x^{2}}{t}\right)=e^{-\frac{x^{2}}{2 t}} \frac{x}{\sqrt{2 \pi t}},
$$

\footnotetext{
${ }^{20}$ This conclusion is not affected by the ambiguity associated with (4.25).
} 
and hence the leading order term in the $\Psi \bar{\Psi} \mathrm{OPE}$ is

$$
\Psi(0, t) \bar{\Psi}(0,0)=e^{-\frac{x^{2}}{2 t}} \frac{1}{\sqrt{2 \pi t}} \times 1+\ldots
$$

This is an expected result in view of the two-point function (2.9).

The subleading OPE coefficient can also be read off. We obtain

$$
f_{1}^{2} D_{11}=-\frac{1}{\pi} a_{1}(\nu) e^{-\frac{x^{2}}{t}},
$$

where $a_{1}(\nu)$ is given by (4.16). The operator $\mathcal{J}_{1}$ has dimension 1 and it is natural to identify it with the density of the $\mathrm{U}(1)$ charge. We have not fixed the normalisation of $\mathcal{J}_{1}$; this is the reason why both $f_{1}$ and $D_{11}$ appear in the above formula. By redefining the operator $\mathcal{J}_{1}$ appropriately by multiplying it by a phase factor, we can choose $f_{1}$ to be real and $\mathcal{J}_{1}$ to be hermitian.

Note that $a_{1}(\nu)$ flips its sign at $\nu=\frac{1}{2}$. The coefficient of the two-point function $\left\langle\mathcal{J}_{1}(0, x) \mathcal{J}_{1}(0,0)\right\rangle, D_{11}$, is positive for $\nu<\frac{1}{2}$ and negative for $\nu>\frac{1}{2}$. This does not contradict the unitarity of the theory. (The unitarity of the theory is guaranteed as the Hamiltonian of the model is hermitian.) We recall that, for the isotropic case, the positivity of the two-point function in a unitary CFT is proven via the state-operator map. The analogous state-operator map in $z=2$ Schrödinger invariant theory is not applicable to the charge-zero sector. Furthermore, one cannot invoke the positivity of the norm of the Hilbert space via $\left\langle\mathcal{J}_{m}(t, x) \mathcal{J}_{n}(0,0)\right\rangle=\left\langle 0\left|\mathcal{J}_{m}(t, x) \mathcal{J}_{n}(0,0)\right| 0\right\rangle$, since one can show that this equation holds for $t>0$ but not for $t=0$, which is the case of interest here. This point will be explained in section 4.4.

The special point $\nu=\frac{1}{2}$, where $f_{1}^{2} D_{11}=0$, corresponds to the point at which the Calogero model coincides with the system of free fermions; the asymptotic series (4.15) truncates at that point. Similar truncations of the asymptotic series occur also at $\nu=-\frac{1}{2}$ and at $\nu=\frac{3}{2}, \frac{5}{2}, \cdots$. The $\Psi \bar{\Psi}$-OPE appears to be degenerate for these special points.

It is not possible to fix the higher OPE coefficients $f_{n}(n=2,3, \cdots)$ unambiguously from the pairwise equal-time four-point function. This is because of the ambiguity associated with (4.25) (where $\delta$ is chosen to be an integer). Starting from the pairwise equal-time point-function, we are forced to take the coincident limit of both pairs of the spacetime points $(1,3)$ and $(2,4)$. (Note that we have to take the limit $\left(t_{31}, x_{31}\right) \rightarrow 0,\left(t_{42}, x_{42}\right) \rightarrow 0$ with fixed $\frac{x_{31}^{2}}{t_{31}}, \frac{x_{42}^{2}}{t_{42}}$ in order to have a well-defined $\Psi \bar{\Psi}$ OPE.) We should obtain more information on OPE coefficients from the general four-point function discussed in sections 3.2 and 3.3 , since then we can, say, pinch the spacetime points $(1,3)$ while keeping $(2,4)$ unpinched. By studying this type of limit, we expect to get the complete OPE coefficients and understanding of the primary/descendant structure of operators $\mathcal{J}_{k}$. This will be left as a future problem.

It is possible to read off some properties of the operators $\mathcal{J}_{k}$ without going into the details of the expression of the general four-point function. In particular, we find that the operators $\mathcal{J}_{k}(k \geq 1)$ have a rather unusual property, namely, that their two-point functions vanish unless the two operators are inserted on the same time slice. This will be shown in section 4.4. 
To summarise this subsubsection, we have shown that the pairwise equal-time fourpoint function (3.13) can be decomposed by using two $\Psi \bar{\Psi}$ OPEs. The decomposition (4.21) is represented by an asymptotic series rather than a convergent series. The charge-zero operators $\mathcal{J}_{m}$ appearing in the intermediate channels appear to have integer-valued scalar dimensions. $\mathcal{J}_{0}$ is the identity operator. We have computed the leading and the next-toleading OPE coefficients associated with $\mathcal{J}_{0}$ and $\mathcal{J}_{1}$.

Since we have represented the same four-point function now in two ways as "the schannel" decomposition (in section 4.1.1) and "the t-channel" decomposition here, we have thereby shown the operator associativity for this model, for the particular four-point function. Schematically, we have shown

$$
\left.\sum \gamma_{\bar{\Psi}_{1}}^{\Psi_{3}}=\left\langle\Psi\left(t, x_{4}\right) \Psi\left(t, x_{3}\right) \bar{\Psi}\left(0, x_{2}\right) \bar{\Psi}\left(0, x_{1}\right)\right\rangle=\sum_{\bar{\Psi}_{1}}^{\Psi_{4}}\right\rangle_{\bar{\Psi}_{2}}^{\Psi_{3}} .
$$

The second equality has to be understood as the representation of a function by an asymptotic series.

\subsubsection{The exponentially small corrections and "u-channel" contributions}

The asymptotic expansion of the modified Bessel function (4.15), and hence the "t-channel" decomposition of the four-point function, comes with exponentially small contributions. Here we will show that these correction terms can also be interpreted using the OPE.

As we shall see below, it is necessary to analytically continue the time variable $t$. We write

$$
t=e^{i \alpha} t^{\prime}
$$

where $\alpha \in \mathbb{R}$ and $t^{\prime}>0$. We will only consider the regime $0 \leq \alpha \leq \frac{\pi}{2}$. We consider the analytically continued pairwise equal-time four-point function defined by

$$
\left\langle 0\left|\Psi\left(t, x_{4}\right) \Psi\left(t, x_{3}\right) \bar{\Psi}\left(0, x_{2}\right) \bar{\Psi}\left(0, x_{1}\right)\right| 0\right\rangle
$$

where the operator in the Heisenberg picture, $\Psi(t, x)$, is given by

$$
\Psi(t, x)=e^{H t} \Psi(0, x) e^{-H t} .
$$

For $\alpha=0$, this definition coincides with the four-point function with the Euclidean time we have been considering in this paper. The computation in section 3.1 (and appendix B) goes through for the analytically continued case, and hence the result (3.13) is unaffected in form,

$$
\begin{aligned}
& \left\langle 0\left|\Psi\left(t, x_{4}\right) \Psi\left(t, x_{3}\right) \bar{\Psi}\left(0, x_{2}\right) \bar{\Psi}\left(0, x_{1}\right)\right| 0\right\rangle \\
& \quad=e^{-\frac{x_{21}^{2}+x_{43}^{2}+\left(x_{3}+x_{4}-x_{1}-x_{2}\right)^{2}}{4 t}} \times \sqrt{\frac{x_{21} x_{43}}{4 \pi t^{3}}} I_{\nu}\left(\frac{x_{21} x_{43}}{2 t}\right),
\end{aligned}
$$

where we assume $x_{21}>0, x_{43}>0$. 
By gradually increasing $\alpha$ from 0 to $\frac{\pi}{2}$, we have $t=i t^{\prime}$ and we obtain the four-point function of the theory with the "Minkowski" time: the analytically continued four-point function, considered as a function of $t^{\prime}$, coincides with the four-point function of the theory with the "Minkowski" time $t^{\prime}$. Let us write explicitly the four-point function for this case,

$$
\begin{aligned}
\left\langle\Psi\left(t^{\prime}, x_{4}\right) \Psi\left(t^{\prime}, x_{3}\right) \bar{\Psi}\left(0, x_{2}\right) \bar{\Psi}\left(0, x_{1}\right)\right\rangle_{\mathrm{M}} \\
\quad=\left\langle 0\left|\Psi_{\mathrm{M}}\left(t^{\prime}, x_{4}\right) \Psi_{\mathrm{M}}\left(t^{\prime}, x_{3}\right) \bar{\Psi}\left(0, x_{2}\right) \bar{\Psi}\left(0, x_{1}\right)\right| 0\right\rangle \\
\quad=e^{i \frac{x_{21}^{2}+x_{43}^{2}+\left(x_{3}+x_{4}-x_{1}-x_{2}\right)^{2}}{4 t^{\prime}}} \times e^{-i \frac{3}{4} \pi} \sqrt{\frac{x_{21} x_{43}}{4 \pi t^{\prime 3}}} I_{\nu}\left(-i \frac{x_{21} x_{43}}{2 t^{\prime}}\right) \\
\quad=e^{i \frac{x_{21}^{2}+x_{43}^{2}+\left(x_{3}+x_{4}-x_{1}-x_{2}\right)^{2}}{4 t^{\prime}}} \times e^{-i \frac{\pi}{2}\left(\nu+\frac{3}{2}\right)} \sqrt{\frac{x_{21} x_{43}}{4 \pi t^{\prime 3}}} J_{\nu}\left(\frac{x_{21} x_{43}}{2 t^{\prime}}\right) .
\end{aligned}
$$

Here, the subscript M refers to the theory in the "Minkowski" signature and the Heisenberg operator assumes the usual quantum mechanical form, $\Psi_{\mathrm{M}}\left(t^{\prime}, x_{4}\right)=e^{i H t^{\prime}} \Psi(0, x) e^{-i H t^{\prime}}$. We used $I_{\nu}(z)=e^{-\frac{i \pi \nu}{2}} J_{\nu}\left(e^{i \frac{\pi}{2}} z\right)[78,(10.27 .6)]$. The expression (4.35), of course, can also be obtained directly for the Minkowski theory without relying on the analytic continuation.

In the regime $0<\alpha \leq \frac{\pi}{2}$, the asymptotic expansion accompanied with exponentially small correction terms $[78,(10.40 .5)]$,

$$
I_{\nu}(z) \sim \frac{e^{z}}{(2 \pi z)^{\frac{1}{2}}} \sum_{p=0}^{\infty}(-1)^{p} \frac{a_{p}(\nu)}{z^{p}}-i e^{-\nu \pi i} \frac{e^{-z}}{(2 \pi z)^{\frac{1}{2}}} \sum_{p=0}^{\infty} \frac{a_{p}(\nu)}{z^{p}},
$$

captures the modified Bessel function $I_{\nu}(z)$ accurately. The coefficients $a_{p}(\nu)$ are defined in (4.16). The first term in (4.36) coincides with the asymptotic expansion (4.15) used for the "t-channel" decomposition in section 4.1.2.

The expression (4.36) is not valid for the Euclidean theory $(\alpha=0)$. The reason is that $\alpha=0$ corresponds to a Stokes line of the modified Bessel function $I_{\nu}(z)$, where the first term in (4.36) is maximally dominating over the second term. ${ }^{21}$ As is well known, across the Stokes line, the coefficients of the smaller terms change almost discontinuously albeit in a controlled manner [79]. One needs to use a specially tailored expansion formula to study the behaviour of a function exactly on the Stokes line. Such a formula for $I_{\nu}(z)$ was derived in [80]. We found a natural interpretation in terms of OPE for the formula (4.36) rather than the expansion valid exactly on the Stokes line given in [80]. This may suggest that it is useful to define the Euclidean theory not exactly at $\alpha=0$ but rather using the limit $\alpha \rightarrow 0$. Note that, for large $|z|$, one needs only small $\alpha>0$ to make the expansion (4.36) accurate.

Substituting (4.36) into (3.13), we find that the exponentially small corrections to the four-point function $\left\langle\Psi\left(t, x_{4}\right) \Psi\left(t, x_{3}\right) \bar{\Psi}\left(0, x_{2}\right) \bar{\Psi}\left(0, x_{1}\right)\right\rangle$ are

$$
-i e^{-i \pi \nu} \times \frac{1}{2 \pi} e^{-\frac{x_{41}^{2}+x_{32}^{2}}{2 t}} \frac{1}{t}\left(\sum_{p=0}^{\infty} 2^{p} a_{p}(\nu) \frac{t^{p}}{x_{21}^{p} x_{43}^{p}}\right) .
$$

This formula is valid for $x_{21}>0, x_{43}>0$ for both the bosonic and fermionic models. Hereafter in this subsubsection, we focus on the bosonic model.

\footnotetext{
${ }^{21}$ The Minkowski case $\alpha=\frac{\pi}{2}$ corresponds to an anti-Stokes line where the second and first terms are of comparable size. For $\alpha>\frac{\pi}{2}$, the second term becomes exponentially large compared to the first term.
} 
The similarity of (4.37) with the "t-channel" decomposition (4.19) is clear. In particular, the exponential factor in (4.36) and (3.13) combines in a similar manner to (4.18) and yields the exponential factor $e^{-\frac{x_{41}^{2}+x_{32}^{2}}{2 t}}$ in (4.37). Comparing this exponential factor with the corresponding factor, $e^{-\frac{x_{31}^{2}+x_{42}^{2}}{2 t}}$, in (4.19), we find that the roles of spacetime points 1 and 2 (or equivalently 3 and 4 ) are interchanged. This leads us to identify the exponentially small contributions (4.37) as arising from the OPEs $\Psi\left(t, x_{3}\right) \bar{\Psi}\left(0, x_{2}\right)$ and $\Psi\left(t, x_{4}\right) \bar{\Psi}\left(0, x_{1}\right)$. Schematically these contributions can be represented as,

$$
\sum \sum_{\Psi_{1}}^{\Psi_{3}} \Psi_{\Psi_{2}}^{\Psi_{4}}
$$

This interpretation can be made more precise. To clarify the connection to the OPE, we define

$$
X^{\prime \prime}=\frac{x_{4}+x_{1}}{2}-\frac{x_{3}+x_{2}}{2} .
$$

Then (4.37) becomes, using $x_{21}=-X^{\prime \prime}+\frac{x_{41}-x_{32}}{2}$ and $x_{43}=X^{\prime \prime}+\frac{x_{41}-x_{32}}{2}$,

$$
e^{-i \pi\left(\nu+\frac{1}{2}\right)} \times \frac{1}{2 \pi} e^{-\frac{x_{41}^{2}+x_{32}^{2}}{2 t}} \times \frac{1}{t} \sum_{p=0}^{\infty}(-1)^{p} 2^{p} a_{p}(\nu) \frac{t^{p}}{\left(X^{\prime \prime 2}-\frac{\left(x_{41}-x_{32}\right)^{2}}{4}\right)^{p}} .
$$

Note that an extra factor $(-1)^{p}$ appeared in the summand, compared to (4.37), due to the rewriting in terms of the variable $X^{\prime \prime}$. Now we see that (4.40) have precisely the same form, except for the overall phase factor $e^{-i \pi\left(\nu+\frac{1}{2}\right)}$, to the "t-channel" decomposition (4.21). This is natural since both terms originate from the $\Psi \bar{\Psi}$-OPE.

The overall phase factor has a natural interpretation within the framework of the generalised statistics $[60,61]$ for the Calogero model. The generalised statistics is an interesting way of understanding various properties of the Calogero model as a consequence of the phase factor $e^{-i \pi\left(\nu+\frac{1}{2}\right)}$ associated with each exchange of two particles. We indeed see that the "u-channel" terms which are obtained by the exchange of, say, the two particles at the spacetime points 1 and 2, acquire precisely that phase factor relative to the "t-channel" terms.

The successful interpretation of the exponentially small terms as the "u-channel" contributions relies on the fact that the coefficients of the first and the second terms of (4.36) are closely related. (Both are given in terms of $a_{p}(\nu)$ defined by (4.16).) This connection is an example of the so-called resurgence phenomenon. (See, for example, [81].) Thus the resurgence property of the modified Bessel function represents the fact that both " $\mathrm{t}$ channel" and "u-channel" contributions arise from the $\Psi \bar{\Psi}$ OPE.

There is another way of understanding the necessity of the resurgence property and the role of the " $\mathrm{u}$-channel" terms from the point of view of the OPE. When $\nu$ is a half-odd integer (i.e. $\nu=-\frac{1}{2}, \frac{1}{2}, \frac{3}{2}, \frac{5}{2}, \cdots$ ), the asymptotic series (4.36) truncates and becomes exact. (The Stokes phenomenon does not occur for these values of $\nu$.) The four-point function 
(in Euclidean time) becomes, writing $\nu=n+\frac{1}{2}$ with $n=0,1, \cdots,{ }^{22}$

$$
\begin{aligned}
& \left\langle\Psi\left(t, x_{4}\right) \Psi\left(t, x_{3}\right) \bar{\Psi}\left(0, x_{2}\right) \bar{\Psi}\left(0, x_{1}\right)\right\rangle \\
& \quad=e^{-\frac{x_{21}^{2}+x_{43}^{2}+\left(x_{3}+x_{4}-x_{1}-x_{2}\right)^{2}}{4 t}} \times \frac{x_{21} x_{43}}{2 \pi t^{2}} \times \mathrm{i}_{n}^{(1)}\left(\frac{x_{21} x_{43}}{2 t}\right)
\end{aligned}
$$

where $\mathrm{i}_{n}^{(1)}(z)$ is a modified spherical Bessel function defined by [78, (10.49.8)]

$$
\mathrm{i}_{n}^{(1)}(z)=\frac{1}{2} e^{z} \sum_{k=0}^{n}(-1)^{k} \frac{a_{k}\left(n+\frac{1}{2}\right)}{z^{k+1}}+(-1)^{n+1} \frac{1}{2} e^{-z} \sum_{k=0}^{n} \frac{a_{k}\left(n+\frac{1}{2}\right)}{z^{k+1}},
$$

which is related to $I_{\nu}(z)$ by $[78,(10.47 .7)]$,

$$
\mathbf{i}_{n}^{(1)}(z)=\sqrt{\frac{1}{2} \pi / z} I_{n+\frac{1}{2}}(z) .
$$

The first and the second finite sum in (4.42) correspond to the exponentially large and small contributions in (4.36), respectively. Since these formulae are valid for all $z=$ $\frac{x_{43} x_{21}}{2 t}$, one can in particular consider the limit $z \rightarrow 0$. This limit corresponds to the limit where spacetime points $(1,2)$ or $(3,4)$ become coincident (related to the "s-channel" decomposition studied in section 4.1.1). Although each term in the first and second sum in (4.42) diverges, there are cancellations between these terms such that $\mathrm{i}_{n}^{(1)}(z) \sim z^{n}$ for $z \rightarrow 0$. This must be the case. Consider, say, the limit $x_{43} \rightarrow 0$, in which $z$ also goes to zero, $z \sim x$. In this limit, the four-point function is controlled by the OPE (4.4), $\Psi(0, x) \Psi(0,0) \sim x^{n+1} \Phi$. (Note that the scaling dimensions of the operators $\Psi$ and $\Phi$ are $\frac{1}{2}$ and $n+2$, respectively.) Hence the four-point function behaves as $x_{43}^{n+1}$. This agrees with (4.41) and (4.42) together with $\mathrm{i}_{n}^{(1)}(z) \sim z^{n}$. The consistency of the four-point function with the $\Psi \bar{\Psi}$ OPE relies on the cancellations, which in turn occur because of the resurgence relations, i.e. the relations between the coefficients of the exponentially small and large terms of (4.36).

It is intriguing that the interpretation of the exponentially small terms as the " $\mathrm{u}$ channel" contributions means that the operator associativity relation (4.30) can be made more accurate by including "u-channel" contributions. Schematically, we have,

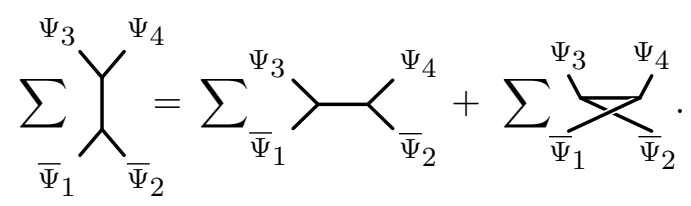

\subsection{Detailed analysis of "s-channel" decomposition}

In this subsection, we take a closer look into the "s-channel" OPE decomposition of the pairwise equal-time four-point function (3.13) which arises when we consider the OPE of $\bar{\Psi}\left(0, x_{2}\right) \bar{\Psi}\left(0, x_{1}\right)$ and of $\Psi\left(t, x_{4}\right) \Psi\left(t, x_{3}\right)$. In section 4.1.1, we have seen that the operators $\Phi_{k}$ appearing in the $\Psi \Psi$ OPE, (4.4), have dimensions,

$$
\Delta_{k}=\frac{3}{2}+\nu+2 k, \quad(k=0,1,2, \cdots),
$$

\footnotetext{
${ }^{22}$ Some formulae for the free-boson case, $\nu=-\frac{1}{2}$, are presented in appendix E.
} 
and the lowest dimension operator $\Phi=\Phi_{0}$ is a primary operator. We also obtained the leading OPE coefficient (4.8) involving $\Phi$.

We will now study the subleading operators $\Phi_{k}(k=1,2, \cdots)$ in the $\Psi \Psi$ OPE and show that they coincide with the following special descendants of the primary operator $\Phi$,

$$
\Phi^{(k)}=\left(\partial_{x}^{2}-4 \partial_{t}\right)^{k} \Phi
$$

The corresponding special descendants of $\bar{\Phi}$ are

$$
\bar{\Phi}^{(k)}=\left(\partial_{x}^{2}+4 \partial_{t}\right)^{k} \bar{\Phi}
$$

Thus the $\Psi \Psi$ OPE involves only one primary operator $\Phi$. This will be shown in the following steps. Firstly, in section 4.2.1, we fix the form of the special descendants $\Phi^{(k)}$ appearing in the OPE by studying a part of the decomposition of the four-point function (4.2). Next, we compute the coefficients of the $\Psi \Psi$ OPE involving the $\Phi^{(k)}$ 's. Finally, we show that there are no subleading operators other than $\Phi^{(k)}$ appearing in the $\Psi \Psi$ OPE. (For example, a primary operator $\Phi^{\prime}$ with dimension $\frac{3}{2}+\nu+2 n$, where $n$ is a positive integer, could appear on the r.h.s. of (4.4). We have to exclude this type of possibilities.) This is done in section 4.2 .2 by completely reproducing the full pairwise equal-time four-point function (3.13) just by summing up contributions from the primary operator $\Phi$ together with $\Phi^{(k)}$. This shows in particular that the $\Psi \Psi$ OPE is exhausted by the primary operator $\Phi$ and its special descendants $\Phi^{(k)}$. (In other words, one can put $\Phi_{k}=\Phi^{(k)}$ in (4.4).)

Throughout section 4.2 we will assume $x_{21}>0, x_{43}>0$ without loss of generality. Under this assumption, all formulae are valid for both the bosonic and fermionic theories.

\subsubsection{The contribution from the descendants of $\bar{\Phi}, \Phi$}

We will fix the descendants of $\Phi, \Phi$ appearing in the OPE (4.3) and (4.4). We will see that the following observation is essential: each term in the "s-channel" decomposition (4.2) of the four-point function contains $X$ only in the exponent and not in the prefactor of the exponential factor $e^{-\frac{X^{2}}{t}}$.

We consider a part of the "s-channel" decomposition (4.2), namely, the leading order terms in the expansion in terms of $x_{21}$ (keeping all subleading terms in the expansion by $\left.x_{43}\right)$,

$$
\begin{aligned}
\langle\Psi(t, & \left.\left.x_{4}\right) \Psi\left(t, x_{3}\right) \bar{\Psi}\left(0, x_{2}\right) \bar{\Psi}\left(0, x_{1}\right)\right\rangle \\
& \approx e^{-\frac{x_{43}^{2}}{4 t}} e^{-\frac{x^{2}}{t}} \times \sqrt{\frac{x_{21} x_{43}}{4 \pi t^{3}}}\left(\frac{x_{21} x_{43}}{4 t}\right)^{\nu} \frac{1}{\Gamma(\nu+1)} \\
& =\sum_{k=0}^{+\infty} \frac{1}{k !}\left(-\frac{x_{43}^{2}}{4 t}\right)^{k} e^{-\frac{x^{2}}{t}} \times \sqrt{\frac{x_{21} x_{43}}{4 \pi t^{3}}}\left(\frac{x_{21} x_{43}}{4 t}\right)^{\nu} \frac{1}{\Gamma(\nu+1)} .
\end{aligned}
$$

These terms should arise from the lowest dimension operator $\bar{\Phi}$ in the $\bar{\Psi}\left(0, x_{2}\right) \bar{\Psi}\left(0, x_{1}\right)$ OPE. Each term in this series corresponds to each operator contained in the $\Psi\left(t, x_{4}\right) \Psi\left(t, x_{3}\right)$ OPE. Now, in a theory with $z=2$ Schrödinger symmetry, primary operators with different scaling dimensions have vanishing two-point functions [32]. This means 
that $k \geq 1$ terms in (4.48) must all come from the descendants of $\Phi$ appearing in the $\Psi \Psi$ OPE.

In order to obtain the expression for these descendant operators, we need to know the two-point functions between a primary operator and its descendants. We will set $\left(x_{1}+x_{2}\right) / 2=0,\left(x_{3}+x_{4}\right) / 2=X$, for simplicity. Let us first consider the $k=1$ case. The relevant descendant operators should have dimension $\Delta_{0}+2$; they are $\partial_{x}^{2} \Phi$ and $\partial_{t} \Phi$. (We recall that $\Delta_{0}=\frac{3}{2}+\nu$.) Taking spacetime derivatives of the two-point function (4.9), we obtain

$$
\begin{aligned}
\left\langle\partial_{x}^{2} \Phi(t, X) \bar{\Phi}(0,0)\right\rangle & =\frac{1}{t^{\Delta_{0}}}\left(-\frac{2}{t}+\frac{4 X^{2}}{t^{2}}\right) e^{-\frac{X^{2}}{t}} \\
\left\langle\partial_{t} \Phi(t, X) \Phi(0,0)\right\rangle & =\frac{1}{t^{\Delta_{0}}}\left(-\frac{\Delta_{0}}{t}+\frac{X^{2}}{t^{2}}\right) e^{-\frac{X^{2}}{t}}
\end{aligned}
$$

Notice that each of the expressions contains $X$ in the prefactor of $e^{-\frac{X^{2}}{t}}$. However, we see that the $k=1$ term (in fact, all terms) in (4.48) does not contain $X$ in the prefactor of $e^{-\frac{X^{2}}{t}}$. Therefore, the special linear combination of the descendant operators $\partial_{x}^{2} \Phi$ and $\partial_{t} \Phi$,

$$
\Phi^{(1)}=\left(\partial_{x}^{2}-4 \partial_{t}\right) \Phi
$$

must be responsible for the $k=1$ term in (4.48). The linear combination $\Phi^{(1)}$ is constructed so that the two-point function

$$
\left\langle\Phi^{(1)}(t, X) \bar{\Phi}(0,0)\right\rangle=4\left(\Delta_{0}-\frac{1}{2}\right) \times \frac{1}{t^{\Delta_{0}+1}} e^{-\frac{X^{2}}{t^{2}}},
$$

does not contain $X$ in the prefactor of $e^{-\frac{X^{2}}{t}}$. Thus, the first subleading term in the $\Psi \Psi$ OPE should contain descendants of $\Phi$ only in the form of $\Phi^{(1)}$.

One can repeat this process of forming linear combinations of descendant operators further to construct special descendant operators $\Phi^{(k)}$; we observe that the necessary computations are the same, except that $\Delta_{0}$ should be replaced by $\Delta_{0}+1$ and then by $\Delta_{0}+2$, and so forth. ${ }^{23}$ We obtain the special descendant operators

$$
\Phi^{(k)}=\left(\partial_{x}^{2}-4 \partial_{t}\right)^{k} \Phi,
$$

with the two-point functions

$$
\begin{aligned}
\left\langle\left(\partial_{x}^{2}-4 \partial_{t}\right)^{k} \Phi(t, X) \bar{\Phi}(0,0)\right\rangle & =4^{k}\left(\Delta_{0}-\frac{1}{2}+k-1\right) \cdots\left(\Delta_{0}-\frac{1}{2}\right) \frac{1}{t^{\Delta_{0}+k}} e^{-\frac{X^{2}}{t}} \\
& =4^{k} \frac{\Gamma(\nu+k+1)}{\Gamma(\nu+1)} \frac{1}{t^{\frac{3}{2}+\nu+k}} e^{-\frac{X^{2}}{t}}
\end{aligned}
$$

which do not contain $X$ in the prefactor of $e^{-\frac{X^{2}}{t}}$. (We used $\Delta_{0}=\frac{3}{2}+\nu$ above.)

\footnotetext{
${ }^{23}$ To construct the special descendant operators by linear combinations, the operators arising at each step by applying $\partial_{x}^{2}$ and $\partial_{t}$ should be linearly independent. This is assured for $\Delta_{0}>\frac{1}{2}$.
} 
The coefficients before $\Phi^{(k)}$ in the $\Psi \Psi$ OPE can be read off from (4.48) using (4.54) and (4.8). We obtain,

$$
\begin{aligned}
& \Psi\left(t, x_{4}\right) \Psi\left(t, x_{3}\right) \\
= & \sum_{k=0}^{+\infty} \frac{1}{2^{\nu}(4 \pi)^{\frac{1}{4}} \sqrt{\Gamma(\nu+1)}} \times(-1)^{k} \frac{1}{k !} \frac{1}{4^{2 k}} \frac{\Gamma(\nu+1)}{\Gamma(\nu+k+1)} \times x_{43}^{\nu+\frac{1}{2}+2 k} \times \Phi^{(k)}\left(t, \frac{x_{3}+x_{4}}{2}\right) .
\end{aligned}
$$

The term with $k=0$ of course is the leading order term in the OPE we have already seen in (4.3) and (4.8), $\Phi=\Phi^{(0)}$.

We have shown that the descendants of $\Phi$ should appear in the $\Psi \Psi$ OPE in the way given in (4.55). However, there could be another primary operator, say, $\Phi^{\prime}$ with dimension $\Delta_{0}+2 n$ where $n$ is a non-negative integer, which enters the $\Psi \Psi$ OPE together with its descendants. (In other words, $\Phi_{k}$ in (4.4) may be a linear combination of $\Phi^{(k)}$ and $\Phi^{\prime}$ itself or its descendants.) We will exclude this possibility in section 4.2.2. Once this is done, we can conclude that (4.55) is complete and coincides with (4.4) with $\Phi_{k}=\Phi^{(k)}$ and

$$
C_{k}=\frac{1}{2^{\nu}(4 \pi)^{\frac{1}{4}} \sqrt{\Gamma(\nu+1)}} \times(-1)^{k} \frac{1}{k !} \frac{1}{4^{2 k}} \frac{\Gamma(\nu+1)}{\Gamma(\nu+k+1)} .
$$

By repeating the same argument starting from the leading order terms in $x_{43}$ of (4.2), we obtain similar results for the $\bar{\Psi} \bar{\Psi}$ OPE. Thus, descendants of $\bar{\Phi}$ must enter the $\bar{\Psi} \bar{\Psi}$ OPE in the following special linear combinations, ${ }^{24}$

$$
\bar{\Phi}^{(k)}=\left(\partial_{x}^{2}+4 \partial_{t}\right)^{k} \bar{\Phi}
$$

which are constructed so that the two-point functions

$$
\left\langle\left(\partial_{x}^{2}+4 \partial_{t}\right)^{k} \bar{\Phi}(t, X) \Phi(0,0)\right\rangle=4^{k} \frac{\Gamma(\nu+k+1)}{\Gamma(\nu+1)} \frac{1}{t^{\frac{3}{2}+\nu+k}} e^{-\frac{X^{2}}{t}},
$$

do not contain $X$ in the prefactor of $e^{-\frac{X^{2}}{t}}$. The $\bar{\Psi} \overline{\text { OPE becomes }}$

$$
\begin{aligned}
& \bar{\Psi}\left(0, x_{2}\right) \bar{\Psi}\left(0, x_{1}\right) \\
= & \sum_{n=0}^{+\infty} \frac{1}{2^{\nu}(4 \pi)^{\frac{1}{4}} \sqrt{\Gamma(\nu+1)}} \times(-1)^{n} \frac{1}{n !} \frac{1}{4^{2 n}} \frac{\Gamma(\nu+1)}{\Gamma(\nu+n+1)} \times x_{21}^{\nu+\frac{1}{2}+2 n} \times \bar{\Phi}^{(n)}\left(0, \frac{x_{1}+x_{2}}{2}\right) .
\end{aligned}
$$

Again we will see in section 4.2.2 that (4.59) is complete and coincides with (4.3) with $\bar{\Phi}_{k}=\bar{\Phi}^{(k)}$ and (4.56).

The important property of the special descendants $\bar{\Phi}^{(m)}, \Phi^{(n)}$ is that their mutual two-point functions

$$
\left\langle\bar{\Phi}^{(m)}(t, X) \Phi^{(n)}(0,0)\right\rangle=4^{m+n} \frac{\Gamma(\nu+m+n+1)}{\Gamma(\nu+1)} \frac{1}{t^{\frac{3}{2}+\nu+m+n}} e^{-\frac{X^{2}}{t}},
$$

\footnotetext{
${ }^{24}$ We note that the sign flip before $\partial_{t}$ of $\bar{\Phi}^{(k)}$ compared to $\Phi^{(k)}$ is due to our use of Euclidean time, $\Phi(t, x)=$ $e^{H t} \Phi(0, x) e^{-H t}, \bar{\Phi}(t, x)=e^{H t} \bar{\Phi}(0, x) e^{-H t}$. Thus we have $\overline{\Phi^{(k)}(0, x)}=\bar{\Phi}^{(k)}(0, x)$ because $\overline{\partial_{t} \Phi(0, x)}=$ $\overline{[H, \Phi(0, x)]}=-[H, \bar{\Phi}(0, x)]=-\partial_{t} \bar{\Phi}(0, x)$.
} 
do not contain $X$ in the prefactor of $e^{-\frac{X^{2}}{t}}$. This reflects the absence of $X$ in the prefactor of $e^{-\frac{X^{2}}{t}}$ for all terms contained in (4.2).

\subsubsection{Reproducing full four-point function from OPE}

Here we shall prove that the OPEs (4.55) and (4.59) are complete by showing that they fully reproduce the pairwise equal-time four-point function (3.13).

From the OPEs (4.55) and (4.59) and the two-point functions (4.60), we obtain

$$
\begin{aligned}
\left\langle\Psi\left(t, x_{4}\right)\right. & \left.\Psi\left(t, x_{3}\right) \bar{\Psi}\left(0, x_{2}\right) \bar{\Psi}\left(0, x_{1}\right)\right\rangle \\
= & \frac{1}{4^{\nu}(4 \pi)^{\frac{1}{2}} \Gamma(\nu+1)} \\
& \times \sum_{m=0}^{\infty} \sum_{n=0}^{\infty} \frac{(-1)^{n}}{n !} \frac{1}{4^{2 n}} \frac{\Gamma(\nu+1)}{\Gamma(\nu+n+1)} x_{43}^{\nu+\frac{1}{2}+2 n} \times \frac{(-1)^{m}}{m !} \frac{1}{4^{2 m}} \frac{\Gamma(\nu+1)}{\Gamma(\nu+m+1)} x_{21}^{\nu+\frac{1}{2}+2 m} \\
& \times 4^{m+n} \frac{\Gamma(\nu+m+n+1)}{\Gamma(\nu+1)} \frac{1}{t^{\frac{3}{2}+\nu+m+n}} e^{-\frac{X^{2}}{t}} \\
= & e^{-\frac{X^{2}}{t}} \sqrt{\frac{x_{21} x_{43}}{4 \pi t^{3}}}\left(\frac{x_{21} x_{43}}{4 t}\right)^{\nu} \sum_{m=0}^{\infty} \sum_{n=0}^{\infty} \frac{(-1)^{m+n}}{m ! n !} \frac{\Gamma(\nu+m+n+1)}{\Gamma(\nu+m+1) \Gamma(\nu+n+1)} \frac{x_{21}^{2 m} x_{43}^{2 n}}{(4 t)^{m+n}}
\end{aligned}
$$

We wish to show this formula agrees with the "s-channel" decomposition (4.2). Factoring out the common factor, the identity we have to show is

$$
\sum_{m=0}^{\infty} \sum_{n=0}^{\infty} \frac{(-1)^{m+n}}{m ! n !} \frac{\Gamma(\nu+m+n+1)}{\Gamma(\nu+m+1) \Gamma(\nu+n+1)} \frac{x_{21}^{2 m} x_{43}^{2 n}}{(4 t)^{m+n}}=e^{-\frac{x_{21}^{2}+x_{43}^{2}}{4 t}} \sum_{k=0}^{\infty} \frac{\left(\frac{x_{21} x_{43}}{4 t}\right)^{2 k}}{k ! \Gamma(\nu+k+1)},
$$

which is equivalent to

$$
\frac{1}{m ! n !} \frac{\Gamma(\nu+m+n+1)}{\Gamma(\nu+m+1) \Gamma(\nu+n+1)}=\sum_{k=0}^{\min (m, n)} \frac{1}{k ! \Gamma(\nu+k+1)} \frac{1}{(m-k) !} \frac{1}{(n-k) !} .
$$

Now, using the Pochhammer symbol, we have

$$
\begin{aligned}
\text { (r.h.s.) } & =\sum_{k=0}^{\min (m, n)} \frac{1}{k !} \frac{1}{(\nu+1)_{k} \Gamma(\nu+1)}(-1)^{k} \frac{(-m)_{k}}{m !}(-1)^{k} \frac{(-n)_{k}}{n !} \\
& =\sum_{k=0}^{+\infty} \frac{1}{k !} \frac{1}{(\nu+1)_{k} \Gamma(\nu+1)} \frac{(-m)_{k}}{m !} \frac{(-n)_{k}}{n !} \\
& =\frac{1}{\Gamma(\nu+1) m ! n !}{ }_{2} F_{1}(-m,-n ; \nu+1 ; 1)=(\text { l.h.s. }),
\end{aligned}
$$

where we used a well-known identity for hypergeometric functions,

$$
{ }_{2} F_{1}(a, b ; c ; 1)=\frac{\Gamma(c) \Gamma(c-a-b)}{\Gamma(c-a) \Gamma(c-b)} .
$$

Thus the OPEs (4.55) and (4.59) reproduce the pairwise equal-time four-point function (3.13) fully and hence are complete. 


\subsection{Three-point function $\langle\Psi \Psi \bar{\Phi}\rangle$}

We have seen in section 4.2 that there is only one primary operator, $\bar{\Phi}$, involved in the OPE $\bar{\Psi}(x) \bar{\Psi}(0)$. By pinching the two insertion points of $\bar{\Psi}$ of the four-point functions obtained in section 3, we can compute the three-point function $\langle\Psi \Psi \bar{\Phi}\rangle$.

In [52], Golkar and Son showed that the constraint from Schrödinger symmetry alone fixes the spacetime dependence of the three-point function (except, of course, the overall coefficient which contains the dynamical information of the theory considered) when one of the operators involved saturates the unitarity bound, which, in one space dimension, is $\Delta \geq \frac{1}{2}$. The field $\Psi$ saturates the unitarity bound. The form of the three-point function we obtained is consistent with Golkar and Son's analysis. Since their analysis is done in Minkowski signature, and the continuation to Euclidean signature is not entirely trivial, we give the analysis done for theories with Euclidean time in appendix D. The appendix also contains a discussion of the boundary conditions necessary to fix the spacetime dependence. We point out that the boundary conditions give different constraints for one space dimension compared to other cases.

\subsubsection{Three-point functions with two operators at equal-time}

First, we consider the case in which two $\Psi$ 's are inserted at the same time,

$$
\left\langle\Psi\left(t, x_{3}\right) \Psi\left(t, x_{2}\right) \bar{\Phi}(0,0)\right\rangle \text {. }
$$

We will consider the case $t>0$; if $t<0$ the three-point function vanishes trivially since the operator $\Psi(x)$ annihilates the vacuum.

We keep the leading order term in the expansion in $x_{21}=x_{2}-x_{1}$ in the pairwise equal-time four-point function (3.13) using (4.1). Putting $\frac{x_{1}+x_{2}}{2}=0$, we obtain

$$
\begin{aligned}
& \left\langle\Psi\left(t, x_{4}\right) \Psi\left(t, x_{3}\right) \bar{\Psi}\left(0, x_{2}\right) \bar{\Psi}\left(0, x_{1}\right)\right\rangle \\
& \quad \approx e^{-\frac{x_{43}^{2}+\left(x_{3}+x_{4}\right)^{2}}{4 t}} \times \sqrt{\frac{x_{21} x_{43}}{4 \pi t^{3}}} \frac{1}{\Gamma(\nu+1)}\left(\frac{x_{21} x_{43}}{4 t}\right)^{\nu}+\cdots .
\end{aligned}
$$

This is valid when $x_{43}>0, x_{21}>0$ for both the bosonic and fermionic models. Comparing this with the leading term of the OPE (4.59)

$$
\bar{\Psi}\left(0, x_{2}\right) \bar{\Psi}\left(0, x_{1}\right) \approx \frac{1}{2^{\nu}(4 \pi)^{\frac{1}{4}} \sqrt{\Gamma(\nu+1)}} x_{21}^{\nu+\frac{1}{2}} \times \bar{\Phi}(0,0),
$$

we obtain, after relabelling,

$$
\begin{aligned}
& \left\langle\Psi\left(t, x_{3}\right) \Psi\left(t, x_{2}\right) \bar{\Phi}(0,0)\right\rangle \\
& \quad=\frac{1}{2^{\nu}(4 \pi)^{\frac{1}{4}} \sqrt{\Gamma(\nu+1)}} \times x_{32}^{\nu+\frac{1}{2}} t^{-\left(\nu+\frac{3}{2}\right)} \times e^{-\frac{x_{3}^{2}+x_{2}^{2}}{2 t}} .
\end{aligned}
$$

This expression is valid when $x_{32}>0$ for both the bosonic and fermionic models. The formula valid for $x_{32}<0$ can be obtained easily as is done for the equal-time four-point function, (3.16) and (3.17). The normalisation conditions for the operators $\Psi$ and $\Phi$ are fixed by the two-point functions, (2.9) and (4.9). 
The result (4.69) is, apart from an overall factor, the product of two free propagators $\left\langle\Psi\left(t, x_{3}\right) \bar{\Psi}(0,0)\right\rangle\left\langle\Psi\left(t, x_{2}\right) \bar{\Psi}(0,0)\right\rangle \sim \frac{1}{t} e^{-\frac{x_{3}^{2}+x_{2}^{2}}{2 t}}$ dressed with a factor (depending on $x_{32}$ and t) $x_{32}^{\nu+\frac{1}{2}} t^{-\left(\nu+\frac{1}{2}\right)}$.

\subsubsection{Three-point functions in general position}

Integral representation. We consider the three-point function in general position,

$$
\left\langle\Psi\left(t_{3}, x_{3}\right) \Psi\left(t_{2}, x_{2}\right) \bar{\Phi}\left(t_{1}, x_{1}\right)\right\rangle
$$

Here we will only consider the bosonic model. We set $x_{1}=0, t_{1}=0$ using translational invariance. We assume $t_{3}>t_{2}$ without loss of generality. We consider the case $t_{2}>t_{1}$ since otherwise the three-point function vanishes trivially, the operator $\Psi\left(t_{2}, x_{2}\right)$ annihilating the vacuum.

We begin with the integral representation (3.22) for the four-point function $\left\langle\Psi\left(t_{4}, x_{4}\right) \Psi\left(t_{3}, x_{3}\right) \bar{\Psi}\left(0, x_{2}\right) \bar{\Psi}\left(0, x_{1}\right)\right\rangle$, with $t_{4}>t_{3}>0$. (The labels 3,4 will be replaced respectively by 2,3 later.) We consider the limit $x_{2} \rightarrow x_{1}$ and keep the leading order term in the expansion in terms of $x_{21}$. Writing $t_{3}=t, t_{43}=t^{\prime}$ and setting $\frac{x_{1}+x_{2}}{2}=0$, we obtain, using the leading order term of (4.1) and (3.16),

$$
\begin{aligned}
& \left\langle\Psi\left(t+t^{\prime}, x_{4}\right) \Psi\left(t, x_{3}\right) \bar{\Psi}\left(0, x_{2}\right) \bar{\Psi}\left(0, x_{1}\right)\right\rangle \\
\approx & \int e^{-\frac{x_{4^{\prime} 3}^{2}+\left(x_{3}+x_{4^{\prime}}\right)^{2}}{4 t}} \times \sqrt{\frac{\left|x_{21} x_{4^{\prime} 3}\right|}{4 \pi t^{3}}} \times\left|\frac{x_{21} x_{4^{\prime} 3}}{4 t}\right|^{\nu} \frac{1}{\Gamma(\nu+1)} \times \sqrt{\frac{1}{2 \pi t^{\prime}}} e^{-\frac{1}{2} \frac{x_{4 \prime^{\prime}}^{2}}{t^{\prime}}} d x_{4}^{\prime} .
\end{aligned}
$$

Note that we used (3.16) valid for the bosonic model and applicable for both positive and negative $x_{4^{\prime} 3}$.

Comparing (4.71) with the leading order term in the OPE (4.59),

$$
\bar{\Psi}\left(0, x_{2}\right) \bar{\Psi}\left(0, x_{1}\right) \approx \frac{1}{2^{\nu}(4 \pi)^{\frac{1}{4}} \sqrt{\Gamma(\nu+1)}}\left|x_{21}\right|^{\nu+\frac{1}{2}} \times \bar{\Phi}(0,0),
$$

we obtain an integral representation of the three-point function in general position,

$$
\begin{aligned}
& \left\langle\Psi\left(t+t^{\prime}, x_{4}\right) \Psi\left(t, x_{3}\right) \Phi(0,0)\right\rangle \\
& \quad=\frac{1}{2^{\nu+1} \pi^{\frac{3}{4}} \sqrt{\Gamma(\nu+1)}} \frac{1}{\sqrt{t^{\prime}}} \frac{1}{t^{\frac{3}{2}+\nu}} \int e^{-\frac{x_{4^{\prime} 3}^{2}+\left(x_{4^{\prime}}+x_{3}\right)^{2}}{4 t}} \times\left|x_{4^{\prime} 3}\right|^{\nu+\frac{1}{2}} \times e^{-\frac{1}{2} \frac{x_{44^{\prime}}^{2}}{t^{\prime}}} d x_{4^{\prime}} .
\end{aligned}
$$

Three-point function in general position. This integral can be worked out, separating contributions from $x_{4^{\prime} 3}>0$ and $x_{4^{\prime} 3}<0$. The result can be expressed in terms of the parabolic cylinder functions or the confluent hypergeometric functions. The details of the computation, including the comparison to the generic form of the three-point function found by Henkel $[32,33]$, are given in appendix C. The final result expressed via the 
confluent hypergeometric function $M(a, b, z)$ (in the notation of [78]) is,

$$
\begin{aligned}
& \left\langle\Psi\left(t_{3}, x_{3}\right) \Psi\left(t_{2}, x_{2}\right) \bar{\Phi}\left(t_{1}, x_{1}\right)\right\rangle \\
& =\frac{\Gamma\left(\nu+\frac{3}{2}\right)}{2^{\frac{3}{2} \nu+\frac{3}{4}} \pi^{\frac{1}{4}} \sqrt{\Gamma(\nu+1)} \Gamma\left(\frac{\nu}{2}+\frac{5}{4}\right)} \sqrt{\frac{t_{32}^{\nu+\frac{1}{2}}}{t_{31}^{\nu+\frac{3}{2}} t_{21}^{\nu+\frac{3}{2}}}} e^{-\frac{x_{21}^{2}}{2 t_{21}} e^{-\frac{x_{31}^{2}}{2 t_{31}}}} \\
& \quad \times e^{-\frac{w^{2}}{2}} M\left(\frac{\nu}{2}+\frac{3}{4}, \frac{1}{2}, \frac{1}{2} w^{2}\right) .
\end{aligned}
$$

where $w$ is a quantity which is invariant under the Schrödinger symmetry,

$$
w=\left(\frac{x_{21}}{t_{21}}-\frac{x_{32}}{t_{32}}\right) \sqrt{\frac{t_{21} t_{32}}{t_{31}}} .
$$

We have chosen $t_{3}>t_{2}>t_{1}$ and hence $w \in \mathbb{R}$. (In the notation of section $3.3, \frac{1}{2} w^{2}=v$ ).

The spacetime dependence is consistent with the analysis based on the Schrödinger symmetry by Golkar and Son [52]. See appendix D for a detailed comparison.

By taking the limit $t_{32} \rightarrow 0$ of (4.74), we recover the result of section 4.3.1. See appendix C.5. The special case $\nu=-\frac{1}{2}$ agrees with the result for the free-boson. See appendix E.2.

\subsection{Two-point function of the charge-zero operators appearing in the " $t$ - channel" decomposition}

In this subsection, we will deduce a peculiar property of the charge-zero operators $\mathcal{J}_{m}$ $(m=1,2, \cdots)$ appearing in the $\bar{\Psi} \Psi$ OPE. Namely, we will show that the two-point functions

$$
\left\langle\mathcal{J}_{m}\left(t_{1}, x_{1}\right) \mathcal{J}_{n}\left(t_{2}, x_{2}\right)\right\rangle \quad(m>0, n>0)
$$

vanish if $t_{1} \neq t_{2}$. Note that the two-point functions are non-vanishing and finite in general for $t_{1}=t_{2},(4.23)$. Our argument is fairly general and is not restricted to the Calogero model. The assumptions are the existence of the OPE, the scale invariance, the U(1) symmetry, and the uniqueness of the vacuum. Hence the argument will apply in particular to any theory with Schrödinger symmetry and with a unique vacuum.

The basis of the argument is the following property of the four-point function $\left\langle\Psi\left(t_{4}, x_{4}\right) \Psi\left(t_{3}, x_{3}\right) \bar{\Psi}\left(t_{2}, x_{2}\right) \bar{\Psi}\left(t_{1}, x_{1}\right)\right\rangle$. Depending on the time-order of the operators, the four-point function (i) has a nontrivial form, (ii) factorises into a product of two-point functions, or (iii) vanishes. The first possibility occurs when the time-ordered product of the operators has the form $\Psi \Psi \bar{\Psi} \bar{\Psi}$, i.e. when $t_{4}>t_{1}, t_{4}>t_{2}, t_{3}>t_{2}$, and $t_{3}>t_{1}$ hold. The pairwise equal-time four-point function derived in section 3.1 is a particular case of this possibility. The second possibility occurs when the time-ordered product has the form $\Psi \bar{\Psi} \Psi \bar{\Psi}$, i.e. when $t_{4}>t_{2}>t_{3}>t_{1}, t_{3}>t_{2}>t_{4}>t_{1}, t_{4}>t_{1}>t_{3}>t_{2}$, or $t_{3}>t_{1}>t_{4}>t_{2}$ hold. The third possibility occurs when the operator with the smallest time is $\Psi$ or the largest time is $\bar{\Psi}$ (because of $\Psi|0\rangle=0$ and $\langle 0| \bar{\Psi}=0$ ). 
Let us consider the second possibility; to be specific, we focus on the case $t_{4}>t_{2}>$ $t_{3}>t_{1}$. Then we have

$$
\begin{aligned}
\left\langle\Psi \left( t_{4},\right.\right. & \left.\left.x_{4}\right) \Psi\left(t_{3}, x_{3}\right) \bar{\Psi}\left(t_{2}, x_{2}\right) \bar{\Psi}\left(t_{1}, x_{1}\right)\right\rangle \\
& =\left\langle 0\left|T \Psi\left(t_{4}, x_{4}\right) \Psi\left(t_{3}, x_{3}\right) \bar{\Psi}\left(t_{2}, x_{2}\right) \bar{\Psi}\left(t_{1}, x_{1}\right)\right| 0\right\rangle \\
& =\left\langle 0\left|\Psi\left(t_{4}, x_{4}\right) \bar{\Psi}\left(t_{2}, x_{2}\right) \Psi\left(t_{3}, x_{3}\right) \bar{\Psi}\left(t_{1}, x_{1}\right)\right| 0\right\rangle \\
& =\left\langle 0\left|\Psi\left(t_{4}, x_{4}\right) \bar{\Psi}\left(t_{2}, x_{2}\right)\right| 0\right\rangle\left\langle 0\left|\Psi\left(t_{3}, x_{3}\right) \bar{\Psi}\left(t_{1}, x_{1}\right)\right| 0\right\rangle \\
& =\left\langle\Psi\left(t_{4}, x_{4}\right) \bar{\Psi}\left(t_{2}, x_{2}\right)\right\rangle\left\langle\Psi\left(t_{3}, x_{3}\right) \bar{\Psi}\left(t_{1}, x_{1}\right)\right\rangle .
\end{aligned}
$$

In going from the third to the fourth line, we inserted a complete set of eigenstates between $\bar{\Psi}\left(t_{2}, x_{2}\right)$ and $\Psi\left(t_{3}, x_{3}\right)$. Then we used the fact that the state $\Psi\left(t_{3}, x_{3}\right) \bar{\Psi}\left(t_{1}, x_{1}\right)|0\rangle$ has vanishing $U(1)$ charge and hence should coincide with the vacuum $|0\rangle$ up to a constant factor.

Let us consider the limit where both pairs of spacetime points $(4,2)$ and $(3,1)$ become coincident. (More precisely, the limit $x_{31} \rightarrow 0, t_{31} \rightarrow 0$ with fixed $\frac{x_{31}^{2}}{t_{31}}$, and the similar coincident limit for the points $(4,2)$ should be taken.) In this limit, we can use the OPE $(4.22)$ of $\Psi\left(t_{4}, x_{4}\right) \bar{\Psi}\left(t_{2}, x_{2}\right)$ and of $\Psi\left(t_{3}, x_{3}\right) \bar{\Psi}\left(t_{1}, x_{1}\right)$ which we have studied in section 4.1.2,

$$
\Psi(t, x) \bar{\Psi}(0,0)=\sum_{k=0}^{\infty} x^{k-1} f_{k}\left(\frac{x^{2}}{t}\right) \mathcal{J}_{k}\left(\frac{t}{2}, \frac{x}{2}\right) .
$$

We recall that $\mathcal{J}_{0}$ is the identity operator and

$$
x^{-1} f_{0}\left(\frac{x^{2}}{t}\right)=\frac{1}{\sqrt{2 \pi t}} e^{-\frac{x^{2}}{2 t}}=\langle\Psi(t, x) \bar{\Psi}(0,0)\rangle .
$$

Because of the factorisation property (4.77), the four-point function depends on $\left(t_{31}, x_{31}\right)$ and $\left(t_{42}, x_{42}\right)$ but not on the relative position between $\left(t_{2}, x_{2}\right) \approx\left(t_{4}, x_{4}\right)$ and $\left(t_{1}, x_{1}\right) \approx$ $\left(t_{3}, x_{3}\right)$. This implies the vanishing of the two-point functions,

$$
\left\langle\mathcal{J}_{m}\left(t_{2}, x_{2}\right) \mathcal{J}_{n}\left(t_{1}, x_{1}\right)\right\rangle=0,
$$

for $t_{2}>t_{1}$, except for the case when both the operators are the identity operator $(m=$ $0, n=0)$. Indeed, the identity operator appearing in the $\Psi \bar{\Psi}$ OPE completely reproduces the factorised four-point function.

The situation is quite different from that considered in section 4.1.2, where we need infinitely many nonzero equal-time two-point functions $\left\langle\mathcal{J}_{m}(t, x) \mathcal{J}_{n}\left(t, x^{\prime}\right)\right\rangle=\frac{D_{m n}}{\left(x-x^{\prime}\right)^{m+n}}$, in order to reproduce the pairwise equal-time four-point function (except for the special cases where $\nu$ is a half odd integer).

We can make this argument more precise by considering three-point functions $\left\langle\Psi \mathcal{J}_{k} \bar{\Psi}\right\rangle$. We start from the non-factorised four-point function, $t_{4}>t_{3}>t_{2}>t_{1}$. We then take the coincident limit of the spacetime points $(3,2)$ and use the OPE $\bar{\Psi}\left(t_{3}, x_{3}\right) \Psi\left(t_{2}, x_{2}\right)$. Focusing on each term in the OPE expansion, one obtains the three-point functions where $\bar{\Psi}$ is inserted at the spacetime point $4, \mathcal{J}_{k}$ is inserted at $3=2$, and $\Psi$ at 1 .

Now, if we start with a different time-ordering, say, $t_{3}>t_{2}>t_{4}>t_{1},{ }^{25}$ the factorisation (4.77) implies that the three-point function vanishes except for the special case where

\footnotetext{
${ }^{25}$ Note that the points 3 and 2 should be adjacent in the time-ordering in order that one can take the coincident limit.
} 
the operator appearing from the $\Psi \bar{\Psi}$ OPE is the identity operator. Thus, the three-point function

$$
\left\langle\Psi\left(t_{3}, x_{3}\right) \mathcal{J}_{m}\left(t_{2}, x_{2}\right) \bar{\Psi}\left(t_{1}, x_{1}\right)\right\rangle \quad(m=1,2, \cdots)
$$

is non-vanishing only when $t_{3}>t_{2}>t_{1}$.

We then take the coincident limit of the points 3 and 1 . Unless we maintain the timeordering $t_{3}>t_{2}>t_{1}$ during the coincident limit, the result vanishes (when $\mathcal{J}_{m}$ is not an identity operator). We again reach the conclusion that the two-point function

$$
\left\langle\mathcal{J}_{m}\left(t_{1}, x_{1}\right) \mathcal{J}_{n}\left(t_{2}, x_{2}\right)\right\rangle \quad(m>0, n>0)
$$

can have a nonzero value only if $t_{1}=t_{2}$.

The vanishing of the two-point functions can be deduced from the following more formal argument. One may rewrite the two-point function as the vacuum expectation value of the time-ordered product,

$$
\left\langle\mathcal{J}_{m}\left(t_{1}, x_{1}\right) \mathcal{J}_{n}\left(t_{2}, x_{2}\right)\right\rangle=\left\langle 0\left|T \mathcal{J}_{m}\left(t_{1}, x_{1}\right) \mathcal{J}_{n}\left(t_{2}, x_{2}\right)\right| 0\right\rangle .
$$

Assume that $t_{1}>t_{2}$. Then we have

$$
\left\langle\mathcal{J}_{m}\left(t_{1}, x_{1}\right) \mathcal{J}_{n}\left(t_{2}, x_{2}\right)\right\rangle=\left\langle 0\left|\mathcal{J}_{m}\left(t_{1}, x_{1}\right) \mathcal{J}_{n}\left(t_{2}, x_{2}\right)\right| 0\right\rangle .
$$

We can insert a complete set of states between $\mathcal{J}_{m}\left(t_{1}, x_{1}\right)$ and $\mathcal{J}_{m}\left(t_{2}, x_{2}\right)$. Since $\mathcal{J}_{n}\left(t_{2}, x_{2}\right)|0\rangle$ has vanishing $\mathrm{U}(1)$ charge and the only state with zero charge is the vacuum, we obtain

$$
\left\langle\mathcal{J}_{m}\left(t_{1}, x_{1}\right) \mathcal{J}_{n}\left(t_{2}, x_{2}\right)\right\rangle=\left\langle 0\left|\mathcal{J}_{m}\left(t_{1}, x_{1}\right)\right| 0\right\rangle\left\langle 0\left|\mathcal{J}_{n}\left(t_{2}, x_{2}\right)\right| 0\right\rangle
$$

By the scale invariance, the one-point function of any operator should vanish, unless the operator is an identity operator. Therefore we see that the two-point function $\left\langle\mathcal{J}_{m}\left(t_{1}, x_{1}\right) \mathcal{J}_{n}\left(t_{2}, x_{2}\right)\right\rangle$ should vanish unless $m=0, n=0$. This argument illustrates the subtlety involved in the two-point function for the case $t_{1}=t_{2}$. In order to have the finite equal-time two-point functions (4.23), which are required by the "t-channel" decomposition of the nontrivial four-point function and the $\Psi \bar{\Psi}$ OPE discussed in section 4.1.2, one must conclude that (4.84) does not hold when $t_{1}=t_{2}$,

$$
\left\langle\mathcal{J}_{m}\left(t, x_{1}\right) \mathcal{J}_{n}\left(t, x_{2}\right)\right\rangle \neq\left\langle 0\left|\mathcal{J}_{m}\left(t, x_{1}\right) \mathcal{J}_{n}\left(t, x_{2}\right)\right| 0\right\rangle,
$$

as otherwise the equal-time two-point functions would also vanish by the same argument.

In this subsection, we deduced features of charge- 0 operators arising from the $\bar{\Psi} \Psi$ OPE. It is clearly important to pursue this direction further. For example, by using the four-point function for general positions derived in section 3.3, one should be able to compute the three-point function $\left\langle\Psi \mathcal{J}_{m} \bar{\Psi}\right\rangle$. This will in turn give us information about the $\mathcal{J}_{m} \bar{\Psi}$ OPE. This is important in understanding the nature of the operators $\mathcal{J}_{m}$. We expect them to include the energy-momentum tensor and the symmetry currents. The $\mathcal{J}_{m} \bar{\Psi}$ OPE should tell us what kind of symmetries, if any, are associated with the operators $\mathcal{J}_{m}$. 


\section{Conclusion and discussion}

In this paper, we have pointed out that the Calogero model considered as a quantum field theory in one space and one time dimension via the second quantisation is a tractable yet nontrivial example of $z=2$ anisotropic scale invariant theory. We obtained the expression of the four-point function of the elementary fields for the special pairwise equal-time case (3.13). The general four-point function can also be expressed either in terms of a double convolution integral (3.19) or of a generalised hypergeometric function (3.23).

We have obtained new insights into the $z=2$ theories, exploiting the exact expression of the four-point function. We decomposed it in two different ways (the "s-channel" and "t-channel" decompositions studied in sections 4.1.1 and 4.1.2), corresponding to two different ways of applying the OPE. In this way, we have verified the OPE associativity for the model in the case of the particular four-point function. The "t-channel" decomposition is asymptotic rather than convergent. The exponentially small corrections to the asymptotic series also can be interpreted using the OPE (section 4.1.3). The asymptotic nature is inherently connected to the presence of the terms behaving as $e^{-a x^{2} / t}$ in Schrödinger invariant theories. This makes us suspect that the asymptotic nature of the "t-channel" decomposition and the interpretation of the exponentially small correction terms by the OPE are universal features of Schrödinger invariant theories rather than being specific to our model.

Our analysis suggests the importance of the equal-time observables (e.g., the pairwise equal-time four-point function). They have particularly simple forms but yet contain interesting dynamical information of the model such as the scaling dimensions and the OPE coefficients depending on the coupling constant.

The "s-channel" decomposition turns out to involve only one primary operator (section 4.2). Thus we have obtained an analogue of the conformal block in isotropic theories. We may call it the "Schrödinger block". We have obtained a special case of the Schrödinger block (in two spacetime dimensions). It is special in that it is restricted to the four-point functions of operators with $\Delta=\frac{1}{2}$. The scaling dimension of the operator running in the intermediate channel can be controlled by tuning the coupling constant $\nu$. We hope this result may serve as a building block in the bootstrap program of $z=2$ Schrödinger invariant theories.

By taking a certain limit of the four-point function we have computed a three-point function (section 4.3) and have found peculiar properties of correlation functions involving certain charge-zero operators (section 4.4).

The reason we are able to uncover these new features is because our model allows us to explicitly compute the four-point function. Previously obtained exact results for genuine interacting Schrödinger invariant field theories are restricted, to our knowledge, to computations of three-point functions and the associated OPE. ${ }^{26}$ These include the exact computation of a three-point function [82] and OPEs [45] for the fermion at unitarity (and for a related bosonic theory) in general space dimensions. For the computation of the OPE in systems with contact interactions in one space and one time dimension(which

\footnotetext{
${ }^{26}$ For holographic computations, see for example [73].
} 
are generally not scale invariant), see, for example, [83] and references therein. For a review of computations of observables related to the three-point correlation functions with Schrödinger symmetry in statistical models, see [84].

Four-point function in general position. We have presented the exact expression (3.24) of the four-point function in general position using a generalised hypergeometric function with three variables. The three variables are quantities invariant under the Schrödinger symmetry and thus are the analogues of the cross-ratios in the standard CFT.

The exact expression is worth further investigation. Firstly, by studying a certain limit of the expression, we should get a better understanding of the "t-channel" OPE and hence of the important charge-zero operators.

Secondly, the generalised hypergeometric function should obey certain connection formulae, analogous to those satisfied by the ordinary Gaussian hypergeometric functions. The connection formulae relate different expansions of the function valid for different limits one can take in their arguments. These different limits should correspond to the various ways of decomposing the four-point function by the OPE. Hence, the connection formulae should be a rather direct manifestation of the OPE associativity. A good example which shows the relevance of the hypergeometric functions and their connection formulae in the conformal bootstrap program is the Liouville CFT. A four-point function in the Liouville CFT is written directly in terms of the Gaussian hypergeometric function of the cross-ratio, and a connection formula between the hypergeometric functions indeed represents the OPE associativity [85].

Finally, we have seen that Schrödinger invariant theories have an intricate structure: if looked at from a certain perspective they are described by functions analogous to the confluent hypergeometric function (which can be represented by an asymptotic series when its argument goes to infinity), and from another perspective, they are described by functions analogous to the hypergeometric function (which can be expanded everywhere, even including the point at infinity, and represented as a convergent series). On the one hand, the pairwise equal-time four-point function is given in terms of a modified Bessel function, which is a special case of the confluent hypergeometric function. Also, the three-point function obtained as a limit of the four-point function is written by a confluent hypergeometric function. On the other hand, if considered as a function of one of the Schrödinger invariant "cross-ratios" (3.26), $\tau=\frac{t_{21} t_{43}}{t_{31} t_{42}}$, the four-point function should have features analogous to the hypergeometric function, consistent with the SL(2,R) subgroup of the Schrödinger symmetry discussed in [54]. The expression of the four-point function via a generalised hypergeometric function should embody this mixed feature. Expressed as a multiple series of a certain set of combinations of the variables valid for certain limits, the series should be of hypergeometric type. When another set of combinations of its variables is used, the series should have degenerate parameters, and have properties closer to the confluent hypergeometric functions rather than the hypergeometric functions.

Analogy to 2D CFT and the sine-Gordon model. The model we have considered in this paper, the Calogero model in the second-quantised formulation, has features analogous to the compactified free-boson CFT in two spacetime dimensions. Both the Calogero model 
and the compactified free-boson CFT are theories parametrised by a single parameter (the coupling constant and the compactification radius $R$ respectively). The scaling dimensions of the charged operators are dependent on that single parameter, e.g. the operator $\Phi$ (arising from the $\Psi \Psi$ OPE) in the Calogero model and $e^{i \frac{1}{R} X}$ of the compactified free-boson CFT, where $X$ is the fundamental scalar field. That the $\Psi \Psi$ OPE involves only one primary operator $\Phi$ is reminiscent of the fact that the OPE of $e^{i \frac{1}{R} X} e^{i \frac{1}{R} X}$ involves only one primary operator, $e^{i \frac{2}{R} X}$.

This analogy may be more than superficial: both the Calogero model and the compactified free-boson CFT can be embedded into the sine-Gordon model. As is well-known, the IR limit of the sine-Gordon model (for a range of the coupling constant) is described by the compactified free-boson CFT. (See, for example, [86] and references therein.) On the other hand, one can first take the non-relativistic limit of the sine-Gordon model $[87,88]^{27}$ to obtain a model of two kinds of interacting non-relativistic particles (the solitons and the anti-solitons of the original sine-Gordon model). The pair potential between solitons (or anti-solitons) in this limit has the form $\sim 1 / \sinh ^{2}\left(r / r_{0}\right)$, and that between a soliton and an anti-soliton has the form $\sim-1 / \cosh ^{2}\left(r / r_{0}\right)$. By taking a further limit where the length scale of the non-relativistic model vanishes, one finds that the solitons and anti-solitons decouple from each other, and the interactions among each of them are described by the Calogero model. Thus, the Calogero model and the compactified free-boson CFT can be realised as different limits of the sine-Gordon model.

As is well known, it is possible to compute correlation functions of minimal model CFTs, applying a certain projection to the compactified free-boson CFT. (See for example chapter 9 of [90].) In particular, correlation functions of the critical two-dimensional Ising model can be calculated by taking the "square root" of the compactified free-boson CFT with a special coupling [91-99]. It may be possible to obtain correlation functions of various $z=2$ Schrödinger invariant theories starting from the correlation functions of the Calogero model. In particular, correlation functions of the Glauber model [2], a model describing the dynamical critical behaviour of the Ising model, in one space and one time dimension at criticality may be computed starting from the Calogero model. The Glauber model in $d=1+1$ at criticality has the $z=2$ scale invariant behaviour. (See for example section 10.2 of [11].) The model is exactly solvable in the sense that its partition function can be computed [100] via the mapping to free fermions. This is analogous to the mapping of the two-dimensional Ising model to Majorana fermions [101]. For the Ising model, one can calculate the correlation functions by further rewriting the Majorana fermions as the "square root" of massless Dirac fermions, which in turn is equivalent to the compactified free-boson CFT (with a specific coupling constant) via bosonisation. One may be able to compute general correlation functions of the Glauber model in a similar manner using the Calogero model. We note that the two-point functions of the fundamental spin operator of the Glauber model in 1+1-dimension has been computed [2] and verified to have the form dictated by the Schrödinger symmetry at criticality [32]. Some correlation functions

\footnotetext{
${ }^{27}$ More precisely, in order to retain the nontrivial S-matrix, the non-relativistic limit should be defined as a scaling limit in which the energies of the particles and a parameter of the sine-Gordon theory are going to zero, while the ratios between them are fixed. The precise form of the scaling limit can be found in [89].
} 
related to the three-point functions were computed and it was found that there exists an operator with dimension $\Delta=3$ (in addition to the fundamental spin operator with dimension $\Delta=\frac{1}{2}$ ) $[84,102]$. It is tempting to conjecture that the Calogero model with $\nu=\frac{3}{2}$, in which case the dimension of $\Phi$ becomes $\Delta=\frac{3}{2}+\nu=3$, is relevant for the Glauber model, just like the compactified free-boson CFT with a specific compactification radius is relevant for the two-dimensional Ising model.

Note that $\nu=\frac{3}{2}$ is one of the special "degenerate" cases of the Calogero model $(\nu=$ $\left.-\frac{1}{2}, \frac{1}{2}, \frac{3}{2}, \ldots\right)$ in which the asymptotic series associated with the "t-channel" decomposition truncates. The relation of the Calogero model to the sine-Gordon model (and the system of particles interacting with a $1 / \cosh ^{2} r$ pair potential) may shed light on these special points and the spectrum of zero-charge operators. As is well-known, in the sine-Gordon model, a soliton and an anti-soliton can form a bound state. The number of bound states takes the value $n=0,1,2, \ldots$, depending on the parameter of the sine-Gordon theory. At the special values of the parameter where the number of bound states changes discontinuously, the reflection coefficients between a soliton and an anti-soliton vanish. These special values of the parameters are reminiscent of the special cases, $\nu=-\frac{1}{2}, \frac{1}{2}, \frac{3}{2}, \ldots$, of the Calogero model where the asymptotic series truncates. We speculate that at these special points the "multiplicity" of the zero-charge operators also change discontinuously.

Generalisations. We computed the four-point function by reducing it to the two-particle problem. The integrability of the Calogero model means that one has a certain analytic control over the three- (or more) particle sector. Exploiting the integrability, therefore, it should be possible to calculate six-point functions of the fundamental fields (more precisely the correlation functions with three $\Psi$ 's and three $\bar{\Psi}$ 's), and to study the $\Psi \Phi$ OPE.

One can introduce a three-body interaction to the Calogero model without destroying the integrability in the three-particle problem [103, 104]. Studying such a deformation would be interesting. The deformation will not affect the physics of the two-particle sector, and hence the results of our paper. However, the six-point functions of the fundamental fields and hence the $\Psi \Phi$ OPE will be deformed.

Another interesting variant of the Calogero model is the so-called $\mathrm{B}_{N^{-}}$-type Calogero model. See, for reviews, $[105,106]$. The model can be considered as the Calogero model put on a semi-infinite line with an appropriate boundary condition, which preserves the integrability of the model. We expect that the $\mathrm{B}_{N}$-type Calogero model (around the true vacuum) will exhibit a $z=2$ anisotropic surface critical behaviour and provide a nontrivial yet tractable example of the $z=2$ analogue of a CFT with a boundary.

The integrability of the Calogero model allows one to compute the correlation functions around the finite-density vacuum. (See, for example, [71] and references therein.) The finite-density vacuum breaks the $z=2$ scale invariance spontaneously. It would be interesting to study the finite-density correlation functions from the point of view of the broken $z=2$ scale invariance and Schrödinger invariance. (For a review of spontaneous breaking of the Schrödinger symmetry, see [107].) The IR limit of the Calogero model at finite-density is described by a $c=1$ CFT [108-113]. Thus the finite-density correlation functions of the Calogero model should interpolate between the $z=2$ scale invariant cor- 
relation functions studied in this paper in the UV limit and the $z=1, c=1 \mathrm{CFT}$ in the IR limit.

The Calogero model is inherently related to a system of anyons, which is a $z=2$ Schrödinger invariant model in one time and two space dimensions. (See, for example, [44] and references therein). In particular, the Calogero model is equivalent to a system of anyons restricted to the lowest Landau levels [110, 114-121]. It would be interesting to study the implications of our exact four-point function for the system of anyons.

In this paper we focused on the case with one space dimension. However, the Schrödinger symmetry exists for any number of space dimensions when non-relativistic particles are interacting with a pair potential of the form $1 / r^{2}$ [62]. We do not expect these models in general to be integrable in the conventional sense. However, since our analysis of the four-point function is associated only with the two-particle sector of the model, the computation of the four-point function in higher space dimensions appears feasible. It would be interesting to consider the properties of the OPE, including the OPE associativity, for this higher dimensional system with the Schrödinger symmetry.

Finally, finding an anisotropic scale invariant quantum field theory model with $z \neq 2$ but with exactly computable OPEs is an interesting open problem.

We hope that our analysis provides a starting point of better understanding of fixed points of the renormalisation group for anisotropic theories, and of uncovering a rich structure of solvable models with $z=2$ scale invariance.

\section{Acknowledgments}

We would like to thank Sinya Aoki, Yasuyuki Hatsuda, Shinobu Hikami, Yasuaki Hikida, Masazumi Honda, Masaru Hongo, Stefano Kovacs, Yoichi Kazama, Shota Komatsu, Wenliang Li, Jonathan Miller, Takeshi Morita, Keita Nii, Yoshitaka Okuyama, Norisuke Sakai, Yuta Sekino, Shigeki Sugimoto, Kenta Suzuki, Kotaro Tamaoka, Tadashi Takayanagi, Yuya Tanizaki, Seiji Terashima, Tomonori Ugajin for encouragement, discussions and useful comments.

This work was supported by JSPS KAKENHI (Grant Nos. KAKEN-19H01896, -20K03955, and -20K03796).

\section{A Schrödinger symmetry}

We list here all nonzero commutators in the algebra associated with the Schrödinger symmetry. The members of the algebra are the time translation, the space translations, the angular momenta, a U(1) charge, the dilation, and the spacelike and timelike "special conformal transformations": $H, P_{i}, D, M_{i j}=-M_{j i}, N, K_{i}, C$. Here we used the label $i=1, \cdots, d$ where $d$ is the number of space dimensions. For $d=1$, generators $M_{i j}$ do not exist. The scaling dimensions of these generators are reflected in,

$$
\begin{aligned}
{[D, H] } & =2 i H, \\
{\left[D, P_{i}\right] } & =i P_{i},
\end{aligned}
$$




$$
\begin{aligned}
{\left[D, K_{i}\right] } & =-i K_{i}, \\
{[D, C] } & =-2 i C .
\end{aligned}
$$

The nonzero commutators involving $M_{i j}$ show the transformation properties of the generators under the spatial rotation,

$$
\begin{aligned}
{\left[M_{i j}, P_{k}\right] } & =i\left(\delta_{i k} P_{j}-\delta_{j k} P_{i}\right) \\
{\left[M_{i j}, K_{k}\right] } & =i\left(\delta_{i k} K_{j}-\delta_{j k} K_{i}\right) \\
{\left[M_{i j}, M_{k l}\right] } & =i\left(\delta_{i k} M_{j l}-\delta_{j k} M_{i l}-\delta_{i l} M_{j k}+\delta_{j l} M_{i k}\right) .
\end{aligned}
$$

The remaining non-vanishing commutation relations are

$$
\begin{aligned}
{[H, C] } & =-i D, \\
{\left[C, P_{i}\right] } & =i K_{i}, \\
{\left[H, K_{i}\right] } & =-i P_{i}, \\
{\left[K_{i}, P_{j}\right] } & =i N \delta_{i j} .
\end{aligned}
$$

\section{B Propagator in $1 / r^{2}$ potential}

In this appendix, we compute the propagator for the Hamiltonian, (2.1)

$$
H_{\mathrm{rel}}=-\partial_{r}^{2}+\frac{\lambda(\lambda-1)}{r^{2}}
$$

corresponding to a particle in an external potential $1 / r^{2}$ where $r>0$. The boundary condition for $r \rightarrow 0$ is $\Psi \sim r^{\lambda}$.

The Schrödinger equation for an energy eigenstate $\Psi(r)$ with energy $E=k^{2}(k>0)$ is

$$
-\Psi^{\prime \prime}+\frac{\lambda(\lambda-1)}{r^{2}} \Psi=k^{2} \Psi
$$

For $r \rightarrow+\infty, \Psi(r)$ asymptotes to a linear combination of $e^{ \pm i k r}$.

A simple redefinition

$$
\begin{aligned}
\Psi & =\sqrt{r} w, \\
z & =k r,
\end{aligned}
$$

leads to

$$
z^{2} \frac{d^{2} w}{d z^{2}}+z \frac{d w}{d z}+\left(z^{2}-\left(\lambda-\frac{1}{2}\right)^{2}\right) w=0
$$

which is Bessel's equation [78, (10.2.1)] with $\nu=\lambda-\frac{1}{2}$. Hence the solution to the Schrödinger equation with the desired behaviour at $r \rightarrow 0, \Psi \sim r^{\lambda}$, is

$$
\Psi(r)=N_{k} \sqrt{r} J_{\nu}(k r) .
$$


We shall use the bra-ket notation,

$$
\langle r \mid k\rangle=N_{k} \sqrt{r} J_{\nu}(k r),
$$

where $k>0$.

The normalisation constant $N_{k}$ is fixed by the requirement that $|k\rangle$ 's should give a complete orthonormal basis (with the correct boundary condition)

$$
\left\langle k^{\prime} \mid k\right\rangle=\delta\left(k^{\prime}-k\right) .
$$

Using an integral formula [78, (10.22.67)]

$$
\int_{0}^{\infty} t \exp \left(-p^{2} t^{2}\right) J_{\nu}(a t) J_{\nu}(b t) \mathrm{d} t=\frac{1}{2 p^{2}} \exp \left(-\frac{a^{2}+b^{2}}{4 p^{2}}\right) I_{\nu}\left(\frac{a b}{2 p^{2}}\right),
$$

we see that

$$
\left\langle k^{\prime} \mid k\right\rangle=\overline{N_{k^{\prime}}} N_{k} \int_{0}^{\infty} \sqrt{r} J_{\nu}\left(k^{\prime} r\right) \sqrt{r} J_{\nu}(k r) d r
$$

vanishes for $k \neq k^{\prime}$ and is IR divergent for $k=k^{\prime}$. A natural IR cut-off can be introduced:

$$
\overline{N_{k^{\prime}}} N_{k} \int_{0}^{\infty} \sqrt{r} J_{\nu}\left(k^{\prime} r\right) \sqrt{r} J_{\nu}(k r) e^{-\alpha r^{2}} d r
$$

where we are interested in the limit $\alpha \rightarrow 0$ in the end. This equals

$$
\begin{aligned}
\overline{N_{k^{\prime}}} N_{k} \times \frac{1}{2 \alpha} e^{-\frac{k^{2}+k^{\prime 2}}{4 \alpha}} \times I_{\nu}\left(\frac{k k^{\prime}}{2 \alpha}\right) & \approx \overline{N_{k^{\prime}}} N_{k} \times \frac{1}{2 \alpha} e^{-\frac{k^{2}+k^{\prime 2}}{4 \alpha}} \times e^{\frac{k k^{\prime}}{2 \alpha}} \frac{1}{\sqrt{2 \pi \frac{k k^{\prime}}{2 \alpha}}} \\
& =\overline{N_{k^{\prime}}} N_{k} \times \frac{1}{\sqrt{2 \pi k k^{\prime}}} \frac{1}{\sqrt{2 \alpha}} e^{-\frac{\left(k-k^{\prime}\right)^{2}}{4 \alpha}} \\
& \approx\left|N_{k}\right|^{2} \times \frac{1}{k} \delta\left(k-k^{\prime}\right),
\end{aligned}
$$

using (B.9) and (4.15). Thus we obtain

$$
N_{k}=\sqrt{k}
$$

Finally, the propagator in $1 / r^{2}$ potential is,

$$
\begin{aligned}
\left\langle r^{\prime}\left|e^{-H_{\mathrm{rel}} t}\right| r\right\rangle & =\int_{0}^{+\infty}\left\langle r^{\prime} \mid k\right\rangle e^{-k^{2} t}\langle k \mid r\rangle d k \\
& =\int_{0}^{+\infty} k e^{-k^{2} t \sqrt{r^{\prime}}} J_{\nu}\left(k r^{\prime}\right) \sqrt{r} J_{\nu}(k r) d k \\
& =\sqrt{r r^{\prime}} \frac{1}{2 t} e^{-\frac{r^{2}+r^{\prime 2}}{4 t}} I_{\nu}\left(\frac{r r^{\prime}}{2 t}\right)
\end{aligned}
$$

using (B.9).

If $t$ is very small, the propagator reduces to

$$
\sqrt{\frac{1}{4 \pi t}} e^{-\frac{\left(r^{\prime}-r\right)^{2}}{4 t}}
$$

which coincides with the free particle propagator with the reduced mass $m=\frac{1}{2}$, as it should be. 


\section{Details of the computation of the three-point function $\langle\Psi \Psi \bar{\Phi}\rangle$}

In this appendix, we supply the details of the computation of the three-point function $\langle\Psi \Psi \bar{\Phi}\rangle$ in general position. We also give the comparison to the generic form of the threepoint function derived by Henkel $[32,33]$ and elaborate on the properties of the Schrödinger invariant quantity which we denote $w$.

\section{C.1 Evaluation of the integral representation}

We begin with the integral representation of the three-point function (4.73),

$$
\begin{aligned}
& \left\langle\Psi\left(t+t^{\prime}, x_{4}\right) \Psi\left(t, x_{3}\right) \bar{\Phi}(0,0)\right\rangle \\
& \quad=\frac{1}{2^{\nu+1} \pi^{\frac{3}{4}} \sqrt{\Gamma(\nu+1)}} \frac{1}{\sqrt{t^{\prime}}} \frac{1}{t^{\frac{3}{2}+\nu}} \int e^{-\frac{x_{4^{\prime} 3}^{2}+\left(x_{4^{\prime}}+x_{3}\right)^{2}}{4 t}} \times\left|x_{4^{\prime} 3}\right|^{\nu+\frac{1}{2}} \times e^{-\frac{1}{2} \frac{x_{44^{\prime}}^{2}}{t^{\prime}}} d x_{4^{\prime}} .
\end{aligned}
$$

We evaluate this integral as follows. First, we extract the dependence of the integrand on $x=x_{34^{\prime}}$,

$$
\begin{aligned}
& \left\langle\Psi\left(t+t^{\prime}, x_{4}\right) \Psi\left(t, x_{3}\right) \bar{\Phi}(0,0)\right\rangle \\
= & \frac{1}{2^{\nu+1} \pi^{\frac{3}{4}} \sqrt{\Gamma(\nu+1)}} \frac{1}{\sqrt{t^{\prime}}} \frac{1}{t^{\frac{3}{2}+\nu}} e^{-\frac{x_{3}^{2}}{t}} e^{-\frac{x_{43}^{2}}{2 t^{\prime}}} \int_{-\infty}^{\infty}|x|^{\nu+\frac{1}{2}} e^{-\frac{1}{2}\left(\frac{1}{t}+\frac{1}{t^{\prime}}\right) x^{2}} e^{\left(\frac{x_{3}}{t}-\frac{x_{43}}{t^{\prime}}\right) x} d x .
\end{aligned}
$$

Separating the contribution from $x>0$ and $x<0$, we get,

$$
\begin{aligned}
\langle\Psi(t+ & \left.\left.t^{\prime}, x_{4}\right) \Psi\left(t, x_{3}\right) \bar{\Phi}(0,0)\right\rangle \\
= & \frac{1}{2^{\nu+1} \pi^{\frac{3}{4}} \sqrt{\Gamma(\nu+1)}} \sqrt{\frac{t^{\nu+\frac{1}{2}}}{\left(t+t^{\prime}\right)^{\nu+\frac{3}{2}} t^{\nu+\frac{3}{2}}}} e^{-\frac{x_{3}^{2}}{t}-\frac{x_{43}^{2}}{2 t^{\prime}}} \\
& \times\left(\int_{0}^{\infty} e^{-\frac{1}{2} y^{2}+w y} y^{\nu+\frac{1}{2}} d y+\int_{0}^{\infty} e^{-\frac{1}{2} y^{2}-w y} y^{\nu+\frac{1}{2}} d y\right),
\end{aligned}
$$

where

$$
\begin{aligned}
y & =\sqrt{\frac{t+t^{\prime}}{t t^{\prime}}} x, \\
w & =\sqrt{\frac{t t^{\prime}}{t+t^{\prime}}}\left(\frac{x_{3}}{t}-\frac{x_{43}}{t^{\prime}}\right) .
\end{aligned}
$$

\section{C.2 Relabelling and properties of $w$}

We will write the integrals in the last line of (C.2) using the parabolic cylinder functions [78, section 12], which in turn can be expressed using the confluent hypergeometric functions. Before doing so, we will relabel the $x, t$ coordinates and check whether the result obtained is consistent with the general form $[32,33]$ of three-point functions dictated by the Schrödinger symmetry. To do this we slightly modify our notation to bring the three-point 
function $\left\langle\Psi\left(t+t^{\prime}, x_{4}\right) \Psi\left(t, x_{3}\right) \bar{\Phi}(0,0)\right\rangle$ into the form $\left\langle\Psi\left(t_{3}, x_{3}\right) \Psi\left(t_{2}, x_{2}\right) \bar{\Phi}\left(t_{1}, x_{1}\right)\right\rangle$. Thus, we relabel as, $x_{4} \rightarrow x_{31}, x_{3} \rightarrow x_{21}, t^{\prime} \rightarrow t_{32}, t \rightarrow t_{21}$. The result is,

$$
\begin{aligned}
\left\langle\Psi\left(t_{3}, x_{3}\right) \Psi\left(t_{2}, x_{2}\right) \bar{\Phi}\left(t_{1}, x_{1}\right)\right\rangle & \\
= & \frac{1}{2^{\nu+1} \pi^{\frac{3}{4}} \sqrt{\Gamma(\nu+1)}} \sqrt{\frac{t_{32}^{\nu+\frac{1}{2}}}{t_{31}^{\nu+\frac{3}{2}} t_{21}^{\nu+\frac{3}{2}}}} e^{-\frac{x_{21}^{2}}{t_{21}}-\frac{x_{32}^{2}}{2 t_{32}}} \\
& \times\left(\int_{0}^{\infty} e^{-\frac{1}{2} y^{2}+w y} y^{\nu+\frac{1}{2}} d y+\int_{0}^{\infty} e^{-\frac{1}{2} y^{2}-w y} y^{\nu+\frac{1}{2}} d y\right),
\end{aligned}
$$

with

$$
w=\left(\frac{x_{21}}{t_{21}}-\frac{x_{32}}{t_{32}}\right) \sqrt{\frac{t_{21} t_{32}}{t_{31}}} .
$$

The integral converges since $\nu \geq-\frac{1}{2},(2.2)$. We recall that we chose $t_{3}>t_{2}>t_{1}$ and hence $w \in \mathbb{R}$. It is worthwhile to discuss some properties of the "cross-ratio" $w$ which is invariant under the Schrödinger symmetry. For $t_{3}>t_{2}>t_{1}$ we have $w^{2} \geq 0$, and $w^{2}=0$ holds if and only if the spacetime points $1,2,3$ are aligned on a straight line. It is easy to show the identity

$$
x_{12} t_{23}-x_{23} t_{12}=x_{23} t_{31}-x_{31} t_{23}=x_{31} t_{12}-x_{12} t_{31},
$$

by direct computation. It is amusing to note that the quantity in (C.7) is twice the "area" of a triangle spanned by the spacetime points $1,2,3$ up to sign. It is completely antisymmetric in the labels $1,2,3$. It follows that

$$
\begin{aligned}
\frac{\left(x_{12} t_{23}-x_{23} t_{12}\right)^{2}}{t_{12} t_{23} t_{31}} & =\frac{\left(x_{23} t_{31}-x_{31} t_{23}\right)^{2}}{t_{12} t_{23} t_{31}}=\frac{\left(x_{31} t_{12}-x_{12} t_{31}\right)^{2}}{t_{12} t_{23} t_{31}} \\
& =-\frac{x_{12}^{2}}{t_{12}}-\frac{x_{23}^{2}}{t_{23}}-\frac{x_{31}^{2}}{t_{31}} \\
& =\frac{x_{32}^{2}}{t_{32}}-\frac{x_{31}^{2}}{t_{31}}+\frac{x_{21}^{2}}{t_{21}} \\
& =w^{2}
\end{aligned}
$$

We note that $w^{2}$ again is completely anti-symmetric in the labels $1,2,3$. In section 3.3 , we used the notation $v=\frac{1}{2} w^{2}$.

\section{C.3 Comparison to the general form of three-point functions dictated by Schrödinger symmetry}

The standard form of the three-point function in a Schrödinger invariant theory is, ${ }^{28}$

$$
\begin{aligned}
& \left\langle\mathcal{O}_{3}\left(t_{3}, x_{3}\right) \mathcal{O}_{2}\left(t_{2}, x_{2}\right) \mathcal{O}_{1}\left(t_{1}, x_{1}\right)\right\rangle \\
= & t_{31}^{-\frac{\Delta_{3}+\Delta_{1}-\Delta_{2}}{2}} t_{21}^{-\frac{\Delta_{2}+\Delta_{1}-\Delta_{3}}{2}} t_{32}^{-\frac{\Delta_{3}+\Delta_{2}-\Delta_{1}}{2}} e^{-\frac{\left|N_{2}\right|}{2} \frac{x_{21}^{2}}{t_{21}}-\frac{\left|N_{3}\right|}{2} \frac{x_{31}^{2}}{t_{31}}} F_{123}\left(w^{2}\right),
\end{aligned}
$$

\footnotetext{
${ }^{28}$ Our convention differs slightly from that of [33] in that we adopt the Euclidean statistical field theory convention rather than the Minkowski one. We assume $t_{3}>t_{2}>t_{1}$.
} 
where $F_{123}$ is an arbitrary scaling function which generically is not fixed by the Schrödinger symmetry alone. The quantum numbers $N_{i}(i=1,2,3)$ are the charges of the operators $\mathcal{O}_{i}$ associated with a $\mathrm{U}(1)$-symmetry present for theory with the Schrödinger symmetry. They satisfy $N_{1}+N_{2}+N_{3}=0$, and $N_{1}>0, N_{2}<0, N_{3}<0$. For the three-point function studied here, we have $\mathcal{O}_{3}=\Psi, \mathcal{O}_{2}=\Psi, \mathcal{O}_{1}=\bar{\Phi}$ and $\Delta_{3}=\frac{1}{2}, \Delta_{2}=\frac{1}{2}, \Delta_{1}=\frac{3}{2}+\nu$, $N_{3}=-1, N_{2}=-1, N_{1}=2$.

To compare with the standard form, it is convenient to rewrite (C.5) using (C.8),

$$
\begin{aligned}
\left\langle\Psi\left(t_{3}, x_{3}\right) \Psi\left(t_{2}, x_{2}\right) \bar{\Phi}\left(t_{1}, x_{1}\right)\right\rangle & \\
= & \frac{1}{2^{\nu+1} \pi^{\frac{3}{4}} \sqrt{\Gamma(\nu+1)}} \sqrt{\frac{t_{32}^{\nu+\frac{1}{2}}}{t_{31}^{\nu+\frac{3}{2}} t_{21}^{\nu+\frac{3}{2}}} e^{-\frac{x_{21}^{2}}{2 t_{21}}-\frac{x_{31}^{2}}{2 t_{31}}}} \\
& \times e^{-\frac{1}{2} w^{2}}\left(\int_{0}^{\infty} e^{-\frac{1}{2} y^{2}+w y} y^{\nu+\frac{1}{2}} d y+\int_{0}^{\infty} e^{-\frac{1}{2} y^{2}-w y} y^{\nu+\frac{1}{2}} d y\right) .
\end{aligned}
$$

The last line combined with the numerical prefactor is the scaling function $F_{123}$.

\section{C.4 Three-point function in terms of parabolic cylinder functions and conflu- ent hypergeometric functions}

The integral appearing in (C.10) can be written [78, (12.5.1)]

$$
\int_{0}^{\infty} e^{-\frac{y^{2}}{2}+w y} y^{\nu+\frac{1}{2}} d y=\Gamma\left(\nu+\frac{3}{2}\right) e^{\frac{1}{4} w^{2}} U(\nu+1,-w),
$$

using the parabolic cylinder function $U(a, z)$. Thus we obtain

$$
\begin{aligned}
\left\langle\Psi\left(t_{3}, x_{3}\right) \Psi\left(t_{2}, x_{2}\right) \bar{\Phi}\left(t_{1}, x_{1}\right)\right\rangle & \\
= & \frac{\Gamma\left(\nu+\frac{3}{2}\right)}{2^{\nu+1} \pi^{\frac{3}{4}} \sqrt{\Gamma(\nu+1)}} \sqrt{\frac{t_{32}{ }^{\nu+\frac{1}{2}}}{t_{31}^{\nu+\frac{3}{2}} t_{21}^{\nu+\frac{3}{2}}}} e^{-\frac{x_{21}^{2}}{2 t_{21}}-\frac{x_{31}^{2}}{2 t_{31}}} \\
& \times e^{-\frac{1}{4} w^{2}}(U(\nu+1,-w)+U(\nu+1, w)) .
\end{aligned}
$$

We observe that the last line is even in $w$. We rewrite the above formula in terms of a parabolic hyperbolic function, $u_{1}$ in the notation of [78], which is even in $w$. We will then rewrite the formula in terms of the confluent hypergeometric functions. This will be useful to check against the result by Golkar and Son [52], and also to study simplifying limits, namely the free boson limit $\left(\nu=-\frac{1}{2}\right.$, appendix E.2), and the limit $t_{32} \rightarrow 0$, which we already computed in section 4.3.1.

From (12.4.1) and (12.2.6) of [78],

$$
\begin{aligned}
& U(a, z)=U(a, 0) u_{1}(a, z)+U^{\prime}(a, 0) u_{2}(a, z), \\
& U(a, 0)=\frac{\sqrt{\pi}}{2^{\frac{1}{2} a+\frac{1}{4}} \Gamma\left(\frac{3}{4}+\frac{1}{2} a\right)}
\end{aligned}
$$


where $u_{1}$ and $u_{2}$ are respectively even and odd in $z$, we obtain

$$
U(a, z)+U(a,-z)=\frac{\sqrt{\pi}}{2^{\frac{1}{2} a-\frac{3}{4}} \Gamma\left(\frac{3}{4}+\frac{1}{2} a\right)} u_{1}(a, z),
$$

and hence

$$
\begin{aligned}
& \left\langle\Psi\left(t_{3}, x_{3}\right) \Psi\left(t_{2}, x_{2}\right) \bar{\Phi}\left(t_{1}, x_{1}\right)\right\rangle \\
& =\frac{\Gamma\left(\nu+\frac{3}{2}\right)}{2^{\frac{3}{2} \nu+\frac{3}{4}} \pi^{\frac{1}{4}} \sqrt{\Gamma(\nu+1)} \Gamma\left(\frac{\nu}{2}+\frac{5}{4}\right)} \sqrt{\frac{t_{32}{ }^{\nu+\frac{1}{2}}}{t_{31}^{\nu+\frac{3}{2}} t_{21}^{\nu+\frac{3}{2}}}} e^{-\frac{x_{21}^{2}}{2 t_{21}}} e^{-\frac{x_{31}^{2}}{2 t_{31}}} \\
& \times e^{-\frac{1}{4} w^{2}} u_{1}(\nu+1, w) .
\end{aligned}
$$

One can rewrite the result in terms of the confluent hypergeometric function $M(a, b, x)$ using $[78,(12.7 .12)]$

$$
u_{1}(a, z)=e^{-\frac{1}{4} z^{2}} M\left(\frac{1}{2} a+\frac{1}{4}, \frac{1}{2}, \frac{1}{2} z^{2}\right)=e^{\frac{1}{4} z^{2}} M\left(-\frac{1}{2} a+\frac{1}{4}, \frac{1}{2},-\frac{1}{2} z^{2}\right) .
$$

This leads, finally, to

$$
\begin{aligned}
& \left\langle\Psi\left(t_{3}, x_{3}\right) \Psi\left(t_{2}, x_{2}\right) \Phi\left(t_{1}, x_{1}\right)\right\rangle \\
& =Q \frac{\Gamma\left(\nu+\frac{3}{2}\right)}{2^{\frac{3}{2} \nu+\frac{3}{4}} \pi^{\frac{1}{4}} \sqrt{\Gamma(\nu+1)} \Gamma\left(\frac{\nu}{2}+\frac{5}{4}\right)} \sqrt{\frac{t_{32}{ }^{\nu+\frac{1}{2}}}{t_{31}^{\nu+\frac{3}{2}}} t_{21}^{\nu+\frac{3}{2}}} e^{-\frac{x_{21}^{2}}{2 t_{21}} e^{-\frac{x_{31}^{2}}{2 t_{31}}}} \\
& \quad \times M\left(-\frac{\nu}{2}-\frac{1}{4}, \frac{1}{2},-\frac{1}{2} w^{2}\right)
\end{aligned}
$$

and,

$$
\begin{aligned}
& \left\langle\Psi\left(t_{3}, x_{3}\right) \Psi\left(t_{2}, x_{2}\right) \bar{\Phi}\left(t_{1}, x_{1}\right)\right\rangle \\
& =\frac{\Gamma\left(\nu+\frac{3}{2}\right)}{2^{\frac{3}{2} \nu+\frac{3}{4}} \pi^{\frac{1}{4}} \sqrt{\Gamma(\nu+1)} \Gamma\left(\frac{\nu}{2}+\frac{5}{4}\right)} \sqrt{\frac{t_{32}{ }^{\nu+\frac{1}{2}}}{t_{31}^{\nu+\frac{3}{2}} t_{21}^{\nu+\frac{3}{2}}} e^{-\frac{x_{21}^{2}}{2 t_{21}}} e^{-\frac{x_{31}^{2}}{2 t_{31}}}} \\
& \quad \times e^{-\frac{w^{2}}{2}} M\left(\frac{\nu}{2}+\frac{3}{4}, \frac{1}{2}, \frac{1}{2} w^{2}\right) .
\end{aligned}
$$

The latter is our final expression for the three-point function, quoted in the main text as (4.74).

\section{C.5 $\quad t_{32} \rightarrow 0$ limit}

As a consistency check, we consider the limit $t_{32} \rightarrow 0$ to compare with the result of section 4.3.1. In this limit, we have $w^{2} \approx \frac{x_{32}^{2}}{t_{32}} \rightarrow+\infty$. Applying the asymptotic formula of the confluent hypergeometric function $[78,(13.7 .1) \text { and (13.2.4) }]^{29}$

$$
M(a, b, x) \sim \frac{e^{x} x^{a-b}}{\Gamma(a) \Gamma(b)} \sum_{s=0}^{\infty} \frac{(1-a)_{s}(b-a)_{s}}{s !} x^{-s}, \quad(x \rightarrow+\infty, x \in \mathbb{R}),
$$

\footnotetext{
${ }^{29}$ The asymptotic formula is invalid when $a$ and $b$ are non-positive integers. These exceptional cases are automatically avoided in our use of the formula. See (4.74) or equivalently (C.19).
} 
to the three-point function (4.74) (equivalently (C.19)), we obtain

$$
\begin{aligned}
& \left\langle\Psi\left(t, x_{3}\right) \Psi\left(t, x_{2}\right) \bar{\Phi}\left(0, x_{1}\right)\right\rangle \\
& =\frac{\Gamma\left(\nu+\frac{3}{2}\right)}{2^{\frac{3}{2} \nu+\frac{1}{4}}(4 \pi)^{\frac{1}{4}} \sqrt{\Gamma(\nu+1)} \Gamma\left(\frac{\nu}{2}+\frac{5}{4}\right)} t^{-\left(\nu+\frac{3}{2}\right)} e^{-\frac{x_{21}^{2}}{2 t}} e^{-\frac{x_{31}^{2}}{2 t}} \\
& \quad \times\left(\frac{1}{2} x_{32}^{2}\right)^{\frac{\nu}{2}+\frac{1}{4}} \frac{\Gamma\left(\frac{1}{2}\right)}{\Gamma\left(\frac{\nu}{2}+\frac{3}{4}\right)},
\end{aligned}
$$

where we have written $t=t_{31}=t_{21}$, and put $t_{1}=0$. This indeed agrees with (4.69) which we obtained in section 4.3.1 directly from the pairwise equal-time four-point function (3.13) since we have

$$
\frac{\sqrt{\pi} \Gamma\left(\nu+\frac{3}{2}\right)}{2^{\nu+\frac{1}{2}} \Gamma\left(\frac{\nu}{2}+\frac{5}{4}\right) \Gamma\left(\frac{\nu}{2}+\frac{3}{4}\right)}=1,
$$

which follows from the duplication formula [78, (5.5.5)]

$$
\Gamma(2 z)=\pi^{-1 / 2} 2^{2 z-1} \Gamma(z) \Gamma\left(z+\frac{1}{2}\right) .
$$

\section{Golkar and Son's analysis in Euclidean signature}

Golkar and Son showed [52] that the form of the scaling function appearing in the threepoint function in a Schrödinger invariant theory is severely restricted when the scaling dimension of one of the operators equals the special value, $\Delta=\frac{d}{2}$, where $d$ is the number of spacelike dimensions. The scaling function satisfies (except for a simple prefactor) the confluent hypergeometric equation. Their analysis was done in Minkowski signature. Since how the analysis takes over to Euclidean signature is not entirely trivial, in this appendix we give the Euclidean version of the analysis of Golkar and Son. ${ }^{30}$ In this appendix $d$ is arbitrary and we write $x=(t, \boldsymbol{x})$.

The solution to the differential equation contains two arbitrary parameters. In [52] it was advocated that one of the parameters vanishes due to the regularity conditions of the OPE, acting as the boundary conditions of the differential equation. We will also give below a careful discussion of the regularity conditions, in particular, for the case $d=1$. We will see that for that case, the regularity conditions are weaker and do not imply the vanishing of the parameter.

\footnotetext{
${ }^{30}$ We note that the notation used in [52] is slightly unusual. They call what is usually called (up to constant multiplication) the confluent hypergeometric function $M(a, b, x)$ (in the notation of [78]) as "a generalised Laguerre polynomial" $L_{n}{ }^{\alpha}(x)$, with $n=-a, b=\alpha+1$. The function is not a polynomial unless $n=-a$ is a non-negative integer. As shown in [52] the parameter $a$ is related to the scaling dimensions of the operators (see (D.22)), and is not an integer, in general.
} 


\section{D.1 Preliminaries}

The operators in the Heisenberg picture are

$$
\mathcal{O}(t, \boldsymbol{x})=U^{-1} \mathcal{O}(0, \mathbf{0}) U
$$

where

$$
U=e^{-H t+i \boldsymbol{P} \cdot \boldsymbol{x}}
$$

It is straightforward to verify

$$
\begin{aligned}
U K_{i} U^{-1} & =K_{i}+i P_{i} t+N x_{i}, \\
U C U^{-1} & =C+i D t+K_{i} x_{i}-H t^{2}+i P_{i} x^{i} t+\frac{1}{2} N \boldsymbol{x}^{2},
\end{aligned}
$$

using the elementary identity

$$
e^{A} B e^{-A}=B+[A, B]+\frac{1}{2 !}[A,[A, B]]+\frac{1}{3 !}[A,[A,[A, B]]]+\cdots,
$$

and the commutation relations given in appendix $\mathrm{A}$.

A primary operator $\mathcal{O}$ in a Schrödinger invariant theory is defined by the conditions

$$
\begin{aligned}
{\left[K_{i}, \mathcal{O}(0)\right] } & =0 \\
{[C, \mathcal{O}(0)] } & =0
\end{aligned}
$$

It follows that

$$
\begin{aligned}
{\left[K_{i}, \mathcal{O}(t, \boldsymbol{x})\right] } & =-t \partial_{i} \mathcal{O}(x)+N_{\mathcal{O}} x_{i} \mathcal{O}(x) \\
{[C, \mathcal{O}(t, \boldsymbol{x})] } & =-\Delta_{\mathcal{O}} t \mathcal{O}-t^{2} \partial_{t} \mathcal{O}-t x^{i} \partial_{i} \mathcal{O}+\frac{1}{2} N_{\mathcal{O}} \boldsymbol{x}^{2} \mathcal{O}
\end{aligned}
$$

where we used

$$
\begin{aligned}
\partial_{i} \mathcal{O}(t, \boldsymbol{x}) & =\left[\mathcal{O}(t, \boldsymbol{x}), i P_{i}\right], \\
\partial_{t} \mathcal{O} & =[H, \mathcal{O}], \\
{[N, \mathcal{O}] } & =N_{\mathcal{O}} \mathcal{O}, \\
{[D, \mathcal{O}(0)] } & =i \Delta_{\mathcal{O}} \mathcal{O}(0) .
\end{aligned}
$$

Here $N_{\mathcal{O}}$ and $\Delta_{\mathcal{O}}$ are the $\mathrm{U}(1)$ charge and the scaling dimension of the operator $\mathcal{O}$.

\section{D.2 OPE coefficients}

We consider general constraints on the OPE coefficients imposed by the Schrödinger symmetry. We consider the OPE $\mathcal{O}_{2} \mathcal{O}_{1}$ and focus on the part proportional to $\mathcal{O}_{3}$, where $\mathcal{O}_{i}$ are scalar primary operators with nonzero U(1) charges. We consider the special case, $\Delta_{3}=\frac{d}{2}$.

We write down explicitly the first few descendants of $\mathcal{O}_{3}$,

$$
\mathcal{O}_{2}(x) \mathcal{O}_{1}(0)=\left.\left(\left(C_{0}(x)+C_{1}^{i}(x) \partial_{i}+C_{2}(x) \partial_{t}+C_{3}^{i j}(x) \partial_{i} \partial_{j}+\cdots\right) \mathcal{O}_{3}\right)\right|_{x=0}+\cdots,
$$

where $t>0$ is assumed. 
By taking the commutators of $K_{i}, C$ with the l.h.s. and r.h.s., we obtain

$$
\begin{aligned}
-t \partial_{i} C_{0}+N_{2} x_{i} C_{0} & =N_{3} C_{1}^{i}, \\
-t \partial_{i} C_{1}^{j}+N_{2} x_{i} C_{1}^{j} & =-C_{2} \delta_{i j}+2 N_{3} C_{3}^{i j}, \\
-\Delta_{2} t C_{0}-t^{2} \partial_{t} C_{0}-t x^{i} \partial_{i} C_{0}+\frac{N_{2}}{2} x^{2} C_{0} & =-\Delta_{3} C_{2}+C_{3}^{j j} N_{3},
\end{aligned}
$$

where we write $N_{i} \equiv N_{\mathcal{O}_{i}},(i=1,2,3)$. Generically, these equations express differential operators acting on $C_{0}$ to give $C_{1}, C_{2}, \cdots$. For the special case $\Delta_{3}=\frac{d}{2},(\mathrm{D} .15)-(\mathrm{D} .17)$ imply a differential equation on the coefficient $C_{0}$ :

$$
t^{2} \partial_{i}^{2} C_{0}+2 N_{3} t^{2} \partial_{t} C_{0}+2 N_{1} t x^{i} \partial_{i} C_{0}+\left(2 N_{3} \Delta_{2}-N_{2} d\right) t C_{0}-N_{2} N_{1} \boldsymbol{x}^{2} C_{0}=0 .
$$

The scale and $\mathrm{SO}(d)$ invariance require $C_{0}$ to have the form

$$
C_{0}(t, \boldsymbol{x})=t^{-\frac{\Delta_{2}+\Delta_{1}-\Delta_{3}}{2}} f\left(\frac{\boldsymbol{x}^{2}}{t}\right) .
$$

Substituting this to (D.18), we obtain, using $N_{3}=N_{2}+N_{1}$,

$$
\begin{aligned}
0= & 4 y \frac{d^{2} f}{d y^{2}}+2 d \frac{d f}{d y}+2\left(N_{1}-N_{2}\right) y \frac{d f}{d y} \\
& +\left(N_{3}\left(\Delta_{2}-\Delta_{1}\right)+\left(N_{1}-N_{2}\right) \frac{d}{2}\right) f-N_{2} N_{1} y f,
\end{aligned}
$$

where $y=\frac{x^{2}}{t}$. This differential equation becomes the confluent hypergeometric equation

$$
z \frac{d^{2} v}{d z^{2}}+(b-z) \frac{d v}{d z}-a v=0,
$$

with

$$
\begin{aligned}
a & =\frac{1}{2}\left(\Delta_{1}-\Delta_{2}+\frac{d}{2}\right), \\
b & =\frac{d}{2}>0,
\end{aligned}
$$

by a simple transformation $f=e^{-\frac{N_{1}}{2} y} v, z=\frac{N_{3}}{2} y$. Thus we have

$$
C_{0}(t, \boldsymbol{x})=t^{-\frac{\Delta_{2}+\Delta_{1}-\Delta_{3}}{2}} e^{-\frac{N_{1}}{2} \frac{\boldsymbol{x}^{2}}{t}} v\left(\frac{N_{3}}{2} \frac{\boldsymbol{x}^{2}}{t}\right) \text {. }
$$

We assume, for simplicity, that $a$ is not a negative integer. (This can always be met for example by replacing $\left(\mathcal{O}_{1}, \mathcal{O}_{2}\right)$ with $\left(\overline{\mathcal{O}}_{2}, \overline{\mathcal{O}}_{1}\right)$ so that $\Delta_{1}>\Delta_{2}$. $)$ The standard confluent hypergeometric functions $M(a, b, z)$ and $U(a, b, z)$ (in the notation of [78]) are then linearly independent. Hence any solution can be written

$$
v=A M(a, b, z)+B U(a, b, z),
$$

where $A, B$ are constants.

In [52], it was advocated that appropriate regularity conditions on the OPE coefficient imply

$$
B=0 .
$$

This point will be examined in the next subsection D.3. 


\section{D.3 Boundary condition}

Here we will study the regularity conditions of the OPE, leading to boundary conditions on the function $v$ appearing in (D.24). We focus in particular on the possible restrictions on the coefficients $A$ and $B$ in (D.25).

Firstly, we observe that the regularity of $C_{0}$, in the limit $t \rightarrow 0, x \rightarrow 0$ with $\frac{x^{2}}{t}$ fixed at a nonzero finite value, does not impose any conditions on $v$. Hence, if we wish to restrict the form of $v$ we have to consider the limit $\frac{x^{2}}{t} \rightarrow \infty$ (the equal-time OPE) or $\frac{x^{2}}{t} \rightarrow 0$ (the "equal-space" OPE, i.e. the $\left.\mathrm{OPE} \mathcal{O}_{2}(t, \mathbf{0}) \mathcal{O}_{1}(0, \mathbf{0})\right)$.

Let us first examine the latter limit, i.e. the behaviour at fixed $t>0$ and $\boldsymbol{x} \rightarrow \mathbf{0}$. Then $z \rightarrow+0$ and the prefactor in (D.24) behaves as, $e^{-\frac{N_{1}}{2} \frac{x^{2}}{t}} \rightarrow 1$. In this limit, and for the values of $a, b$ relevant for us (D.22) (D.23), we have

$$
\begin{aligned}
M(a, b, z) & =1+O(z), \\
U(a, b, z) & \approx \frac{\Gamma(b-1)}{\Gamma(a)} z^{1-b}, \quad\left(b=\frac{d}{2}, d=1,3,4,5, \cdots\right), \\
U(a, b, z) & \approx-\frac{1}{\Gamma(a)}(\log z+\text { const. }), \quad\left(b=\frac{d}{2}, d=2\right) .
\end{aligned}
$$

We have to distinguish the case $d=1$ and $d=2,3, \cdots$.

For $d=2,3, \cdots, U(a, b, z) \rightarrow \infty$ as $z \rightarrow 0$ whereas $M(a, b, z) \rightarrow 1$. Hence if we require the existence of the "equal-space" OPE, ${ }^{31}$ we obtain

$$
B=0 \text {. }
$$

This is the result of Golkar and Son [52].

For $d=1$, we have $M(a, b, z) \rightarrow 1$ and $U(a, b, z) \rightarrow 0$ as $z \rightarrow 0$. Hence even if we require the existence of the OPE in the limit $\frac{x^{2}}{t} \rightarrow 0$, the coefficients $A$ and $B$ are not constrained. (If we require further that the OPE be non-vanishing then we get $A \neq 0$.)

Let us next examine the behaviour at $z \rightarrow+\infty$, which corresponds to $t \rightarrow 0+$ with fixed $\boldsymbol{x}$, i.e. to the limit of the equal-time OPE. We shall see in fact that the OPE coefficient $C_{0}$ in this limit either diverges or goes to zero. This is not surprising: consider, in the free-field theory, the part of the OPE $\Psi(t, \boldsymbol{x}) \bar{\Psi} \bar{\Psi}(0, \mathbf{0})$ proportional to $\bar{\Psi}(0, \mathbf{0})$. The OPE coefficient is essentially the two-point function $\langle\Psi(t, \boldsymbol{x}) \bar{\Psi}(0, \mathbf{0})\rangle \sim \frac{1}{\sqrt{t}} e^{-\frac{x^{2}}{2 t}}$ and is singular in the limit $t \rightarrow 0+$ with fixed $\boldsymbol{x}$.

The general argument goes as follows. In the limit, $z \rightarrow \infty$, we have

$$
\begin{aligned}
M(a, b, z) & \sim \frac{1}{\Gamma(a)} e^{z} z^{a-b}, \\
U(a, b, z) & \sim z^{-a} .
\end{aligned}
$$

Let us separately consider the $A$ - and $B$ - type solution, i.e. the first and the second term in (D.25), respectively. The $A$-type solution gives, in the limit $t \rightarrow 0+$ with fixed $\boldsymbol{x}$,

$$
C_{0}(t, \boldsymbol{x}) \sim A e^{-\frac{N_{1}}{2} \frac{x^{2}}{t}} e^{\frac{N_{3}}{2} \frac{x^{2}}{t}}=A e^{\frac{N_{2}}{2} \frac{x^{2}}{t}} .
$$

\footnotetext{
${ }^{31}$ We wish to note that it is far from obvious whether the requirement of the existence of the regular "equal-space" OPE is mandatory or not.
} 
Hence if $N_{2}>0, C_{0}$ diverges, and if $N_{2}<0, C_{0}$ goes to zero. (We only write in the above formula the leading exponential behaviour.) For the $B$-type solution, we have

$$
\begin{aligned}
C_{0}(t, \boldsymbol{x}) & \approx B t^{-\frac{\Delta_{2}+\Delta_{1}-\Delta_{3}}{2}} e^{-\frac{N_{1}}{2} \frac{x^{2}}{t}}\left(\frac{\boldsymbol{x}^{2}}{t}\right)^{-\frac{1}{2}\left(\Delta_{1}-\Delta_{2}+\frac{d}{2}\right)} \\
& =B t^{-\Delta_{2}+\frac{d}{2}}\left(\boldsymbol{x}^{2}\right)^{-\frac{1}{2}\left(\Delta_{1}-\Delta_{2}+\frac{d}{2}\right)} e^{-\frac{N_{1}}{2} \frac{x^{2}}{t}},
\end{aligned}
$$

using $\Delta_{3}=\frac{d}{2}$. Again for $N_{1} \neq 0, C_{0}$ either goes to 0 or diverges. Hence, for operators with nonzero charges and $\Delta_{3}=\frac{d}{2}$, the equal-time OPE either diverges or vanishes.

To summarise this subsection, the limit $\frac{x^{2}}{t} \rightarrow+\infty$ (the equal-time OPE) is singular (the OPE coefficient either diverging or vanishing) and does not give constraints on the coefficients $A, B$. If we require the regularity of $\frac{x^{2}}{t} \rightarrow 0$ (the equal-space OPE), we obtain $B=0$ for $d=2,3, \cdots$, but no constraints for $d=1$.

\section{D.4 Three-point function}

We consider the general form of the three-point function of primary operators [32],

$$
\begin{aligned}
& \left\langle\overline{\mathcal{O}}_{3}\left(t_{3}, x_{3}\right) \mathcal{O}_{2}\left(t_{2}, x_{2}\right) \mathcal{O}_{1}\left(t_{1}, x_{1}\right)\right\rangle \\
& \quad=t_{31}^{-\frac{\Delta_{3}+\Delta_{1}-\Delta_{2}}{2}} t_{21}^{-\frac{\Delta_{2}+\Delta_{1}-\Delta_{3}}{2}} t_{32}^{-\frac{\Delta_{3}+\Delta_{2}-\Delta_{1}}{2}} e^{-\frac{\left|N_{2}\right|}{2} \frac{x_{21}^{2}}{t_{21}}-\frac{\left|-N_{3}\right|}{2} \frac{x_{31}^{2}}{t_{31}}} F_{123}\left(w^{2}\right),
\end{aligned}
$$

where $t_{3}>t_{2}>t_{1}$. Here $w$ is the Schrödinger invariant spacetime cross-ratio defined by (C.8). To be specific, we consider the case,

$$
\begin{aligned}
& N_{1}>0, N_{2}<0, N_{3}>0,-N_{3}<0, \\
& N_{1}+N_{2}-N_{3}=0 .
\end{aligned}
$$

(This choice is consistent with the three-point function we studied in section 4.3.2. The comparison is done at the end of this subsection.)

To compare with the OPE coefficient, we set

$$
\begin{aligned}
& \left(t_{1}, \boldsymbol{x}_{1}\right)=(0, \mathbf{0}), \\
& \left(t_{2}, \boldsymbol{x}_{2}\right)=(t, \boldsymbol{x}),
\end{aligned}
$$

and consider the limit $(t, \boldsymbol{x}) \rightarrow(0, \mathbf{0})$ with $\frac{\boldsymbol{x}^{2}}{t}$ fixed. Then we have

$$
w^{2}=\frac{\left(\boldsymbol{x}_{3}-\boldsymbol{x}\right)^{2}}{t_{3}-t}-\frac{\left(\boldsymbol{x}_{3}\right)^{2}}{t_{3}}+\frac{\boldsymbol{x}^{2}}{t} \longrightarrow \frac{\boldsymbol{x}^{2}}{t},
$$

and hence

$$
\begin{aligned}
& \left\langle\overline{\mathcal{O}}_{3}\left(t_{3}, x_{3}\right) \mathcal{O}_{2}\left(t_{2}, x_{2}\right) \mathcal{O}_{1}\left(t_{1}, x_{1}\right)\right\rangle \\
& \quad \longrightarrow t_{3}^{-\Delta_{3}} e^{-\frac{N_{3}}{2} \frac{x_{3}^{2}}{t_{3}}} t^{-\frac{\Delta_{2}+\Delta_{1}-\Delta_{3}}{2}} e^{-\frac{-N_{2}}{2} \frac{x^{2}}{t}} F\left(\frac{x^{2}}{t}\right) .
\end{aligned}
$$

On the other hand, the OPE yields

$$
\begin{aligned}
\left\langle\overline{\mathcal{O}}_{3}(\right. & \left.\left.t_{3}, \boldsymbol{x}_{3}\right) \mathcal{O}_{2}(t, \boldsymbol{x}) \mathcal{O}_{1}(0, \mathbf{0})\right\rangle \\
& \rightarrow\left\langle\overline{\mathcal{O}}_{3}\left(t_{3}, \boldsymbol{x}_{3}\right) \mathcal{O}_{3}(0, \mathbf{0})\right\rangle \times C_{0}(t, \boldsymbol{x}) \\
\quad & t_{3}^{-\Delta_{3}} e^{-\frac{N_{3}}{2} \frac{\boldsymbol{x}_{3}^{2}}{t_{3}}} \times t^{-\frac{\Delta_{2}+\Delta_{1}-\Delta_{3}}{2}} e^{-\frac{N_{1}}{2} \frac{x^{2}}{t}} v\left(\frac{N_{3}}{2} \frac{\boldsymbol{x}^{2}}{t}\right),
\end{aligned}
$$


using (1.4) and (D.24). Note that the contributions from descendants of $\mathcal{O}_{3}$ vanish in this limit.

Comparing (D.41) and (D.42), we finally obtain

$$
\begin{aligned}
F(y) & =e^{-\frac{N_{3}}{2} y} v(y) \\
& =e^{-\frac{N_{3}}{2} y}\left(A M\left(\frac{1}{2}\left(\Delta_{1}-\Delta_{2}+\frac{d}{2}\right), \frac{d}{2}, \frac{N_{3}}{2} y\right)+B U\left(\frac{1}{2}\left(\Delta_{1}-\Delta_{2}+\frac{d}{2}\right), \frac{d}{2}, \frac{N_{3}}{2} y\right)\right),
\end{aligned}
$$

using (D.25), (D.22), (D.23).

Substituting back to (D.35), the three-point function is

$$
\begin{aligned}
& \left\langle\overline{\mathcal{O}}_{3}\left(t_{3}, x_{3}\right) \mathcal{O}_{2}\left(t_{2}, x_{2}\right) \mathcal{O}_{1}\left(t_{1}, x_{1}\right)\right\rangle \\
& =t_{31}^{-\frac{\Delta_{3}+\Delta_{1}-\Delta_{2}}{2}} t_{21}^{-\frac{\Delta_{2}+\Delta_{1}-\Delta_{3}}{2}} t_{32}^{-\frac{\Delta_{3}+\Delta_{2}-\Delta_{1}}{2}} e^{-\frac{\left|N_{2}\right|}{2} \frac{x_{21}^{2}}{t_{21}}-\frac{\left|-N_{3}\right|}{2} \frac{x_{31}^{2}}{t_{31}}} \\
& \quad \times e^{-\frac{N_{3}}{2} w^{2}}\left(A M\left(\frac{1}{2}\left(\Delta_{1}-\Delta_{2}+\frac{d}{2}\right), \frac{d}{2}, \frac{N_{3}}{2} w^{2}\right)+B U\left(\frac{1}{2}\left(\Delta_{1}-\Delta_{2}+\frac{d}{2}\right), \frac{d}{2}, \frac{N_{3}}{2} w^{2}\right)\right) .
\end{aligned}
$$

To compare with our result presented in section 4.3 , we put $\mathcal{O}_{1}=\bar{\Phi}, \mathcal{O}_{2}=\Psi, \overline{\mathcal{O}}_{3}=\Psi$ with $N_{1}=2, N_{2}=-1, N_{3}=1, \Delta_{1}=\frac{3}{2}+\nu, \Delta_{2}=\frac{1}{2}, \Delta_{3}=\frac{1}{2}$, and $d=1$. We see that for our model, the result of our explicit computation (4.74) supports Golkar and Son's ansatz, $B=0$, even if the regularity conditions do not require $B=0$ in $d=1$.

\section{E Free-Boson limit}

In this appendix, we consider the limiting case $\nu=-\frac{1}{2}$, which is the free boson theory.

\section{E.1 Pairwise equal-time four-point function}

Substituting

$$
I_{-\frac{1}{2}}(y)=\sqrt{\frac{2}{\pi}} \frac{1}{\sqrt{y}} \cosh y
$$

into the pairwise equal-time four-point function (3.13) (which is valid for $t>0, x_{21}>$ $0, x_{43}>0$ ), we obtain

$$
\begin{aligned}
& \left\langle\Psi\left(t, x_{4}\right) \Psi\left(t, x_{3}\right) \bar{\Psi}\left(0, x_{2}\right) \bar{\Psi}\left(0, x_{1}\right)\right\rangle \\
& =e^{-\frac{x_{21}^{2}+x_{43}^{2}+\left(x_{3}+x_{4}-x_{1}-x_{2}\right)^{2}}{4 t}} \times \sqrt{\frac{x_{21} x_{43}}{4 \pi t^{3}}} \sqrt{\frac{2}{\pi}} \frac{1}{\sqrt{\frac{x_{21} x_{43}}{2 t}}} \cosh \frac{x_{21} x_{43}}{2 t} \\
& =e^{-\frac{x_{21}^{2}+x_{43}^{2}+\left(x_{3}+x_{4}-x_{1}-x_{2}\right)^{2}}{4 t}} \times \frac{1}{\pi t} \cosh \frac{x_{21} x_{43}}{2 t} \\
& =\frac{1}{2 \pi t}\left(e^{-\frac{\left(x_{21}+x_{43}\right)^{2}+\left(x_{3}+x_{4}-x_{1}-x_{2}\right)^{2}}{4 t}}+e^{-\frac{\left(x_{21}-x_{43}\right)^{2}+\left(x_{3}+x_{4}-x_{1}-x_{2}\right)^{2}}{4 t}}\right) \\
& =\frac{1}{2 \pi t}\left(e^{-\frac{x_{14}^{2}+x_{23}^{2}}{2 t}}+e^{-\frac{x_{13}^{2}+x_{24}^{2}}{2 t}}\right) \\
& =K\left(x_{4}, x_{1} ; t\right) K\left(x_{3}, x_{2} ; t\right)+K\left(x_{4}, x_{2} ; t\right) K\left(x_{3}, x_{1} ; t\right),
\end{aligned}
$$


where $K\left(x^{\prime}, x ; t\right)$ in the last line is the free propagator $(3.20)$. This is the expected result for free bosons.

\section{E.2 Three-point function}

Substituting $\nu=-\frac{1}{2}$ into (4.74), and then using $M(1, b, z)=1$ for general $b$, we obtain

$$
\left\langle\Psi\left(t_{3}, x_{3}\right) \Psi\left(t_{2}, x_{2}\right) \bar{\Phi}\left(t_{1}, x_{1}\right)\right\rangle=\frac{1}{\sqrt{\pi}} \sqrt{\frac{1}{t_{31} t_{21}}} e^{-\frac{x_{21}^{2}}{2 t_{21}}} e^{-\frac{x_{31}^{2}}{2 t_{31}}} .
$$

Here $\bar{\Phi}=\sqrt{\pi} \bar{\Psi}^{2}$ for the free-field theory; the normalisation condition is fixed by the twopoint function (4.9). This reproduces the free theory result.

Open Access. This article is distributed under the terms of the Creative Commons Attribution License (CC-BY 4.0), which permits any use, distribution and reproduction in any medium, provided the original author(s) and source are credited.

\section{References}

[1] K.G. Wilson and J.B. Kogut, The renormalization group and the $\epsilon$-expansion, Phys. Rept. 12 (1974) 75 [INSPIRE].

[2] R.J. Glauber, Time-Dependent Statistics of the Ising Model, J. Math. Phys. 4 (1963) 294.

[3] B.I. Halperin, P.C. Hohenberg and S.-k. Ma, Renormalization-group methods for critical dynamics: 1. Recursion relations and effects of energy conservation, Phys. Rev. B 10 (1974) 139 [inSPIRE].

[4] J.A. Hertz, Quantum critical phenomena, Phys. Rev. B 14 (1976) 1165 [InSPIRE].

[5] A.A. Migdal, A.M. Polyakov and K.A. Ter-Martirosian, Theory of Interacting Pomerons, Phys. Lett. B 48 (1974) 239 [inSPIRE].

[6] J.L. Cardy and R.L. Sugar, Directed Percolation and Reggeon Field Theory, J. Phys. A 13 (1980) L423 [INSPIRE].

[7] H. Hinrichsen, Critical phenomena in nonequilibrium systems, Adv. Phys. 49 (2000) 815 [cond-mat/0001070] [INSPIRE].

[8] Y. Pomeau, Front motion, metastability and subcritical bifurcations in hydrodynamics, Physica D 23 (1986) 3.

[9] M. Sano and K. Tamai, A Universal Transition to Turbulence in Channel Flow, Nature Phys. 12 (2016) 249 [arXiv:1510.07868].

[10] M. Kardar, G. Parisi and Y.-C. Zhang, Dynamic Scaling of Growing Interfaces, Phys. Rev. Lett. 56 (1986) 889 [INSPIRE].

[11] J. Cardy, Scaling and Renormalization in Statistical Physics, Cambridge Lecture Notes in Physics, Cambridge University Press, Cambridge, U.K. (1996).

[12] J. Cardy, Field Theory and Nonequilibrium Statistical Mechanics, Lectures Presented at the Troisieme Cycle de la Suisse Romande, (1999), https://www-thphys.physics.ox.ac.uk/people/JohnCardy/. 
[13] D.M. Eagles, Possible Pairing without Superconductivity at Low Carrier Concentrations in Bulk and Thin-Film Superconducting Semiconductors, Phys. Rev. 186 (1969) 456 [INSPIRE].

[14] A.J. Leggett, Diatomic molecules and cooper pairs, in Modern Trends in the Theory of Condensed Matter, vol. 115, pp. 13-27, Springer Berlin Heidelberg, Berlin, Heidelberg, Germany (1980).

[15] P. Nozières and S. Schmitt-Rink, Bose condensation in an attractive fermion gas: From weak to strong coupling superconduct ivity, J. Low Temp. Phys. 59 (1985) 195 [INSPIRE].

[16] T. Mehen, I.W. Stewart and M.B. Wise, Conformal invariance for nonrelativistic field theory, Phys. Lett. B 474 (2000) 145 [hep-th/9910025] [INSPIRE].

[17] S. Giorgini, L.P. Pitaevskii and S. Stringari, Theory of ultracold atomic Fermi gases, Rev. Mod. Phys. 80 (2008) 1215 [arXiv:0706.3360] [INSPIRE].

[18] K.G. Wilson, Nonlagrangian models of current algebra, Phys. Rev. 179 (1969) 1499 [INSPIRE].

[19] L.P. Kadanoff, Operator Algebra and the Determination of Critical Indices, Phys. Rev. Lett. 23 (1969) 1430 [inSPIRE].

[20] A.M. Polyakov, Conformal symmetry of critical fluctuations, JETP Lett. 12 (1970) 381 [Pisma Zh. Eksp. Teor. Fiz. 12 (1970) 538] [INSPIRE].

[21] A.M. Polyakov, Non-Hamiltonian approach to conformal quantum field theory, Sov. Phys. JETP 39 (1974) 10 [Zh. Eksp. Teor. Fiz. 66 (1974) 23].

[22] A.A. Belavin, A.M. Polyakov and A.B. Zamolodchikov, Infinite Conformal Symmetry in Two-Dimensional Quantum Field Theory, Nucl. Phys. B 241 (1984) 333 [inSPIRE].

[23] R. Rattazzi, V.S. Rychkov, E. Tonni and A. Vichi, Bounding scalar operator dimensions in 4D CFT, JHEP 12 (2008) 031 [arXiv:0807.0004] [INSPIRE].

[24] S. Rychkov, EPFL Lectures on Conformal Field Theory in D $\geq 3$ Dimensions, arXiv: 1601.05000 [INSPIRE].

[25] D. Poland, S. Rychkov and A. Vichi, The Conformal Bootstrap: Theory, Numerical Techniques, and Applications, Rev. Mod. Phys. 91 (2019) 015002 [arXiv:1805.04405] [INSPIRE].

[26] C.R. Hagen, Scale and conformal transformations in galilean-covariant field theory, Phys. Rev. D 5 (1972) 377 [INSPIRE].

[27] U. Niederer, The maximal kinematical invariance group of the free Schrödinger equation, Helv. Phys. Acta 45 (1972) 802 [inSPIRE].

[28] Y. Nakayama, Scale invariance vs conformal invariance, Phys. Rept. 569 (2015) 1 [arXiv:1302.0884] [INSPIRE].

[29] Y. Nakayama, Gravity Dual for Reggeon Field Theory and Non-linear Quantum Finance, Int. J. Mod. Phys. A 24 (2009) 6197 [arXiv:0906.4112] [InSPIRE].

[30] V.K. Dobrev, H.D. Doebner and C. Mrugalla, Lowest weight representations of the Schrödinger algebra and generalized heat/Schrödinger equations, Rept. Math. Phys. 39 (1997) 201.

[31] V.K. Dobrev, Non-Relativistic Holography - A Group-Theoretical Perspective, Int. J. Mod. Phys. A 29 (2014) 1430001 [arXiv:1312.0219] [INSPIRE]. 
[32] M. Henkel, Schrödinger invariance in strongly anisotropic critical systems, J. Statist. Phys. 75 (1994) 1023 [hep-th/9310081] [INSPIRE].

[33] M. Henkel and J. Unterberger, Schrödinger invariance and space-time symmetries, Nucl. Phys. B 660 (2003) 407 [hep-th/0302187] [INSPIRE].

[34] A.M. Polyakov, Turbulence without pressure, Phys. Rev. E 52 (1995) 6183 [hep-th/9506189] [INSPIRE].

[35] E. Braaten and L. Platter, Exact Relations for a Strongly Interacting Fermi Gas from the Operator Product Expansion, Phys. Rev. Lett. 100 (2008) 205301 [arXiv:0803.1125] [INSPIRE].

[36] K.G. Wilson, Operator product expansions and anomalous dimensions in the thirring model, Phys. Rev. D 2 (1970) 1473 [INSPIRE].

[37] L.P. Kadanoff, Correlations along a Line in the Two-Dimensional Ising Model, Phys. Rev. 188 (1969) 859.

[38] L.P. Kadanoff and H. Ceva, Determination of an opeator algebra for the two-dimensional Ising model, Phys. Rev. B 3 (1971) 3918 [InSPIRE].

[39] F. Calogero, Solution of a three-body problem in one-dimension, J. Math. Phys. 10 (1969) 2191 [INSPIRE].

[40] F. Calogero, Ground state of one-dimensional N body system, J. Math. Phys. 10 (1969) 2197 [INSPIRE].

[41] C. Marchioro, Solution of a three-body scattering problem in one dimension, J. Math. Phys. 11 (1970) 2193 [INSPIRE].

[42] F. Calogero, Solution of the one-dimensional $N$ body problems with quadratic and/or inversely quadratic pair potentials, J. Math. Phys. 12 (1971) 419 [INSPIRE].

[43] F. Calogero, Erratum: Solution of the one-dimensional N-body problems with quadratic and/or inversely quadratic pair potentials [J. Math. Phys. 12 (1971) 419], J. Math. Phys. 37 (1996) 3646.

[44] Y. Nishida and D.T. Son, Nonrelativistic conformal field theories, Phys. Rev. D 76 (2007) 086004 [arXiv: 0706.3746] [INSPIRE].

[45] W.D. Goldberger, Z.U. Khandker and S. Prabhu, OPE convergence in non-relativistic conformal field theories, JHEP 12 (2015) 048 [arXiv: 1412.8507] [INSPIRE].

[46] M. Lüscher, Operator product expansions on the vacuum in conformal quantum field theory in two spacetime dimensions, Commun. Math. Phys. 50 (1976) 23 [INSPIRE].

[47] G. Mack, Convergence of Operator Product Expansions on the Vacuum in Conformal Invariant Quantum Field Theory, Commun. Math. Phys. 53 (1977) 155 [inSPIRE].

[48] D. Pappadopulo, S. Rychkov, J. Espin and R. Rattazzi, OPE Convergence in Conformal Field Theory, Phys. Rev. D 86 (2012) 105043 [arXiv:1208.6449] [InSPIRE].

[49] K.G. Wilson and W. Zimmermann, Operator product expansions and composite field operators in the general framework of quantum field theory, Commun. Math. Phys. $\mathbf{2 4}$ (1972) 87 [INSPIRE].

[50] P.H. Ginsparg, Applied conformal field theory, hep-th/9108028 [INSPIRE]. 
[51] J.-C. Le Guillou and J. Zinn-Justin, Large-Order Behaviour of Perturbation Theory, North-Holland (1990).

[52] S. Golkar and D.T. Son, Operator Product Expansion and Conservation Laws in Non-Relativistic Conformal Field Theories, JHEP 12 (2014) 063 [arXiv:1408.3629] [INSPIRE].

[53] X. Bekaert, E. Meunier and S. Moroz, Symmetries and currents of the ideal and unitary Fermi gases, JHEP 02 (2012) 113 [arXiv:1111.3656] [INSPIRE].

[54] S. Pal, Unitarity and universality in nonrelativistic conformal field theory, Phys. Rev. D 97 (2018) 105031 [arXiv:1802.02262] [INSPIRE].

[55] B. Sutherland, Quantum many body problem in one-dimension: Ground state, J. Math. Phys. 12 (1971) 246 [INSPIRE].

[56] B. Sutherland, Quantum many body problem in one-dimension, J. Math. Phys. 12 (1971) 251 [INSPIRE].

[57] B. Sutherland, Exact results for a quantum many body problem in one-dimension, Phys. Rev. A 4 (1971) 2019 [inSPIRE].

[58] B. Sutherland, Exact results for a quantum many body problem in one-dimension. 2, Phys. Rev. A 5 (1972) 1372 [InSPIRE].

[59] L.D. Landau and E.M. Lifshitz, Quantum Mechanics: Non-Relativistic Theory, 3rd revised edition, Butterworth-Heinemann, (1977).

[60] A.P. Polychronakos, Physics and Mathematics of Calogero particles, J. Phys. A 39 (2006) 12793 [hep-th/0607033] [INSPIRE].

[61] A.P. Polychronakos, Nonrelativistic Bosonization and Fractional Statistics, Nucl. Phys. B 324 (1989) 597 [INSPIRE].

[62] G. Burdet and M. Perrin, Many-body realization of the Schrödinger algebra, Lett. Nuovo Cim. 4S2 (1972) 651 [INSPIRE].

[63] P.J. Gambardella, Exact results in quantum many-body systems of interacting particles in many dimensions with $\overline{\mathrm{SU}(1,1)}$ as the dynamical group, J. Math. Phys. 16 (1975) 1172.

[64] V. de Alfaro, S. Fubini and G. Furlan, Conformal invariance in quantum mechanics, Nuovo Cim. A 34 (1976) 569.

[65] A.I. Larkin and D.E. Khmel'nitskiu, Phase Transition in Uniaxial Ferroelectrics, Sov. Phys. JETP 29 (1969) 1123 [Zh. Eksp. Teor. Fiz. 56 (1969) 2087].

[66] E. Brézin and J. Zinn-Justin, Critical behavior of uniaxial systems with strong dipolar interactions, Phys. Rev. B 13 (1976) 251.

[67] M.E. Fisher, S.-k. Ma and B.G. Nickel, Critical Exponents for Long-Range Interactions, Phys. Rev. Lett. 29 (1972) 917 [INSPIRE].

[68] C. Behan, L. Rastelli, S. Rychkov and B. Zan, A scaling theory for the long-range to short-range crossover and an infrared duality, J. Phys. A 50 (2017) 354002 [arXiv: 1703.05325] [INSPIRE].

[69] C. Behan, L. Rastelli, S. Rychkov and B. Zan, Long-range critical exponents near the short-range crossover, Phys. Rev. Lett. 118 (2017) 241601 [arXiv:1703.03430] [INSPIRE]. 
[70] C. Behan, Bootstrapping the long-range Ising model in three dimensions, J. Phys. A 52 (2019) 075401 [arXiv:1810.07199] [InSPIRE].

[71] G.E. Astrakharchik, D.M. Gangardt, Y.E. Lozovik and I.A. Sorokin, Off-diagonal correlations of the Calogero-Sutherland model, Phys. Rev. E 74 (2006) 021105 [cond-mat/0512470].

[72] D. Peak and A. Inomata, Summation over Feynman Histories in Polar Coordinates, J. Math. Phys. 10 (1969) 1422.

[73] A. Volovich and C. Wen, Correlation Functions in Non-Relativistic Holography, JHEP 05 (2009) 087 [arXiv:0903.2455] [INSPIRE].

[74] H.M. Srivastava and P.W. Karlsson, Multiple Gaussian Hypergeometric Series, Ellis Horwood (1985).

[75] U. Niederer, The maximal kinematical invariance group of the harmonic oscillator, Helv. Phys. Acta 46 (1973) 191 [INSPIRE].

[76] F. Werner and Y. Castin, The unitary gas in an isotropic harmonic trap: Symmetry properties and applications, Phys. Rev. A 74 (2006) 053604 [cond-mat/0607821] [INSPIRE].

[77] L. Brink, T.H. Hansson and M.A. Vasiliev, Explicit solution to the $N$ body Calogero problem, Phys. Lett. B 286 (1992) 109 [hep-th/9206049] [INSPIRE].

[78] F.W.J. Olver et al., NIST Digital Library of Mathematical Functions, http://dlmf.nist.gov/, Release 1.0 .27 of $2020-06-15$.

[79] M.V. Barry, Uniform Asymptotic Smoothing of Stokes's Discontinuities, Proc. Roy. Soc. Lond. A 422 (1989) 7 [INSPIRE].

[80] R.B. Paris, A note on the asymptotics of the modified Bessel functions on the Stokes lines, Mathematica Eterna 7 (2017) 407 [arXiv:1708.09656].

[81] G.V. Dunne, Introduction to Resurgence, Trans-series and Non-perturbative Physics, (2018), Lectures at Nonperturbative and Numerical Approaches to Quantum Gravity, String Theory and Holography program at ICTS Bangalore, https://www.icts.res.in/program/NUMSTRINGS2018/talks.

[82] C.A. Fuertes and S. Moroz, Correlation functions in the non-relativistic AdS/CFT correspondence, Phys. Rev. D 79 (2009) 106004 [arXiv:0903.1844] [INSPIRE].

[83] Y. Sekino and Y. Nishida, Field-theoretical aspects of one-dimensional Bose and Fermi gases with contact interactions, Phys. Rev. A 103 (2021) 043307 [arXiv:2011.11971] [INSPIRE].

[84] M. Henkel, From dynamical scaling to local scale-invariance: a tutorial, Eur. Phys. J. ST 226 (2017) 605 [arXiv:1610.06122] [INSPIRE].

[85] J. Teschner, On the Liouville three point function, Phys. Lett. B 363 (1995) 65 [hep-th/9507109] [INSPIRE].

[86] D.J. Amit, Y.Y. Goldschmidt and G. Grinstein, Renormalization Group Analysis of the Phase Transition in the 2D Coulomb Gas, sine-Gordon Theory and xy Model, J. Phys. A 13 (1980) 585 [INSPIRE].

[87] V.E. Korepin, Above-barrier reflection of solitons, Theor. Math. Phys. 34 (1978) 1.

[88] A.B. Zamolodchikov and A.B. Zamolodchikov, Factorized s Matrices in Two-Dimensions as the Exact Solutions of Certain Relativistic Quantum Field Models, Annals Phys. 120 (1979) 253 [INSPIRE]. 
[89] A. Kapustin and S. Skorik, On the nonrelativistic limit of the quantum sine-Gordon model with integrable boundary condition, Phys. Lett. A 196 (1994) 47 [hep-th/9409097] [INSPIRE].

[90] P. Di Francesco, P. Mathieu and D. Sénéchal, Conformal Field Theory, Graduate Texts in Contemporary Physics, Springer, New York, U.S.A. (1997).

[91] A. Luther and I. Peschel, Calculation of critical exponents in two-dimensions from quantum field theory in one-dimension, Phys. Rev. B 12 (1975) 3908 [INSPIRE].

[92] M. Bander and C. Itzykson, Quantum Field Theory Calculation of Two-Dimensional Ising Model Correlation Function, Phys. Rev. D 15 (1977) 463 [InSPIRE].

[93] J.B. Zuber and C. Itzykson, Quantum Field Theory and the Two-Dimensional Ising Model, Phys. Rev. D 15 (1977) 2875 [inSPIRE].

[94] B. Schroer and T.T. Truong, Direct Construction of the Quantum Field Operators of the $D=2$ Ising Model, Phys. Lett. B 73 (1978) 149 [inSPIRE].

[95] B. Schroer and T.T. Truong, The Order/Disorder Quantum Field Operators Associated to the Two-dimensional Ising Model in the Continuum Limit, Nucl. Phys. B 144 (1978) 80 [INSPIRE].

[96] B. Schroer and T.T. Truong, The relativistic quantum fields of the D=2 Ising model, Phys. Lett. B 72 (1978) 371 [inSPIRE].

[97] L.P. Kadanoff and A.C. Brown, Correlation functions on the critical lines of the Baxter and Ashkin-Teller models, Annals Phys. 121 (1979) 318 [INSPIRE].

[98] P. Di Francesco, H. Saleur and J.B. Zuber, Critical Ising Correlation Functions in the Plane and on the Torus, Nucl. Phys. B 290 (1987) 527 [INSPIRE].

[99] D. Boyanovsky, Field theory of the two-dimensional Ising model: Conformal invariance, order and disorder, and bosonization, Phys. Rev. B 39 (1989) 6744.

[100] B.U. Felderhof, Note on spin relaxation of the ising chain, Rept. Math. Phys. 2 (1971) 151.

[101] T.D. Schultz, D.C. Mattis and E.H. Lieb, Two-dimensional Ising model as a soluble problem of many fermions, Rev. Mod. Phys. 36 (1964) 856 [InSPIRE].

[102] C. Godrèche and J.M. Luck, Response of non-equilibrium systems at criticality: Exact results for the Glauber-Ising chain, J. Phys. A 33 (2000) 1151 [cond-mat/9911348].

[103] J. Wolfes, On a one-dimensional four-body scattering system, Annals Phys. 85 (1974) 454 [INSPIRE].

[104] F. Calogero and C. Marchioro, Exact solution of a one-dimensional three-body scattering problem with two-body and/or three-body inverse-square potentials, J. Math. Phys. 15 (1974) 1425 [INSPIRE].

[105] M.A. Olshanetsky and A.M. Perelomov, Classical integrable finite dimensional systems related to Lie algebras, Phys. Rept. 71 (1981) 313 [INSPIRE].

[106] M.A. Olshanetsky and A.M. Perelomov, Quantum Integrable Systems Related to Lie Algebras, Phys. Rept. 94 (1983) 313 [INSPIRE].

[107] G.W. Semenoff, Dilaton in a cold Fermi gas, arXiv:1808.03861 [INSPIRE].

[108] N. Kawakami and S.-K. Yang, Finite size scaling in one-dimensional quantum liquid with long range interaction, Phys. Rev. Lett. 67 (1991) 2493 [INSPIRE]. 
[109] N. Kawakami and S.-K. Yang, Application of conformal field theory to electron systems, Prog. Theor. Phys. Suppl. 106 (1991) 157 [INSPIRE].

[110] S. Iso, Long distance universality of Laughlin state and Calogero-Sutherland model, Mod. Phys. Lett. A 9 (1994) 2123 [cond-mat/9404075] [INSPIRE].

[111] S. Iso and S.J. Rey, Collective field theory of the fractional quantum hall edge state and the Calogero-Sutherland model, Phys. Lett. B 352 (1995) 111 [hep-th/9406192] [InSPIRE].

[112] S. Iso, Anyon basis of $c=1$ conformal field theory, Nucl. Phys. B 443 (1995) 581 [hep-th/9411051] [INSPIRE].

[113] R. Caracciolo, A. Lerda and G.R. Zemba, The W (1+infinity) effective theory of the Calogero-Sutherland model and Luttinger systems, Phys. Lett. B 352 (1995) 304 [hep-th/9503229] [INSPIRE].

[114] J.M. Leinaas and J. Myrheim, Intermediate statistics for vortices in superfluid films, Phys. Rev. B 37 (1988) 9286 [INSPIRE].

[115] A.P. Polychronakos, Exact anyonic states for a general quadratic Hamiltonian, Phys. Lett. B 264 (1991) 362 [INSPIRE].

[116] G.V. Dunne, A. Lerda, S. Sciuto and C.A. Trugenberger, Exact multi-anyon wave functions in a magnetic field, Nucl. Phys. B 370 (1992) 601 [INSPIRE].

[117] T.H. Hansson, J.M. Leinaas and J. Myrheim, Dimensional reduction in anyon systems, Nucl. Phys. B 384 (1992) 559 [InSPIRE].

[118] L. Brink, T.H. Hansson, S. Konstein and M.A. Vasiliev, The Calogero model: Anyonic representation, fermionic extension and supersymmetry, Nucl. Phys. B 401 (1993) 591 [hep-th/9302023] [INSPIRE].

[119] H. Azuma and S. Iso, Explicit relation of quantum hall effect and Calogero-Sutherland model, Phys. Lett. B 331 (1994) 107 [hep-th/9312001] [INSPIRE].

[120] A.P. Polychronakos, Quantum Hall states as matrix Chern-Simons theory, JHEP 04 (2001) 011 [hep-th/0103013] [INSPIRE].

[121] S. Ouvry and A.P. Polychronakos, Mapping the Calogero model on the Anyon model, Nucl. Phys. B 936 (2018) 189 [arXiv:1805.09899] [INSPIRE]. 